\title{
IRREDUCIBLE REPRESENTATIONS OF THE FREE PRODUCT OF GROUPS
}

\author{
WALDEMAR HEBISCH AND WOJCIECH MEOTKOWSKI
}

\begin{abstract}
We study some properties, such as uniform boundedness, unitarity and irreducibility, of a class of representations of the free product of groups. In particular we show that the spherical functions on the free product of two groups, introduced by Cartwright and Soardi, are coefficients of irreducible representations.
\end{abstract}

\section{INTRODUCTION}

There have been several attempts to construct representations of free product of groups or algebras. One family of such constructions is related to the commutative convolution algebra of radial functions on the free group $*_{i \in I} \mathbb{Z}$, see $[\mathrm{FP} 1, \mathrm{FP} 2, \mathrm{~T} 2, \mathrm{~K}$, $\mathrm{KS} 1, \mathrm{MZ}, \mathrm{PS}]$, or on the free product of cyclic groups of the same order, see [IP, Wy]. On the other hand there are developed methods to produce a representation of $G=*_{i \in I} G_{i}$ (or, more generally, of a unital free product $\mathcal{A}=*_{i \in I} \mathcal{A}_{i}$ of $*$-algebras $\mathcal{A}_{i}$ ) from those of $G_{i}$ 's (or $\mathcal{A}_{i}$ 's), see [B1, Av, Vo, VDN, BS, BLS, M2, M3, M4, M5].

Much effort has been devoted to study irreducibility of such representations. For those related to radial functions the method is to study the projection on a cyclic vector, see [FP1, FP2, PS, MZ, IP, Sz]. Interesting constructions of irreducible representations of the free group are due to Kuhn, Steger [KS3, KS4] and Paschke [P1, P2]. Let us also mention papers by Młotkowski [M2], Kuhn and Steger [KS2]. The former proves irreducibility for a family of representations on the free product of infinite groups, the latter proves that for a family of representations of the infinitely generated free group.

The starting point of this paper is the observation that for the free product group $G=*_{i \in I} G_{i}$ there is an associated unital, noncommutative (unless $|I|=1$ ) *-algebra $\mathcal{A}(\tau)$ depending only on the parameters $\tau_{i}:=\left(\left|G_{i}\right|-1\right)^{-1}$ (cf. [M1]). If all $G_{i}$ 's are finite then $\mathcal{A}(\tau)$ can be identified with the convolution algebra of finitely supported type dependent functions on $G$. For a representation $\pi_{0}$ of $\mathcal{A}(\tau)$, acting on a Hilbert space, we construct in Section 5 a representation $\pi$ of $G$, acting on a larger Hilbert space. We say that $\pi$ is induced from $\pi_{0}$. We prove that $\pi$ inherits many properties of $\pi_{0}$, namely:

1. If $\pi_{0}$ is a $*$-representation then $\pi$ is unitary (Theorem 5.5.iv).

2. If $\pi_{0}$ satisfies some version of uniform boundedness then $\pi$ is uniformly bounded (Theorem 5.5.v).

3. If $\pi_{0}$ and $\sigma_{0}$ are representations of $\mathcal{A}(\tau)$ which are disjoint from the regular representation of $\mathcal{A}(\tau)$ and if $\pi_{0}$ and $\sigma_{0}$ are not equivalent (resp. $\pi_{0}$ and $\sigma_{0}$ are disjoint) then so are the induced representations (Theorem 5.10).

4. If a $*$-representation $\pi_{0}$ of $\mathcal{A}(\tau)$ is weakly contained in a $*$-representation $\sigma_{0}$ then the same holds for the induced unitary representations (Theorem 5.11). In particular, if $\pi_{0}$ is weakly contained in the regular representation of $\mathcal{A}(\tau)$ (defined in Section 2)

2000 Mathematics Subject Classification. Primary 43A65; Secondary 46L09, 20 E06.

Key words and phrases. free product, irreducible representation. 
then the induced representation is weakly contained in the regular representation of $G$. In Section 3 we adapt Haagerup's [Ha] method to characterize those representations of $\mathcal{A}(\tau)$ which are weakly contained in the regular representation.

5. Finally we prove that if $\pi_{0}$ is a $*$-representation of $\mathcal{A}(\tau)$ which is irreducible then so is $\pi$, unless $\pi_{0}$ is contained in the regular representation of $\mathcal{A}(\tau)$ (Theorem 6.4). We will see in Section 2 that there are at most two irreducible subrepresentations of the regular representations of $\mathcal{A}(\tau)$, and they are one-dimensional.

In the nonunitary case, we have managed to prove that if $\pi_{0}$ is a finitely dimensional irreducible representation of $\mathcal{A}(\tau)$, not equivalent to a $*$-representation, then $\pi$ is fully irreducible (Theorem 6.8).

In the last section we apply our results to the free product of two groups $G=G_{+} * G_{-}$, with $\left|G_{+}\right|=r,\left|G_{-}\right|=s, r>s \geq 2$ (an important example is $\mathbb{Z}_{3} * \mathbb{Z}_{2} \cong \operatorname{PSL}(2, \mathbb{Z})$ ). Cartwright and Soardi [CS] studied a family of spherical functions $\phi_{\lambda}$ on such group, with $\lambda \in \mathbb{C}, \lambda \neq(r+s-4) / 2$. Here we prove that every $\phi_{\lambda}$ is a coefficient of a fully irreducible (unless $\lambda=-2$ or $\lambda=s-2$ ) representation of $G$. Moreover, this representation is unitary if and only if $\lambda \in[-2, s-2] \cup[r-2, r+s-2]$.

Acknowledgments. W. Hebisch is supported by MNiSW: 5 P03A 050 29, and by ToK: MTKD-CT-2004-013389. W. Młotkowski is supported by MNiSW: 1 P03A 013 30, by ToK: MTKD-CT-2004-013389, by 7010 POLONIUM project: "Non-Commutative Harmonic Analysis with Applications to Operator Spaces, Operator Algebras and Probability", and by joint PAN-JSPS project: "Noncommutative harmonic analysis on discrete structures with applications to quantum probability".

We would like to express our deep gratitude to Tim Steger for valuable discussions on the results of this paper.

\section{The Algebra $\mathcal{A}(\tau)$}

If $X$ is a set then $\mathcal{F}(X)\left(\right.$ resp. $\left.\mathcal{F}_{0}(X)\right)$ will stand for the class of finitely supported complex functions on $X$ (which satisfy $\sum_{x \in X} f(x)=0$ ). For two complex functions $f, g$ on $X$ we put $\langle f, g\rangle:=\sum_{x \in X} f(x) g(x)$ whenever the sum is well defined.

We recall some notions which were studied in [M1]. Let $I$ be a nonempty fixed set and let $S(I)$ denote the set of all formal words of the form

$$
u=i_{1} i_{2} \ldots i_{m}, \quad \text { with } m \geq 0, i_{k} \in I \text { and } i_{k} \neq i_{k+1} \text { for all } k<m,
$$

including the empty word $e$. The length $m$ will be denoted by $|u|$. For $i \in I$ we define

$$
S_{i}:=\left\{i_{1} i_{2} \ldots i_{m} \in S(I): m=0 \text { or } i_{1} \neq i\right\} .
$$

Let $\tau$ be a function $I \rightarrow[0,+\infty)$. We extend $\tau$ to $S(I)$ by putting

$$
\tau(u):=\tau_{i_{1}} \tau_{i_{2}} \ldots \tau_{i_{m}}
$$

for $u$ as in (1.1), in particular $\tau(e)=1$. Then we define $\tau$-convolution as an associative operation on $\mathcal{F}(S(I))$, such that $\delta_{e}$ is the unit and for each $u$ as in (1.1), with $|u| \geq 1$,

$$
\delta_{i} *_{\tau} \delta_{u}= \begin{cases}\left(1-\tau_{i}\right) \delta_{u}+\tau_{i} \delta_{u^{\prime}} & \text { if } i=i_{1}, \\ \delta_{i u} & \text { if } i \neq i_{1},\end{cases}
$$

where $u^{\prime}=i_{2} \ldots i_{m}$ and $i u=i i_{1} \ldots i_{m}$ ( $\delta_{x}$ denotes the characteristic function of the point set $\{x\})$. We also define involution by $f^{*}(u):=\overline{f\left(u^{*}\right)}$, where $u^{*}:=i_{m} \ldots i_{2} i_{1}$ for $u$ as in (1.1). In this way $\mathcal{F}(S(I))$ becomes a unital $*$-algebra, which we will denote 
by $\mathcal{A}(\tau)$. Note that $\mathcal{A}(\tau)$ can be defined as the unital free $*$-algebra on $I$, subject to the relations $i^{2}=\left(1-\tau_{i}\right) i+\tau_{i} e$ and $i^{*}=i$ for $i \in I$, and is also an example of generic algebra (see $[\mathrm{Hu}])$.

Observe that the characters of $\mathcal{A}(\tau)$, i.e. functions $\phi$ on $S(I)$ such that $\left\langle\phi, f *_{\tau} g\right\rangle=$ $\langle\phi, f\rangle\langle\phi, g\rangle$ holds for all $f, g \in \mathcal{F}(S(I))$, are of the form $\phi(u)=\phi\left(i_{1}\right) \ldots \phi\left(i_{m}\right)$ for $u$ as in (1.1), where $\phi(i) \in\left\{1,-\tau_{i}\right\}$. In particular, taking $\phi \equiv 1$ we see that $\mathcal{F}_{0}(S(I))=\operatorname{ker} \phi$ is an ideal in $\mathcal{A}(\tau)$.

For two functions $\sigma, \tau: I \rightarrow[0,+\infty)$ we define a $*$-isomorphism $H_{\tau \sigma}: \mathcal{A}(\sigma) \rightarrow \mathcal{A}(\tau)$ putting

$$
H_{\tau \sigma}\left(\delta_{i}\right):=\frac{1+\sigma_{i}}{1+\tau_{i}} \delta_{i}+\frac{\tau_{i}-\sigma_{i}}{1+\tau_{i}} \delta_{e}
$$

for each $i \in I$. Indeed, one can check that

$$
H_{\tau \sigma}\left(\delta_{i}\right) *_{\tau} H_{\tau \sigma}\left(\delta_{i}\right)=\left(1-\sigma_{i}\right) H_{\tau \sigma}\left(\delta_{i}\right)+\sigma_{i} \delta_{e} .
$$

Observe also that for $f, g \in \mathcal{F}(S(I))$

$$
\left(f *_{\tau} g^{*}\right)(e)=\left(g^{*} *_{\tau} f\right)(e)=\sum_{u \in S(I)} f(u) \overline{g(u)} \tau(u) .
$$

Definition 1.1. A function $\phi: S(I) \rightarrow \mathbf{C}$ is said to be $\tau$-positive definite (resp. $\tau$ negative definite) if $\left\langle\phi, f^{*} *_{\tau} f\right\rangle \geq 0$ (resp. $\left\langle\phi, f_{0}^{*} *_{\tau} f_{0}\right\rangle \leq 0$ ) holds for every $f \in \mathcal{F}(S(I))$ (resp. for every $f_{0} \in \mathcal{F}_{0}(S(I))$ ). We will denote by $\mathcal{P}(\tau)$ and $\mathcal{N}(\tau)$ the class of $\tau$ positive and $\tau$-negative definite functions on $S(I)$ respectively, and by $B(\tau)$ the class of linear combinations of $\tau$-positive definite functions on $S(I)$, i.e. the $\tau$-Fourier-Stielties algebra.

In particular, the characters of $\mathcal{A}(\tau)$ are $\tau$-positive definite.

Proposition 1.2. Suppose that for every $i \in I$ we have $0 \leq \sigma_{i} \leq \tau_{i}$. Then

$$
\mathcal{P}(\sigma) \subseteq \mathcal{P}(\tau) \quad \text { and } \quad \mathcal{N}(\sigma) \subseteq \mathcal{N}(\tau) .
$$

Proof. It is sufficient to show that for every $f \in \mathcal{F}(S(I))$ we have

$$
f^{*} *_{\tau} f=f^{*} *_{\sigma} f+R,
$$

where $R$ is a finite sum of terms of the form $f_{0}^{*} *_{\sigma} f_{0}$, with $f_{0} \in \mathcal{F}_{0}(S(I))$. We proceed by induction on $n$, the maximal length of words in the support of $f$. Decompose $f$ as

$$
f=f(e) \delta_{e}+\sum_{i \in I} \delta_{i} * f_{i}
$$

(we write simply " $*$ " whenever "* $*_{\tau}$ " can be replaced by "**") with $\operatorname{supp} f_{i} \subseteq S_{i}$, see (1.2). Then, by induction, we can write $f_{i}^{*} *_{\tau} f_{i}=f_{i}^{*} *_{\sigma} f_{i}+R(i)$. Now we have

$$
f^{*} *_{\tau} f-f^{*} *_{\sigma} f=\sum_{i \in I}\left\{\left(\delta_{i} * f_{i}\right)^{*} *_{\tau}\left(\delta_{i} * f_{i}\right)-\left(\delta_{i} * f_{i}\right)^{*} *_{\sigma}\left(\delta_{i} * f_{i}\right)\right\}
$$

and

$$
\begin{aligned}
\left(\delta_{i} * f_{i}\right)^{*} *_{\tau} & \left(\delta_{i} * f_{i}\right)=\left(1-\tau_{i}\right) f_{i}^{*} * \delta_{i} * f_{i}+\tau_{i} f_{i}^{*} *_{\tau} f_{i} \\
& =f_{i}^{*} *_{\sigma}\left(\left(1-\tau_{i}\right) \delta_{i}+\tau_{i} \delta_{e}\right) *_{\sigma} f_{i}+\tau_{i} R(i) \\
& =\left(\delta_{i} * f_{i}\right)^{*} *_{\sigma}\left(\delta_{i} * f_{i}\right)+\left(\tau_{i}-\sigma_{i}\right) f_{i}^{*} *_{\sigma}\left(\delta_{e}-\delta_{i}\right) *_{\sigma} f_{i}+\tau_{i} R(i) .
\end{aligned}
$$


To conclude we note that

$$
\delta_{e}-\delta_{i}=\frac{1}{1+\sigma_{i}}\left(\delta_{e}-\delta_{i}\right) *_{\sigma}\left(\delta_{e}-\delta_{i}\right) .
$$

We will identify, by $\phi(u):=\phi\left(\delta_{u}\right)$, the dual space $\mathcal{F}(S(I))^{\prime}$ with the space of complex functions on $S(I)$. Denote by $T_{\sigma \tau}$ the dual map to $H_{\tau \sigma}$, i.e. $T_{\sigma \tau}(\phi)=\phi \circ H_{\tau \sigma}$.

Proposition 1.3. $T_{\sigma \tau}$ maps $\mathcal{P}(\tau)$ onto $\mathcal{P}(\sigma), \mathcal{N}(\tau)$ onto $\mathcal{N}(\sigma)$ and $B(\tau)$ onto $B(\sigma)$.

Proof. For a $\tau$-positive definite function $\psi$ and for $f \in \mathcal{F}(S(I))$ we have

$$
\left\langle T_{\sigma \tau} \psi, f^{*} *_{\sigma} f\right\rangle=\left\langle\psi, H_{\tau \sigma}\left(f *_{\sigma} f\right)\right\rangle=\left\langle\psi, H_{\tau \sigma}(f)^{*} *_{\tau} H_{\tau \sigma}(f)\right\rangle \geq 0 .
$$

Proposition 1.4. Assume that $\left\{P_{i}\right\}_{i \in I}$ is a family of projections on a Hilbert space $\mathcal{H}_{0}, \zeta, \eta \in \mathcal{H}_{0}$ and let $A_{i}=\left(1+\tau_{i}\right) P_{i}-\tau_{i} \mathrm{Id}, B_{i}=\left(1+\sigma_{i}\right) P_{i}-\sigma_{i}$ Id. Define $\phi(u)=$ $\left[A_{i_{1}} A_{i_{2}} \ldots A_{i_{m}} \zeta, \eta\right]$ and $\psi(u)=\left[B_{i_{1}} B_{i_{2}} \ldots B_{i_{m}} \zeta, \eta\right]$ for $u=i_{1} i_{2} \ldots i_{m} \in S(I)$. Then $\psi=T_{\sigma \tau} \phi$.

Proof. Let $A: \mathcal{A}(\tau) \rightarrow \mathcal{B}\left(\mathcal{H}_{0}\right)$ and $B: \mathcal{A}(\sigma) \rightarrow \mathcal{B}\left(\mathcal{H}_{0}\right)$ be the unique homomorphisms which satisfy $A\left(\delta_{i}\right)=A_{i}$ and $B\left(\delta_{i}\right)=B_{i}$. Since

$$
B_{i}=\frac{1+\sigma_{i}}{1+\tau_{i}} A_{i}+\frac{\tau_{i}-\sigma_{i}}{1+\tau_{i}} \mathrm{Id},
$$

we have $B=A \circ H_{\tau \sigma}$. Now define $\Phi: \mathcal{B}\left(\mathcal{H}_{0}\right) \rightarrow \mathbb{C}$ putting $\Phi(T):=[T \zeta, \eta]$. Then

$$
\psi=\Phi \circ B=\Phi \circ A \circ H_{\tau \sigma}=\phi \circ H_{\tau \sigma}=T_{\sigma \tau}(\phi) .
$$

Now we prove that $\tau$-positive definiteness admits the standard GNS construction:

Proposition 1.5. Let $\phi$ be a complex function on $S(I)$ and let $\tau$ be a function $I \rightarrow$ $[0,+\infty)$. Then $\phi$ is $\tau$-positive definite (resp. belongs to $B(\tau)$ ) if and only if there exists a Hilbert space $\mathcal{H}_{0}$, a vector $\xi \in \mathcal{H}_{0}$ (resp. vectors $\zeta, \eta \in \mathcal{H}_{0}$ ) and a family $\left\{P_{i}\right\}_{i \in I}$ of orthogonal projections on $\mathcal{H}_{0}$ that for every $u=i_{1} i_{2} \ldots i_{m} \in S(I)$

$$
\phi(u)=\left[A_{i_{1}} A_{i_{2}} \ldots A_{i_{m}} \xi, \xi\right] \quad\left(\text { resp. } \quad \phi(u)=\left[A_{i_{1}} A_{i_{2}} \ldots A_{i_{m}} \zeta, \eta\right]\right),
$$

where $A_{i}=\left(1+\tau_{i}\right) P_{i}-\tau_{i} \mathrm{Id}$.

Proof. First assume that $\tau \equiv 1$. Then $\tau$-positive definiteness coincides with the usual one on the free product group $*_{i \in I} \mathbb{Z}_{2}$ and our assertion is well known in this case. The general case follows from Proposition 1.3 and 1.4.

It turns out that one part of the last proposition can be generalized.

Lemma 1.6. Assume that $\left\{A_{i}\right\}_{i \in I}$ is a family of operators on a Hilbert space $\mathcal{H}_{0}$ such that $-\tau_{i} \operatorname{Id} \leq A_{i} \leq \operatorname{Id}$ for every $i \in I$. Then for $\xi \in \mathcal{H}_{0}$ the function

$$
\phi\left(i_{1} \ldots i_{m}\right):=\left[A_{i_{1}} \ldots A_{i_{m}} \xi, \xi\right]
$$

is $\tau$-positive definite on $S(I)$.

Proof. Define a linear function $\Phi: \mathcal{F}(S(I)) \rightarrow \mathcal{B}\left(\mathcal{H}_{0}\right)$ by putting $\Phi\left(\delta_{u}\right):=\Phi(u):=$ $A_{i_{1}} \ldots A_{i_{m}}$ for $u=i_{1} \ldots i_{m}$ as in (1.1). We will prove that for every finitely supported function $\eta: S(I) \rightarrow \mathcal{H}_{0}$ we have

$$
\sum_{u, v \in S(I)}\left[\Phi\left(\delta_{v^{*}} *_{\tau} \delta_{u}\right) \eta(u), \eta(v)\right] \geq\left\|\sum_{u \in S(I)} \Phi(u) \eta(u)\right\|^{2} .
$$


We proceed by induction on $n:=\max \{|u|: u \in \operatorname{supp}(\eta)\}$. For $i \in I$ and $u=i_{1} \ldots i_{m} \in$ $S(I)$ define

$$
\eta_{i}(u):= \begin{cases}\eta(i u) & \text { if } u \in S_{i} \\ 0 & \text { otherwise }\end{cases}
$$

Then, by induction,

$$
R(i):=\sum_{u, v \in S(I)}\left[\Phi\left(\delta_{v^{*}} *_{\tau} \delta_{u}\right) \eta_{i}(u), \eta_{i}(v)\right]-\left\|\sum_{u \in S(I)} \Phi(u) \eta_{i}(u)\right\|^{2} \geq 0 .
$$

Note that $\Phi\left(v^{*} u\right)=\Phi^{*}(v) \Phi(u)$ holds if $u=e$ or $v=e$ or if $|u|,|v| \geq 1$ and $u, v$ start with different letters. Therefore

$$
\begin{aligned}
& \sum_{u, v \in S(I)}\left[\Phi\left(\delta_{v^{*}} *_{\tau} \delta_{u}\right) \eta(u), \eta(v)\right]-\sum_{u, v \in S(I)}[\Phi(u) \eta(u), \Phi(v) \eta(v)] \\
= & \sum_{i \in I} \sum_{u, v \in S_{i}}\left\{\left[\Phi\left(\delta_{v^{*}} *_{\tau}\left(\delta_{i} *_{\tau} \delta_{i}\right) *_{\tau} \delta_{u}\right) \eta(i u), \eta(i v)\right]-\left[A_{i}^{2} \Phi(u) \eta(i u), \Phi(v) \eta(i v)\right]\right\} \\
= & \sum_{i \in I} \sum_{u, v \in S_{i}}\left\{\left[\Phi\left(\left(1-\tau_{i}\right) \delta_{v^{*} i u}+\tau_{i} \delta_{v^{*}} *_{\tau} \delta_{u}\right) \eta_{i}(u), \eta_{i}(v)\right]-\left[A_{i}^{2} \Phi(u) \eta_{i}(u), \Phi(v) \eta_{i}(v)\right]\right\} \\
= & \sum_{i \in I}\left\{\tau_{i} R(i)+\left[\left(\left(1-\tau_{i}\right) A_{i}-A_{i}^{2}+\tau_{i} \operatorname{Id}\right) \xi_{i}, \xi_{i}\right]\right\} \geq 0,
\end{aligned}
$$

where $\xi_{i}:=\sum_{u \in S(I)} \Phi(u) \eta_{i}(u)$, because

$$
\left(1-\tau_{i}\right) A_{i}-A_{i}^{2}+\tau_{i} \mathrm{Id}=\left(\mathrm{Id}-A_{i}\right)\left(\tau_{i} \mathrm{Id}+A_{i}\right)
$$

is a nonnegative operator. Now, for $f \in \mathcal{F}(S(I))$ we have

$$
\left\langle\phi, f^{*} *_{\tau} f\right\rangle=\left[\Phi\left(f^{*} *_{\tau} f\right) \xi, \xi\right]=\sum_{u, v \in S(I)}\left[\left\langle\Phi, \delta_{v^{*}} *_{\tau} \delta_{u}\right\rangle f(u) \xi, f(v) \xi\right] \geq 0 .
$$

Taking $\mathcal{H}_{0}=\mathbb{C}$ we obtain a family of $\tau$-positive definite functions:

Corollary 1.7. Assume that $0 \leq r \leq 1$. Then the function $u \mapsto r^{|u|}$ is $\tau$-positive definite on $S(I)$ for every $\tau: I \rightarrow[0, \infty)$.

Now we can prove a version of the Schur theorem:

Corollary 1.8. Assume that $\phi_{1}$ and $\phi_{2}$ is $\tau^{(1)}$ - and $\tau^{(2)}$-positive definite on $S(I)$, respectively, where the functions $\tau^{(1)}, \tau^{(2)}: I \rightarrow[0, \infty)$ are such that $\tau_{i}^{(1)} \cdot \tau_{i}^{(2)} \leq 1$ for every $i \in I$. Then the product $\phi_{1} \cdot \phi_{2}$ is $\sigma$-positive definite, where $\sigma_{i}:=\max \left\{\tau_{i}^{(1)}, \tau_{i}^{(2)}\right\}$.

In particular, if $\tau: I \rightarrow[0,1]$ then $\mathcal{P}(\tau)$ is closed under pointwise multiplication.

Proof. For $k=1,2$, let $\left(\mathcal{H}_{k}, \pi_{k}, \xi_{k}\right)$ be the GNS triple for $\phi_{k}$, and let

$$
A_{k}(i):=\pi_{k}\left(\delta_{i}\right)=\left(1+\tau_{i}^{(k)}\right) P_{k}(i)-\tau_{i}^{(k)} \operatorname{Id}_{k}
$$

for a selfadjoint projection $P_{k}(i)$ on $\mathcal{H}_{k}$. Defining the operator $A(i):=A_{1}(i) \otimes A_{2}(i)$ on $\mathcal{H}_{1} \otimes \mathcal{H}_{2}$ we have

$$
\begin{gathered}
A(i)=P_{1}(i) \otimes P_{2}(i)-\tau_{i}^{(2)} P_{1}(i) \otimes\left(\operatorname{Id}_{2}-P_{2}(i)\right)-\tau_{i}^{(1)}\left(\operatorname{Id}_{1}-P_{1}(i)\right) \otimes P_{2}(i) \\
+\tau_{i}^{(1)} \cdot \tau_{i}^{(2)}\left(\operatorname{Id}_{1}-P_{1}(i)\right) \otimes\left(\mathrm{Id}_{2}-P_{2}(i)\right),
\end{gathered}
$$


so that $\mathcal{H}_{1} \otimes \mathcal{H}_{2}$ is decomposed into orthogonal direct sum of four subspaces and $A(i)$ acts on each of them by multiplying by $1,-\tau_{i}^{(2)},-\tau_{i}^{(1)}$ and $\tau_{i}^{(1)} \cdot \tau_{i}^{(2)}$ respectively, so that $-\sigma_{i} \mathrm{Id}_{1} \otimes \mathrm{Id}_{2} \leq A(i) \leq \mathrm{Id}_{1} \otimes \mathrm{Id}_{2}$. We also have

$$
\phi_{1}(u) \phi_{2}(u)=\left[A\left(i_{1}\right) \ldots A\left(i_{m}\right) \xi_{1} \otimes \xi_{2}, \xi_{1} \otimes \xi_{2}\right]
$$

for $u=i_{1} \ldots i_{m}$, which, by Lemma 1.6, concludes the proof.

\section{The Regular Representation of $\mathcal{A}(\tau)$}

Let us fix $\tau: I \rightarrow[0, \infty)$ and denote $I^{\text {fin }}:=\left\{i \in I: \tau_{i}>0\right\}$ (this notation will be justified in Section 6). Let $\mathcal{F}^{\text {fin }}(S(I))$ denote the class of those $f \in \mathcal{F}(S(I)$ for which $\operatorname{supp} f \subseteq S\left(I^{\text {fin }}\right)$. We will work on the Hilbert space $\ell^{2}(\tau)$ of complex functions $f$ on $S(I)$, with support in $S\left(I^{\text {fin }}\right)$, satisfying

$$
\|f\|_{2}^{2}:=\sum_{u \in S(I)}|f(u)|^{2} \tau(u)<\infty
$$

where $\tau(u)$ was defined in (1.3), with the scalar product

$$
[f, g]:=\sum_{u \in S(I)} f(u) \overline{g(u)} \tau(u) .
$$

According to (1.5), we have

$$
[f, g]=\left(f *_{\tau} g^{*}\right)(e)=\left(g^{*} *_{\tau} f\right)(e)
$$

for all $f, g \in \mathcal{F}^{\text {fin }}(S(I))$ and in view of Lemma 2.2 it remains true for all $f, g \in \ell^{2}(\tau)$.

There are two natural $*$-representations of $\mathcal{A}(\tau)$ acting on $\ell^{2}(\tau)$, namely the left and the right regular one:

$$
\lambda_{0}(a) f:=\left(a *_{\tau} f\right) \cdot \chi, \quad \rho_{0}(b) f:=\left(f *_{\tau} b^{*}\right) \cdot \chi
$$

for $a, b \in \mathcal{A}(\tau), f \in \ell^{2}(\tau)$, where $\chi$ stands for the characteristic function of the set $S\left(I^{\text {fin }}\right)$. In particular, $\lambda_{0}\left(\delta_{i}\right)=\rho_{0}\left(\delta_{i}\right)=0$ whenever $i \in I \backslash I^{\text {fin }}$.

We will study two corresponding *-subalgebras of $\mathcal{B}\left(\ell^{2}(\tau)\right)$, namely

$$
\mathcal{L}:=\lambda_{0}(\mathcal{A}(\tau)) \quad \text { and } \quad \mathcal{R}:=\rho_{0}(\mathcal{A}(\tau)),
$$

and the von Neumann algebras which are their commutants:

$$
\begin{aligned}
& \mathcal{S}:=\mathcal{R}^{\prime}=\left\{A \in \mathcal{B}\left(\ell^{2}(\tau)\right): A B=B A \text { for every } B \in \mathcal{R}\right\}, \\
& \mathcal{T}:=\mathcal{L}^{\prime}=\left\{B \in \mathcal{B}\left(\ell^{2}(\tau)\right): A B=B A \text { for every } A \in \mathcal{L}\right\} .
\end{aligned}
$$

The aim of this section is to show that every minimal $\mathcal{L}$-invariant closed subspace of $\ell^{2}(\tau)$ is one-dimensional.

Lemma 2.1. If $f, g \in \ell^{2}(\tau)$ then the function $f *_{\tau} g$ is well defined. Moreover, for every $u \in S(I)$ there is a constant $C(u)$ such that

$$
\left|\left(f *_{\tau} g\right)(u)\right| \leq C(u)\|f\|_{2} \cdot\|g\|_{2}
$$

for every $f, g \in \ell^{2}(\tau)$. 
Proof. For $u \in S(I) \backslash S\left(I^{\text {fin }}\right)$ we can put $C(u)=0$. Fix $u=i_{1} \ldots i_{m} \in S\left(I^{\text {fin }}\right)$. Then

$$
\begin{aligned}
& \left(f *_{\tau} g\right)(u)=\sum_{k=0}^{m} \sum_{\substack{j_{1} \ldots j_{n} \in S\left(I^{\mathrm{fin}}\right) \\
j_{n} \neq i_{k}, i_{k+1}}} f\left(i_{1} \ldots i_{k} j_{n} \ldots j_{1}\right) g\left(j_{1} \ldots j_{n} i_{k+1} \ldots i_{m}\right) \tau\left(j_{1} \ldots j_{n}\right) \\
& +\sum_{k=1}^{m} \sum_{\substack{j_{1} \ldots j_{n} \in S\left(I^{\mathrm{fin}}\right) \\
j_{n} \neq i_{k}}} f\left(i_{1} \ldots i_{k} j_{n} \ldots j_{1}\right) g\left(j_{1} \ldots j_{n} i_{k} i_{k+1} \ldots i_{m}\right) \tau\left(j_{1} \ldots j_{n}\right)\left(1-\tau_{i_{k}}\right) .
\end{aligned}
$$

Therefore, putting $c_{1}(u)=\max \left\{\left|1-\tau_{i_{k}}\right| \tau_{i_{k}}^{-1 / 2}: k=1, \ldots, m\right\}$, we get

$$
\begin{aligned}
&\left|\left(f *_{\tau} g\right)(u)\right| \leq \frac{1}{\sqrt{\tau(u)}} \sum_{\substack { k=0 \\
\begin{subarray}{c}{j_{1} \ldots j_{n} \in S\left(I^{\mathrm{fin}}\right) \\
j_{n} \neq i_{k}, i_{k+1}{ k = 0 \\
\begin{subarray} { c } { j _ { 1 } \ldots j _ { n } \in S ( I ^ { \mathrm { fin } } ) \\
j _ { n } \neq i _ { k } , i _ { k + 1 } } }\end{subarray}}^{m} \mid f\left(i_{1} \ldots i_{k} j_{n} \ldots j_{1}\right) \sqrt{\tau\left(i_{1} \ldots i_{k} j_{n} \ldots j_{1}\right)} \\
& \times g\left(j_{1} \ldots j_{n} i_{k+1} \ldots i_{m}\right) \sqrt{\tau\left(j_{1} \ldots j_{n} i_{k+1} \ldots i_{m}\right)} \mid \\
&+\frac{1}{\sqrt{\tau(u)}} \sum_{k=1}^{m} \sum_{\substack{j_{1} \ldots j_{n} \in S\left(I^{\mathrm{fin}}\right) \\
j_{n} \neq i_{k}}} \frac{\left|1-\tau_{i_{k}}\right|}{\sqrt{\tau_{i_{k}}}} \mid f\left(i_{1} \ldots i_{k} j_{n} \ldots j_{1}\right) \sqrt{\tau\left(i_{1} \ldots i_{k} j_{n} \ldots j_{1}\right)} \\
& \quad \times g\left(j_{1} \ldots j_{n} i_{k} i_{k+1} \ldots i_{m}\right) \sqrt{\tau\left(j_{1} \ldots j_{n} i_{k} i_{k+1} \ldots i_{m}\right)} \mid \\
& \leq \frac{1}{\sqrt{\tau(u)}}\|f\|_{2} \cdot\|g\|_{2}+\frac{c_{1}(u)}{\sqrt{\tau(u)}\|f\|_{2} \cdot\|g\|_{2},}
\end{aligned}
$$

which concludes the proof.

Knowing that the map $f \mapsto \widetilde{f}=\sum_{u \in S(I)} f(u) \mu_{u}$ (formula $\left.(4.2)\right)$ of $\mathcal{A}(\tau)$ onto $\mathcal{F}_{t}(G)$ preserves the $\ell^{2}$-norm: $\|\widetilde{f}\|_{\ell^{2}(\tau)}=\|f\|_{\ell^{2}(G)}$, we note that if $\tau_{i} \in\{1,1 / 2,1 / 3, \ldots\}$ for every $i \in I^{\text {fin }}$ then one can take $C(u)=\tau^{-1}(u)$ for $u \in S\left(I^{\text {fin }}\right)$.

We will use the following facts:

\section{Lemma 2.2.}

1. If $f, f_{n}, g, g_{n} \in \ell^{2}(\tau)$ and if $\left\|f_{n}-f\right\|_{2} \rightarrow 0$, $\left\|g_{n}-g\right\|_{2} \rightarrow 0$ then $\left(f_{n} *_{\tau} g_{n}\right)(u) \rightarrow$ $\left(f *_{\tau} g\right)(u)$ for every $u \in S(I)$.

2. If $f, g, h \in \ell^{2}(\tau)$ and if one of them has finite support then

$$
\left(f *_{\tau} g\right) *_{\tau} h=f *_{\tau}\left(g *_{\tau} h\right)
$$

in particular

$$
\left[f *_{\tau} g, h^{*}\right]=\left[f, h^{*} *_{\tau} g^{*}\right]=\left[g, f^{*} *_{\tau} h^{*}\right] .
$$

Proof. For (1) we observe that

$$
\begin{gathered}
\left|f_{n} *_{\tau} g_{n}(u)-f *_{\tau} g(u)\right| \leq\left|\left(f_{n} *_{\tau}\left(g_{n}-g\right)\right)(u)\right|+\left|\left(\left(f_{n}-f\right) *_{\tau} g\right)(u)\right| \\
\leq C(u)\left\|f_{n}\right\|_{2} \cdot\left\|g_{n}-g\right\|_{2}+C(u)\left\|f_{n}-f\right\|_{2} \cdot\|g\|_{2} .
\end{gathered}
$$

To prove (2) assume that $h \in \mathcal{F}^{\text {fin }}(S(I))$ and take sequences $f_{n}, g_{n} \in \mathcal{F}^{\text {fin }}(S(I)) \cap \ell^{2}(\tau)$ such that $\left\|f_{n}-f\right\|_{2} \rightarrow 0,\left\|g_{n}-g\right\|_{2} \rightarrow 0$. Then for fixed $u \in S(I)$ we have

$$
\left(\left(f_{n} *_{\tau} g_{n}\right) *_{\tau} h\right)(u)=\left(f_{n} *_{\tau}\left(g_{n} *_{\tau} h\right)\right)(u) .
$$


Since $\left(f_{n} *_{\tau} g_{n}\right)(v) \rightarrow\left(f *_{\tau} g\right)(v)$ for every $v \in S(I)$ and $h$ has finite support we have

$$
\left(\left(f_{n} *_{\tau} g_{n}\right) *_{\tau} h\right)(u) \rightarrow\left(\left(f *_{\tau} g\right) *_{\tau} h\right)(u) .
$$

We also have $\left\|g_{n} *_{\tau} h-g *_{\tau} h\right\|_{2} \rightarrow 0$ so that part (1) implies that

$$
\left(f_{n} *_{\tau}\left(g_{n} *_{\tau} h\right)\right)(u) \rightarrow\left(f *_{\tau}\left(g *_{\tau} h\right)\right)(u) .
$$

The other cases can be proved similarly.

Lemma 2.3. For $a \in \ell^{2}(\tau)$ define operators $\lambda_{0}(a)$ and $\rho_{0}(a)$, with domains $\mathcal{D}\left(\lambda_{0}(a)\right)$ and $\mathcal{D}\left(\rho_{0}(a)\right)$, as the closure of the maps

$$
\mathcal{F}^{\mathrm{fin}}(S(I)) \ni f \mapsto a *_{\tau} f \quad \text { and } \quad \mathcal{F}^{\mathrm{fin}}(S(I)) \ni f \mapsto f *_{\tau} a^{*}
$$

respectively. Then $\lambda_{0}\left(a^{*}\right) \subseteq \lambda_{0}(a)^{*}$ and $\rho_{0}\left(a^{*}\right) \subseteq \rho_{0}(a)^{*}$. Moreover, if $g \in \mathcal{D}\left(\lambda_{0}(a)^{*}\right)$ $\left(\right.$ resp. $\left.g_{1} \in \mathcal{D}\left(\rho_{0}(a)^{*}\right)\right)$ then $\lambda_{0}(a)^{*} g=a^{*} *_{\tau} g$ (resp. $\left.\rho_{0}(a)^{*} g_{1}=g_{1} *_{\tau} a\right)$.

Proof. First we note that in view of Lemma 2.1 the maps $\mathcal{F}^{\text {fin }}(S(I)) \ni f \mapsto a *_{\tau} f$ and $\mathcal{F}^{\text {fin }}(S(I)) \ni f \mapsto f *_{\tau} a^{*}$ are closable.

Assume that $(g, h) \in \lambda_{0}\left(a^{*}\right)$. Then there is a sequence $g_{n} \in \mathcal{F}^{\mathrm{fin}}(S(I))$ such that $g_{n} \rightarrow g$ and $a^{*} *_{\tau} g_{n} \rightarrow h$ in $\ell^{2}(\tau)$. Then, by Lemma 2.2, for $f \in \mathcal{F}^{\text {fin }}(S(I))$ we have

$$
\left[\lambda_{0}(a) f, g\right]=\lim _{n \rightarrow \infty}\left[a *_{\tau} f, g_{n}\right]=\lim _{n \rightarrow \infty}\left[f, a^{*} *_{\tau} g_{n}\right]=[f, h],
$$

which means that $(g, h) \in \lambda_{0}(a)^{*}$.

Now, if $g \in \mathcal{D}\left(\lambda_{0}(a)^{*}\right)$ and $\lambda_{0}(a)^{*} g=h$ then, by definition of adjoint and by Lemma 2.2,

$$
\left[\delta_{u}, h\right]=\left[\lambda_{0}(a) \delta_{u}, g\right]=\left[a *_{\tau} \delta_{u}, g\right]=\left[\delta_{u}, a^{*} *_{\tau} g\right],
$$

which means that $h=a^{*} *_{\tau} g$.

\section{Proposition 2.4.}

1. If $A \in \mathcal{S}$ then there exists a function $a \in \ell^{2}(\tau)$ such that $A(f)=a *_{\tau} f$ for $f \in \ell^{2}(\tau)$. Similarly, if $B \in \mathcal{T}$ then there exists a function $b \in \ell^{2}(\tau)$ such that $B(f)=f *_{\tau} b^{*}$ for $f \in \ell^{2}(\tau)$.

2. $\mathcal{S}$ is the weak closure of $\mathcal{L}$ and $\mathcal{T}$ is the weak closure of $\mathcal{R}$.

3. The map $A \mapsto \operatorname{Tr}(A):=\left[A \delta_{e}, \delta_{e}\right]$ is a faithful tracial state on $\mathcal{S}$ (and on $\mathcal{T}$ ).

Proof. 1) Fix $A \in \mathcal{S}$ and put $a:=A\left(\delta_{e}\right)$. If $f \in \mathcal{F}^{\mathrm{fin}}(S(I))$ then by the definition of $\mathcal{S}$

$$
A(f)=A\left(\delta_{e} *_{\tau} f\right)=A\left(\rho_{0}\left(f^{*}\right)\left(\delta_{e}\right)\right)=\rho_{0}\left(f^{*}\right)\left(A\left(\delta_{e}\right)\right)=\left(\rho_{0}\left(f^{*}\right)\right)(a)=a *_{\tau} f .
$$

In order to prove this equality for all $f \in \ell^{2}(\tau)$ we define functionals $\phi_{u}(f):=(A f)(u)$, $u \in S\left(I^{\text {fin }}\right)$. Since $\tau(u)|f(u)|^{2} \leq\|f\|_{2}^{2}$ we have $\left\|\phi_{u}\right\| \leq\|A\| / \sqrt{\tau(u)}$. Hence if $f_{n} \in$ $\mathcal{F}^{\text {fin }}(S(I))$ and $\left\|f_{n}-f\right\|_{2} \rightarrow 0$ then

$$
(A f)(u)=\lim \left(A f_{n}\right)(u)=\lim \left(a *_{\tau} f_{n}\right)(u),
$$

so $a *_{\tau} f$ is a well defined function equal to $A f$.

2) Note that $\mathcal{S}$ is weakly closed and $\mathcal{L} \subseteq \mathcal{S}$, hence the weak closure of $\mathcal{L}$ is contained in $\mathcal{S}$. On the other hand, if $A \in \mathcal{S}, B \in \mathcal{T}$ then $A B=B A$ in view of the previous point and Lemma 2.2. Therefore $\mathcal{S} \subseteq \mathcal{T}^{\prime}=\mathcal{L}^{\prime \prime}$, which is equal to the weak closure of $\mathcal{L}$ by the von Neumann theorem.

3) Take $A, B \in \mathcal{S}$ and put $a=A\left(\delta_{e}\right), b=B\left(\delta_{e}\right) \in \ell^{2}(\tau)$. Then

$\operatorname{Tr}(A B)=\left[A B \delta_{e}, \delta_{e}\right]=\left[a *_{\tau}\left(b *_{\tau} \delta_{e}\right), \delta_{e}\right]=\left(a *_{\tau} b\right)(e)=\left(b *_{\tau} a\right)(e)=\left[B A \delta_{e}, \delta_{e}\right]=\operatorname{Tr}(B A)$. 
If $0 \leq A \in \mathcal{S}$ then $A=C^{*} C$ for some $C \in \mathcal{S}$, hence putting $c=C\left(\delta_{e}\right)$ we have

$$
\operatorname{Tr}(A)=\left[C^{*} C \delta_{e}, \delta_{e}\right]=\left(c^{*} *_{\tau} c\right)(e)=\|c\|_{2}^{2},
$$

which concludes the proof.

From now on we fix a minimal nontrivial closed $\mathcal{L}$-invariant subspace $V \subseteq \ell^{2}(\tau)$ and a minimal biinvariant (i.e. both $\mathcal{L}$ - and $\mathcal{R}$-invariant) closed subspace $W$ containing $V$.

Lemma 2.5. The subspace $W$ can be decomposed into the orthogonal sum $W=\bigoplus_{\alpha} V_{\alpha}$, with $V$ as one of the summands and where each $V_{\alpha}$ is closed, $\mathcal{L}$-invariant and the restriction of $\lambda_{0}$ to $V_{\alpha}$ is equivalent to the restriction of $\lambda_{0}$ to $V$.

Proof. Let $W_{0}$ be a maximal orthogonal sum of the form $\bigoplus_{\alpha} V_{\alpha}$, such that $V$ is one of the summands, $V_{\alpha} \subseteq W$ is $\lambda_{0}$-invariant and the restriction of $\lambda_{0}$ to $V_{\alpha}$ is equivalent to the restriction of $\lambda_{0}$ to $V$. Denote by $P$ the orthogonal projection of $\ell^{2}(\tau)$ onto $W_{0}^{\perp}$. Since this subspace is $\mathcal{L}$-invariant we have $A P=P A$ for every $A \in \mathcal{L}$.

Assume that $W_{0}$ is not $\mathcal{R}$ invariant, i.e. there is $b \in \mathcal{A}(\tau)$ and an index $\beta$ such that $V_{\beta} *_{\tau} b \nsubseteq W_{0}$. Define an operator $B: V_{\beta} \rightarrow W_{0}^{\perp}$ by $B(f):=P\left(f *_{\tau} b\right)$. Note that $f *_{\tau} b$, and hence $B(f)$, belongs to $W$ and that $A B(f)=B A(f)$ for $f \in V_{\beta}, A \in \mathcal{L}$. Indeed, if $A=\lambda_{0}(a)$ then

$$
A B(f)=A P\left(f *_{\tau} b\right)=P A\left(f *_{\tau} b\right)=P\left(a *_{\tau} f *_{\tau} b\right)=B A(f) .
$$

We claim that $B$ is a multiple of an isometry $V_{\beta} \rightarrow V^{\prime}:=B\left(V_{\beta}\right)$. To see this take the polar decomposition $B=U D$. By definition $D=\sqrt{B^{*} B}$. For $a \in \mathcal{A}(\tau)$ and $\xi \in V_{\beta}$

$$
\lambda_{0}(a) B^{*} B \xi=B^{*} \lambda_{0}(a) B \xi=B^{*} B \lambda_{0}(a) \xi,
$$

so by the Schur lemma $B^{*} B$, and hence $D$, is a nonzero scalar multiple of the identity. Now $U$, defined by $U D \xi:=B \xi$ for $\xi \in V_{\beta}$, is obviously a unitary operator $V_{\beta} \rightarrow V^{\prime}$, satisfying $U \lambda_{0}(a) \xi=\lambda_{0}(a) U \xi$ for $a \in \mathcal{A}(\tau), \xi \in V_{\beta}$. Therefore $V^{\prime}$ can be added to the sum $\oplus_{\alpha} V_{\alpha}$, which is a contradiction.

Lemma 2.6. The subspace $V$ has finite dimension.

Proof. Let $\mathcal{L}_{W}=\left\{\left.A\right|_{W}: A \in \mathcal{L}\right\}, \mathcal{S}_{W}=\left\{\left.A\right|_{W}: A \in \mathcal{S}\right\}, \mathcal{L}_{V}=\left\{\left.A\right|_{V}: A \in \mathcal{L}\right\}$ and $\mathcal{S}_{V}=\left\{\left.A\right|_{V}: A \in \mathcal{S}\right\}$. By the previous lemma $\mathcal{L}_{W} \cong \mathcal{L}_{V}, \mathcal{S}_{W} \cong \mathcal{S}_{V}$ as $*$-algebras (cf. A20 in the Appendix of [Di]) and by the Schur lemma combined with the von Neumann theorem we have $\mathcal{S}_{V}=\mathcal{B}(V)$.

For $X \in \mathcal{S}_{W}$ we define an operator $\tilde{X}$ on $\ell^{2}(\tau)$ by $\tilde{X}:=X Q$, where $Q$ denotes the orthogonal projection of $\ell^{2}(\tau)$ onto $W$. Note that $B \tilde{X}=\tilde{X} B$ for any $B \in \mathcal{R}$. Indeed, if $X=\left.\lambda_{0}(x)\right|_{W}, B=\rho_{0}(b)$, with $x, b \in \mathcal{A}(\tau)$, then for $f=f_{1}+f_{2} \in \ell^{2}(\tau)$, with $f_{1}=Q f$, we have

and

$$
B \tilde{X} f=B X f_{1}=B\left(x *_{\tau} f_{1}\right)=x *_{\tau} f_{1} *_{\tau} b^{*}
$$

$$
\tilde{X} B f=X Q B f=X Q B f=X B f_{1}=X\left(f_{1} *_{\tau} b^{*}\right)=x *_{\tau} f_{1} *_{\tau} b^{*}
$$

because $W$ is bi-invariant. Moreover $X \mapsto \tilde{X}$ is a $*$-homomorphism of $\mathcal{S}_{W}$ into $\mathcal{S}$. Therefore we can define a tracial state on $\mathcal{S}_{W} \cong \mathcal{B}(V)$ by $\tilde{\operatorname{Tr}}(X):=\operatorname{Tr}(\tilde{X})$ (Tr was defined in Proposition 2.4). But if $\operatorname{dim} V$ is infinite then there is no tracial state on $\mathcal{B}(V)$ because we can take an orthogonal decomposition $V=V_{1} \oplus V_{2}$ and partial isometries $C_{1}, C_{2}$ of $V$ onto $V_{1}, V_{2}$ respectively such that $C_{1} C_{1}^{*}+C_{2} C_{2}^{*}=\mathrm{Id}$ and $C_{1}^{*} C_{1}+C_{2}^{*} C_{2}=2 \mathrm{Id}$, which excludes existence of a tracial state on $\mathcal{B}(V)$ for infinite dimensional $V$. 
Lemma 2.7. For a function $a \in \ell^{2}(\tau)$ the following conditions are equivalent:

1. $\lambda_{0}(a)$ is a bounded operator,

2. $\lambda_{0}\left(a^{*}\right)$ is a bounded operator,

3. $\rho_{0}(a)$ is a bounded operator,

4. $\rho_{0}\left(a^{*}\right)$ is a bounded operator,

5. $a=A\left(\delta_{e}\right)$ for some $A \in \mathcal{S}$,

6. $a^{*}=B\left(\delta_{e}\right)$ for some $B \in \mathcal{T}$.

Proof. In view of Lemma 2.3 we have $(1) \Leftrightarrow(2)$ and $(3) \Leftrightarrow(4)$ and by the first part of Proposition 2.4 we have (1) $\Leftrightarrow$ (5) and (3) $\Leftrightarrow(6)$. To conclude, we note that $\left\|a *_{\tau} f\right\|_{2}=\left\|f^{*} *_{\tau} a^{*}\right\|_{2}$ for $f \in \ell^{2}(\tau)$, which means that $(1) \Leftrightarrow(3)$.

Definition 2.8. A function $a \in \ell^{2}(\tau)$ is said to be moderated if satisfies conditions of the previous lemma. Note that moderated functions constitute a $*$-algebra and if $a, b$ are moderated then $\lambda_{0}\left(a *_{\tau} b\right)=\lambda_{0}(a) \lambda_{0}(b)$ and $\lambda_{0}(a)^{*}=\lambda_{0}\left(a^{*}\right)$.

Lemma 2.9. Every function $f \in V$ is moderated.

Proof. Let $P$ denotes the orthogonal projection of $\ell^{2}(\tau)$ onto $V$. Since $P \in \mathcal{L}^{\prime}=\mathcal{T}$ we have $P(g)=g *_{\tau} k$ for some moderated function $k$ such that $k=k *_{\tau} k=k^{*}$. Hence if $g$ is a moderated function then so is $P(g)=g *_{\tau} k$. Moderated functions form a dense linear subspace $\mathcal{M}$ of $\ell^{2}(\tau)$, hence $\mathcal{M} \cap V=P(\mathcal{M})$ is a dense subspace of $V$. But $V$ has finite dimension, which implies that $\mathcal{M} \cap V=V$.

Lemma 2.10. Suppose that $f, g \in V$ and set $\phi(u)=\left[\lambda_{0}\left(\delta_{u}\right) f, g\right]$. Then there is $f_{0} \in$ $\ell^{2}(\tau)$ such that $\phi(u)=f_{0}(u) \tau(u)$ for every $u \in S(I)$.

Proof. By Lemma 2.2 we have

$$
\phi(u)=\left[\lambda_{0}\left(\delta_{u}\right) f, g\right]=\left(\delta_{u} *_{\tau}\left(f *_{\tau} g^{*}\right)\right)(e)=\left(f *_{\tau} g^{*}\right)\left(u^{*}\right) \cdot \tau(u) .
$$

By the previous lemma $f$ and $g$ are moderated, hence so is $f *_{\tau} g^{*}$, therefore $f *_{\tau} g^{*} \in$ $\ell^{2}(\tau)$.

Now we are able to prove the main result of this section.

Theorem 2.11. If $V$ is an $\mathcal{L}$-invariant minimal nontrivial subspace of $\ell^{2}(\tau)$ then $\operatorname{dim} V=1$.

Proof. We know already from Lemma 2.6 that $\operatorname{dim} V<\infty$. It is sufficient to prove that all the operators $\left.\lambda_{0}\left(\delta_{i}\right)\right|_{V}, i \in I$, commute.

Assume that $\left|I^{\text {fin }}\right| \geq 2$ and fix $I_{0} \subseteq I^{\text {fin }}$ with $\left|I_{0}\right|=2$, say $I_{0}=\{1,2\}$. Denote by $\mathcal{A}_{0}$ the unital $*$-subalgebra of $\mathcal{A}(\tau)$ generated by $\left\{\delta_{1}, \delta_{2}\right\}$. Set also $\mathcal{L}_{0}:=\left\{\lambda_{0}(a): a \in \mathcal{A}_{0}\right\}$. Now decompose $V$ into an orthogonal direct sum of minimal $\mathcal{L}_{0}$-invariant subspaces:

$$
V=V_{1} \oplus V_{2} \oplus \cdots \oplus V_{s}
$$

The *-algebra $\mathcal{A}_{0}$ is isomorphic to the convolution $*$-algebra of finitely supported functions on the dihedral group $\mathbf{Z}_{2} * \mathbf{Z}_{2}$. This group can be represented as the semidirect product $\mathbb{Z}_{2} \ltimes \mathbb{Z}$, which implies that $\operatorname{dim} V_{r} \leq 2$ for $r \leq s$ (see Lemma 7.1). We only need to show that all $V_{r}$ have dimension one.

Suppose that $\operatorname{dim} V_{r}=2$ for some $r \leq s$ and denote $A_{i}:=\left.\lambda_{0}\left(\delta_{i}\right)\right|_{V_{r}}, i=1,2$. Then we have $A_{i}=\left(1+\tau_{i}\right) P_{i}-\tau_{i} \mathrm{Id}$, where $P_{i}$ is an orthogonal projection on $V_{r}$. Due to minimality of $V_{r}$ both projections have one-dimensional images. Therefore $\operatorname{det} A_{i}=-\tau_{i}$ and $\operatorname{det}\left(A_{1} A_{2}\right)=\tau_{1} \tau_{2}$. Hence the operator $A_{1} A_{2}$ has an eigenvalue $\gamma_{0}$ satisfying $\left|\gamma_{0}\right| \geq$ 
$\sqrt{\tau_{1} \tau_{2}}$. Now take the corresponding unit eigenvector $\xi_{0} \in V_{r}$ and consider the function $\phi$ on $S(I)$ given by: $\phi(u)=\left[\lambda_{0}\left(\delta_{u}\right) \xi_{0}, \xi_{0}\right]$. Then for $u=1212 \ldots 12$, with $|u|=2 m$, we have

$$
\phi(u)=\left|\left[\left(A_{1} A_{2}\right)^{m} \xi_{0}, \xi_{0}\right]\right|=\left|\gamma_{0}^{m}\right| \geq\left(\sqrt{\tau_{1} \tau_{2}}\right)^{m},
$$

which implies that the function $S\left(I_{0}\right) \ni u \mapsto \phi(u) / \tau(u)$ does not belong to $\ell^{2}\left(S\left(I_{0}\right), \tau\right)$. This contradicts the last lemma and therefore proves that $\operatorname{dim} V_{r}=1$ for every $r \leq s$. Therefore the operators $\left.\lambda_{0}\left(\delta_{1}\right)\right|_{V}$ and $\left.\lambda_{0}\left(\delta_{2}\right)\right|_{V}$ do commute.

Let us now describe one dimensional $\lambda_{0}$-invariant subspaces of $\ell^{2}(\tau)$ and the corresponding $\tau$-positive definite functions.

For $i \in I$ we have the partition $S(I)=S_{i} \dot{\cup} i S_{i}$, where $S_{i}$ was defined in (1.2), and the orthogonal decomposition $\ell^{2}(\tau)=M_{i} \oplus N_{i}$, where

$$
\begin{aligned}
& M_{i}=\left\{f_{1} \in \ell^{2}(\tau): f_{1}(u)=\tau_{i} f_{1}(i u) \text { for every } u \in S_{i}\right\}, \\
& N_{i}=\left\{f_{2} \in \ell^{2}(\tau): f_{2}(u)+f_{2}(i u)=0 \text { for every } u \in S_{i}\right\} .
\end{aligned}
$$

Indeed, for $f_{1} \in M_{i}, f_{2} \in N_{i}$ we have

$$
\left[f_{1}, f_{2}\right]=\sum_{u \in S(I)} f_{1}(u) \overline{f_{2}(u)} \tau(u)=\sum_{u \in S_{i}}\left(f_{1}(u) \overline{f_{2}(u)} \tau(u)+f_{1}(i u) \overline{f_{2}(i u)} \tau(i u)\right)=0 .
$$

On the other hand, every $f \in \ell^{2}(\tau)$ can be decomposed as $f=f_{1}+f_{2}, f_{1} \in M_{i}, f_{2} \in N_{i}$ where for $u \in S_{i}$

$$
\begin{aligned}
f_{1}(u) & =\frac{\tau_{i}}{1+\tau_{i}}(f(u)+f(i u)), \\
f_{1}(i u) & =\frac{1}{1+\tau_{i}}(f(u)+f(i u)), \\
f_{2}(u) & =\frac{1}{1+\tau_{i}}\left(f(u)-\tau_{i} f(i u)\right), \\
f_{2}(i u) & =\frac{1}{1+\tau_{i}}\left(\tau_{i} f(i u)-f(u)\right) .
\end{aligned}
$$

Consider the operator $A_{i}:=\lambda_{0}\left(\delta_{i}\right)$ on $\ell^{2}(\tau)$. For $f=\sum_{u \in S(I)} f(u) \delta_{u}$ we have

$$
\delta_{i} *_{\tau} f=\sum_{u \in S_{i}}\left(f(u) \delta_{i u}+\left(1-\tau_{i}\right) f(i u) \delta_{i u}+\tau_{i} f(i u) \delta_{u}\right) .
$$

Therefore, if $f \in M_{i}$ then $A_{i} f=f$ while for $f \in N_{i}$ we have $A_{i} f=-\tau_{i} f$.

If a function $f: S(I) \rightarrow \mathbb{C}$, supported on $S\left(I^{\text {fin }}\right)$, is an eigenfunction for every $A_{i}$, $i \in I$, then, up to a constant, $f\left(i_{1} i_{2} \ldots i_{n}\right)=f\left(i_{1}\right) f\left(i_{2}\right) \ldots f\left(i_{n}\right)$, where $f(i) \in\left\{\tau_{i}^{-1},-1\right\}$ for $i \in I^{\text {fin }}$. In view of Lemma 1.5 in [FS] such a function belongs to $\ell^{2}(\tau)$ if and only if

$$
\sum_{i \in I_{1}} \frac{1}{1+\tau_{i}}+\sum_{i \in I_{2}} \frac{\tau_{i}}{1+\tau_{i}}<1
$$

where $I_{1}:=\left\{i \in I^{\mathrm{fin}} \mid f(i)=\tau_{i}^{-1}\right\}, I_{2}:=\left\{i \in I^{\mathrm{fin}} \mid f(i)=-1\right\}$. If this holds then the corresponding $\tau$-positive definite function

$$
\phi\left(i_{1} i_{2} \ldots i_{m}\right)=\left[A_{i_{1}} A_{i_{2}} \ldots A_{i_{m}} f, f\right]
$$

is, up to a constant, the character of $\mathcal{A}(\tau)$ given by

$$
\phi\left(i_{1} i_{2} \ldots i_{m}\right)=\phi\left(i_{1}\right) \phi\left(i_{2}\right) \ldots \phi\left(i_{m}\right),
$$


where

$$
\phi(i)= \begin{cases}1 & \text { if } f(i)=\tau_{i}^{-1}, \\ -\tau_{i} & \text { if } f(i)=-1 .\end{cases}
$$

For example, the trivial representation of $\mathcal{A}(\tau)$, corresponding to the character $\phi \equiv 1$, is contained in the regular representation if and only if $\sum_{i \in I} 1 /\left(1+\tau_{i}\right)<1$.

Now we make the following elementary observation:

Lemma 2.12. Suppose that for every $i \in I$ we have $0 \leq \alpha_{i}^{0} \leq \alpha_{i}^{1} \leq 1, \alpha_{i}^{0}+\alpha_{i}^{1}=1$ and that $\sum_{i \in I} \alpha_{i}^{\epsilon_{i}}<1$ for some $\epsilon: I \rightarrow\{0,1\}$. Then either $\epsilon_{i}=0$ for all $i \in I$ or there is $j \in I$ such that $\alpha_{j}^{0}>\alpha_{i}^{0}$ for every $i \in I \backslash\{j\}, \epsilon_{j}=1$ and $\epsilon_{i}=0$ for all $i \neq j$.

Putting $\alpha_{i}^{0}:=\min \left\{1 /\left(1+\tau_{i}\right), \tau_{i} /\left(1+\tau_{i}\right)\right\}$ we obtain

Corollary 2.13. The space $\ell^{2}(\tau)$ contains at most two one-dimensional $\mathcal{L}$-invariant subspaces.

The next result will be needed later on.

Theorem 2.14. Assume that $a \in \ell^{2}(\tau)$ is such that the function $u \mapsto a(u) \tau(u)$ is $\tau$ positive definite. Then there is $c=c^{*} \in \ell^{2}(\tau)$ such that $a=c *_{\tau} c$ and $c \cdot \tau$ is $\tau$-positive definite. Moreover, the GNS representation related to $a \cdot \tau$ is contained in $\lambda_{0}$.

The proof, similarly as for groups (see [Di]), is based on the following lemmas.

Lemma 2.15. Assume that a is a moderated function. Then the operator $\lambda_{0}(a)$ is nonnegative if and only if the function $u \mapsto a(u) \tau(u)$ is $\tau$-positive definite.

Proof. For $f \in \mathcal{F}^{\text {fin }}(S(I))$ we have

$$
\left[\lambda_{0}(a) f, f\right]=\left(a *_{\tau}\left(f *_{\tau} f^{*}\right)\right)(e)=\sum_{u \in S(I)} a(u)\left(f *_{\tau} f^{*}\right)(u) \tau(u)=\left\langle a \cdot \tau, f *_{\tau} f^{*}\right\rangle .
$$

Lemma 2.16. Assume that $a, b$ are moderated functions such that $a *_{\tau} b=b *_{\tau} a$ and $0 \leq \lambda_{0}(a) \leq \lambda_{0}(b)$. Then

$$
\|b-a\|_{2}^{2} \leq\|b\|_{2}^{2}-\|a\|_{2}^{2}
$$

Proof. Put $c=b-a$. Since the operator $\lambda_{0}\left(a *_{\tau} c\right)=\lambda_{0}(a) \lambda_{0}(c)$ is nonnegative we have $[a, c]=\left(a *_{\tau} c\right)(e) \geq 0$, so $[a, a] \leq[a, a]+[a, c]=[a, b]$ which gives

$$
\|b-a\|_{2}^{2}=\|b\|_{2}^{2}+\|a\|_{2}^{2}-2[a, b] \leq\|b\|_{2}^{2}-\|a\|_{2}^{2} .
$$

Lemma 2.17. Assume that $a_{1}, a_{2}, \ldots$ are pairwise commuting moderated functions such that

$$
0 \leq \lambda_{0}\left(a_{1}\right) \leq \lambda_{0}\left(a_{2}\right) \leq \ldots
$$

and $\sup \left\|a_{n}\right\|_{2}<\infty$. Then there is $a \in \ell^{2}(\tau)$ such that $\left\|a-a_{n}\right\|_{2} \rightarrow 0$.

Proof. In view of the previous lemma the sequence $\left\|a_{n}\right\|_{2}$ is increasing and $a_{n}$ is a Cauchy sequence in $\ell^{2}(\tau)$.

Proof of Theorem 2.14. If $a$ is moderated then the proof goes as in Theorem 13.8.6 in [Di]. For the general case let $T$ denote the Friedrichs extension of the nonnegative operator $\rho_{0}(a)=\rho_{0}\left(a^{*}\right)$. Since the operators

$$
U_{i}:=\lambda_{0}\left(\frac{2}{1+\tau_{i}} \delta_{i}-\frac{1-\tau_{i}}{1+\tau_{i}} \delta_{e}\right)
$$


are unitary we note that (cf. Lemma 13.8.3 in [Di]):

i. $U_{i} T=T U_{i}$ for $i \in I$ and therefore $\lambda_{0}\left(\delta_{u}\right) T=T \lambda_{0}\left(\delta_{u}\right)$ for $u \in S(I)$,

ii. $T h=h *_{\tau}$ a for every $h$ in the domain of $T$.

(The second statement holds by Lemma 2.3 as $T \subseteq \lambda_{0}(a)^{*}$.) Take the spectral resolution $T=\int_{0}^{\infty} \zeta d E_{\zeta}$ of $T$. Then the projections $E_{\zeta}$ commute with all $\lambda_{0}\left(\delta_{u}\right), u \in S(I)$. Put $a_{\zeta}:=E_{\zeta} a \in \ell^{2}(\tau)$. For $g \in \mathcal{A}(\tau), u \in S(I)$ we have

$$
\begin{gathered}
{\left[g *_{\tau} a_{\zeta}^{*}, \delta_{u}\right]=\left[g, \delta_{u} *_{\tau} a_{\zeta}\right]=\left[g, \delta_{u} *_{\tau} E_{\zeta} a\right]} \\
=\left[g, E_{\zeta}\left(\delta_{u} *_{\tau} a\right)\right]=\left[E_{\zeta} g, \delta_{u} *_{\tau} a\right]=\left[E_{\zeta} g *_{\tau} a^{*}, \delta_{u}\right] .
\end{gathered}
$$

This means that

$$
g *_{\tau} a_{\zeta}^{*}=\left(E_{\zeta} g\right) *_{\tau} a^{*}=\left(E_{\zeta} g\right) *_{\tau} a=T E_{\zeta} g
$$

which implies that $a_{\zeta}^{*}$ is moderated, $a_{\zeta}^{*}=a_{\zeta}$ and $a \cdot \tau$ is $\tau$-positive definite. The rest of the proof goes as for 13.8.6 in [Di].

For the second part we write

$$
\left[\lambda_{0}\left(\delta_{u}\right) c, c\right]=\left(\left(\delta_{u} *_{\tau} c\right) *_{\tau} c\right)(e)=\left(\delta_{u} *_{\tau}\left(c *_{\tau} c\right)\right)(e)=\left(\delta_{u} *_{\tau} a\right)(e)=a(u) \cdot \tau(u) .
$$

We conclude this section with two propositions which will not be used in the sequel.

Proposition 2.18. Assume that $a, b, c \in \ell^{2}(\tau)$.

1. If $a$ is moderated and $b *_{\tau} c \in \ell^{2}(\tau)$ then $a *_{\tau}\left(b *_{\tau} c\right)=\left(a *_{\tau} b\right) *_{\tau} c$.

2. If $b$ is moderated then $a *_{\tau}\left(b *_{\tau} c\right)=\left(a *_{\tau} b\right) *_{\tau} c$.

3. If $c$ is moderated and $a *_{\tau} b \in \ell^{2}(\tau)$ then $a *_{\tau}\left(b *_{\tau} c\right)=\left(a *_{\tau} b\right) *_{\tau} c$.

Proof. Assume that $a$ is moderated and $b *_{\tau} c \in \ell^{2}(\tau)$. Then there is a sequence $a_{m} \in$ $\mathcal{F}^{\text {fin }}(S(I))$ such that $\left\|\lambda_{0}\left(a_{m}\right)\right\| \leq\left\|\lambda_{0}(a)\right\|$ and $\lambda_{0}\left(a_{m}\right) \rightarrow \lambda_{0}(a)$ in the weak operator topology. In view of the Mazur-Orlicz theorem we can assume that $\lambda_{0}\left(a_{m}\right)$ converges to $\lambda_{0}(a)$ in the strong operator topology. Therefore

$$
\left\|a_{m} *_{\tau}\left(b *_{\tau} c\right)-a *_{\tau}\left(b *_{\tau} c\right)\right\|_{2} \rightarrow 0,
$$

which implies the pointwise convergence. We have also $\left\|a_{m} *_{\tau} b-a *_{\tau} b\right\|_{2} \rightarrow 0$, which, by Lemma 2.2.1, implies $\left(\left(a_{m} *_{\tau} b\right) *_{\tau} c\right)(u) \rightarrow\left(\left(a *_{\tau} b\right) *_{\tau} c\right)(u)$ for every $u \in S(I)$. Since, by Lemma 2.2.2, $\left(a_{m} *_{\tau} b\right) *_{\tau} c=a_{m} *_{\tau}\left(b *_{\tau} c\right)$, we get the first statement. The third one can be proved in a similar way.

Now assume that $b$ is moderated. Similarly as before we can take a sequence $b_{n} \in$ $\mathcal{F}^{\text {fin }}(S(I))$ such that $\lambda_{0}\left(b_{n}\right) \rightarrow \lambda_{0}(b)$ in the strong operator topology. Appealing to the Mazur-Orlicz theorem again we can also assume that $\rho_{0}\left(b_{n}\right) \rightarrow \rho_{0}(b)$ in the strong operator topology. Therefore $\left\|a *_{\tau} b_{n}-a *_{\tau} b\right\|_{2} \rightarrow 0$ and $\left\|b_{n} *_{\tau} c-b *_{\tau} c\right\|_{2} \rightarrow 0$. Now we can conclude by applying Lemma 2.2 .

Proposition 2.19. Assume that $a, c \in \ell^{2}(\tau)$.

If $a$ is moderated then $\lambda_{0}(a) \rho_{0}(c) \subseteq \rho_{0}(c) \lambda_{0}(a)$ and $\lambda_{0}(a) \rho_{0}(c)^{*} \subseteq \rho_{0}(c)^{*} \lambda_{0}(a)$.

If $c$ is moderated then $\rho_{0}(c) \lambda_{0}(a) \subseteq \lambda_{0}(a) \rho_{0}(c)$ and $\rho_{0}(c) \lambda_{0}(a)^{*} \subseteq \lambda_{0}(a)^{*} \rho_{0}(c)$.

Proof. Suppose that $a$ is moderated and $b \in \mathcal{D}\left(\rho_{0}(c)\right)$. Then $\rho_{0}(c) b=b *_{\tau} c^{*}, \lambda_{0}(a) \rho_{0}(c) b=$ $a *_{\tau}\left(b *_{\tau} c^{*}\right)$ (Lemma 2.3) and, by definition of $\rho_{0}(c)$, there is a sequence $b_{n} \in \mathcal{F}^{\text {fin }}(S(I))$ such that $\left\|b_{n}-b\right\|_{2} \rightarrow 0$ and $\left\|b_{n} *_{\tau} c-b *_{\tau} c\right\|_{2} \rightarrow 0$. Take a sequence $a_{m} \in \mathcal{F}^{\text {fin }}(S(I))$ as in the proof of Proposition 2.18. Then

$$
a_{m} *_{\tau} b_{n} \rightarrow a_{m} *_{\tau} b
$$


as $n \rightarrow \infty$, in $\ell^{2}(\tau)$ for every $m$ and

$$
\left(a_{m} *_{\tau} b_{n}\right) *_{\tau} c=a_{m} *_{\tau}\left(b_{n} *_{\tau} c\right) \rightarrow a_{m} *_{\tau}\left(b *_{\tau} c\right)=\left(a_{m} *_{\tau} b\right) *_{\tau} c
$$

in $\ell^{2}(\tau)$, which means that $a_{m} *_{\tau} b \in \mathcal{D}\left(\rho_{0}(c)\right)$ and $\rho_{0}(c)\left(a_{m} *_{\tau} b\right)=\left(a_{m} *_{\tau} b\right) *_{\tau} c$ for every $m$. Since $\lambda_{0}\left(a_{m}\right) \rightarrow \lambda_{0}(a)$ in the strong operator topology we have $\left\|a_{m} *_{\tau} b-a *_{\tau} b\right\|_{2} \rightarrow 0$ and, by Proposition 2.18,

$$
\left(a_{m} *_{\tau} b\right) *_{\tau} c=a_{m} *_{\tau}\left(b *_{\tau} c\right) \rightarrow a *_{\tau}\left(b *_{\tau} c\right)=\left(a *_{\tau} b\right) *_{\tau} c
$$

in $\ell^{2}(\tau)$. Therefore $a *_{\tau} b \in \mathcal{D}\left(\rho_{0}(c)\right)$ and

$$
\rho_{0}(c) \lambda_{0}(a) b=\left(a *_{\tau} b\right) *_{\tau} c=a *_{\tau}\left(b *_{\tau} c\right)=\lambda_{0}(a) \rho_{0}(c) b .
$$

For the second inclusion we note that $\lambda_{0}(a)$, as an element of the von Neumann algebra $\mathcal{S}$, the weak closure of $\lambda_{0}(\mathcal{A}(\tau))$, can be expressed as $\lambda_{0}(a)=\sum_{k=1}^{4} \alpha_{k} U_{k}$, where $U_{k}$ are unitary elements of $\mathcal{S}$. Then $U_{k}=\lambda_{0}\left(u_{k}\right)$ for moderated $u_{k} \in \ell^{2}(\tau)$ and hence $U_{k} \rho_{0}(c) \subseteq \rho_{0}(c) U_{k}$. Assume that $f \in \mathcal{D}\left(\rho_{0}(c) U_{k}\right)$, which means that $g:=$ $U_{k} f \in \mathcal{D}\left(\rho_{0}(c)\right)$. Since $U_{k}^{-1}=\lambda_{0}\left(u_{k}^{*}\right)$ we also have $U_{k}^{-1} \rho_{0}(c) \subseteq \rho_{0}(c) U_{k}^{-1}$, which implies $U_{k}^{-1} g=f \in \mathcal{D}\left(\rho_{0}(c)\right)$. Therefore $U_{k} \rho_{0}(c)^{*}=\rho_{0}(c)^{*} U_{k}$. Multiplying both sides by $\alpha_{k}$ and taking the sum $\sum_{k=1}^{4}$ remains unchanged the domain on the left side, but can enlarge that on the right side.

\section{RePresentations WEAKLy CONTAined in THE REGUlAR ONE}

Let $\pi, \sigma$ be $*$-representations of a $*$-algebra $\mathcal{A}$. Then $\pi$ is said to be wekly contained in $\sigma$ if $\|\pi(a)\| \leq\|\sigma(a)\|$ holds for every $a \in \mathcal{A}$. This is equivalent to say that $\operatorname{ker}(\sigma) \subseteq$ $\operatorname{ker}(\pi)$, where $\operatorname{ker}(\pi)$ denotes the kernel of the extension of $\pi$ to the enveloping $C^{*}$ algebra of $\mathcal{A}$.

Following ideas of Haagerup [Ha] we are now going to describe those $*$-representations of $\mathcal{A}(\tau)$ which are weakly contained in the regular representation $\lambda_{0}$.

Theorem 3.1. Let $\left(\pi_{0}, \mathcal{H}_{0}\right)$ be a *-representation of $\mathcal{A}(\tau)$.

1. If, for every $\xi \in \mathcal{H}_{0}$ and $0<r<1$, the function

$$
S(I) \ni u \mapsto\left[\pi_{0}\left(\delta_{u}\right) \xi, \xi\right] \cdot r^{|u|}
$$

can be expressed as $f_{0} \cdot \tau$ for some $f_{0} \in \ell^{2}(\tau)$ then $\pi_{0}$ is weakly contained in $\lambda_{0}$.

2. Assume that there are constants $0<c_{1} \leq c_{2}$ such that $c_{1} \leq \tau_{i} \leq c_{2}$ for every $i \in I^{\text {fin }}$. If $\pi_{0}$ is weakly contained in the regular representation of $\mathcal{A}(\tau)$ then for every $\zeta, \eta \in \mathcal{H}_{0}$ and $0<r<1$ the function

$$
S(I) \ni u \mapsto\left[\pi_{0}\left(\delta_{u}\right) \zeta, \eta\right] \cdot r^{|u|}
$$

can be expressed as $f_{0} \cdot \tau$ for some $f_{0} \in \ell^{2}(\tau)$.

Set $E_{m}:=\{u \in S(I):|u|=m\}$ and let $\chi_{m}$ denote the characteristic function of $E_{m}$. First we prove two lemmas.

Lemma 3.2. Let $f$ and $g$ be two functions in $\mathcal{F}^{\mathrm{fin}}(S(I))$ with support in $E_{k}$ and $E_{l}$ respectively. Then

$$
\left\|\left(f *_{\tau} g\right) \cdot \chi_{m}\right\|_{2} \leq\|f\|_{2} \cdot\|g\|_{2}
$$

if $|k-l| \leq m \leq k+l$ and $k+l-m$ is even. Also,

$$
\left\|\left(f *_{\tau} g\right) \cdot \chi_{m}\right\|_{2} \leq \sqrt{C}\|f\|_{2} \cdot\|g\|_{2}
$$


if $|k-l| \leq m \leq k+l$ and $k+l-m$ is odd, where $C:=\sup \left\{\left|1-\tau_{i}\right|^{2} \cdot \tau_{i}^{-1}: i \in I^{\text {fin }}\right\}$. We have $\left\|\left(f *_{\tau} g\right) \cdot \chi_{m}\right\|_{2}=0$ for all other values of $m$.

Note that the constant $C$ is finite if and only if $\inf \left\{\tau_{i}: i \in I^{\mathrm{fin}}\right\}>0$ and $\sup \left\{\tau_{i}: i \in\right.$ $\left.I^{\text {fin }}\right\}<\infty$.

Proof. Writing $u v \in S(I)$ we will mean that the concatenation of $u$ and $v$ is in $S(I)$.

1) If $m=k+l$ then

$$
\begin{aligned}
\left\|\left(f *_{\tau} g\right) \cdot \chi_{m}\right\|_{2}^{2} & =\sum_{\substack{\left|u_{1}\right|=k,\left|u_{2}\right|=l \\
u_{1} u_{2} \in S(I)}}\left|f\left(u_{1}\right)\right|^{2} \cdot\left|g\left(u_{2}\right)\right|^{2} \tau\left(u_{1}\right) \tau\left(u_{2}\right) \\
& \leq \sum_{\substack{\left|u_{1}\right|=k,\left|u_{2}\right|=l \\
\mid}}\left|f\left(u_{1}\right)\right|^{2} \tau\left(u_{1}\right)\left|g\left(u_{2}\right)\right|^{2} \tau\left(u_{2}\right)=\|f\|_{2}^{2} \cdot\|g\|_{2}^{2} .
\end{aligned}
$$

2) Now assume that $m=k+l-2 p$ and define two auxiliary functions:

$$
f^{\prime}(w):=\left(\sum_{\substack{|v|=p, w v \in S(I)}}|f(w v)|^{2} \tau(v)\right)^{1 / 2}
$$

if $|w|=k-p$, and $f^{\prime}(w):=0$ otherwise,

$$
g^{\prime}(w):=\left(\sum_{\substack{|z|=p \\ z w \in S(I)}}|g(z w)|^{2} \tau(z)\right)^{1 / 2}
$$

if $|w|=l-p$, and $g^{\prime}(w):=0$ otherwise. Then we have

$$
\left\|f^{\prime}\right\|_{2}^{2}=\sum_{|w|=k-p}\left(\sum_{\substack{|v|=p, w v \in S(I)}}|f(w v)|^{2} \tau(v)\right) \tau(w)=\sum_{|z|=k}|f(z)|^{2} \tau(z)=\|f\|_{2}^{2}
$$

and similarly $\left\|g^{\prime}\right\|_{2}=\|g\|_{2}$.

Now fix $u=i_{1} \ldots i_{m}$ and put $u_{1}:=i_{1} \ldots i_{k-p}, u_{2}:=i_{k-p+1} \ldots i_{m}$. Using Cauchy inequality we get

$$
\left|\left(f *_{\tau} g\right)(u)\right| \leq \sum_{\substack{|v|=p, u_{1} v \in S(I), v^{*} u_{2} \in S(I)}}\left|f\left(u_{1} v\right) g\left(v^{*} u_{2}\right) \tau(v)\right| \leq f^{\prime}\left(u_{1}\right) g^{\prime}\left(u_{2}\right)=\left(f^{\prime} *_{\tau} g^{\prime}\right)(u) .
$$

Therefore, applying the previous point to $f^{\prime}$ and $g^{\prime}$,

$$
\left\|\left(f *_{\tau} g\right) \cdot \chi_{m}\right\|_{2} \leq\left\|\left(f^{\prime} *_{\tau} g^{\prime}\right) \cdot \chi_{m}\right\|_{2} \leq\left\|f^{\prime}\right\|_{2} \cdot\left\|g^{\prime}\right\|_{2}=\|f\|_{2} \cdot\|g\|_{2} .
$$


3) Assume that $m=k+l-1$. If $|u|=m$ then we can write $u=u_{1} i u_{2}$, with $\left|u_{1}\right|=k-1,\left|u_{2}\right|=l-1$ and we have $\left(f *_{\tau} g\right)(u)=f\left(u_{1} i\right) g\left(i u_{2}\right)\left(1-\tau_{i}\right)$. Therefore

$$
\begin{aligned}
\left\|\left(f *_{\tau} g\right) \cdot \chi_{m}\right\|_{2}^{2} & =\sum_{i_{1} \ldots i_{m} \in S(I)}\left|f\left(i_{1} \ldots i_{k}\right)\right|^{2}\left|g\left(i_{k} \ldots i_{m}\right)\right|^{2}\left(1-\tau_{i_{k}}\right)^{2} \tau\left(i_{1} \ldots i_{m}\right) \\
& =\sum_{i_{1} \ldots i_{m} \in S(I)}\left|f\left(i_{1} \ldots i_{k}\right)\right|^{2} \tau\left(i_{1} \ldots i_{k}\right)\left|g\left(i_{k} \ldots i_{m}\right)\right|^{2} \tau\left(i_{k} \ldots i_{m}\right) \frac{\left(1-\tau_{i_{k}}\right)^{2}}{\tau_{i_{k}}} \\
& \leq C\|f\|_{2}^{2} \cdot\|g\|_{2}^{2} .
\end{aligned}
$$

4) Finally let $m=k+l+1-2 p, p \geq 1$. Define

$$
f^{\prime}(w):=\left(\sum_{\substack{|v|=p \\ w v \in S(I)}}|f(w v)|^{2} \tau(v)\right)^{1 / 2}
$$

if $|w|=k+1-p$ and $f^{\prime}(w)=0$ otherwise, and similarly

$$
g^{\prime}(w):=\left(\sum_{\substack{|z|=p \\ z w \in S(I)}}|g(z w)|^{2} \tau(z)\right)^{1 / 2}
$$

if $|w|=l+1-p$ and $g^{\prime}(w)=0$ otherwise. Then we have

$$
\left\|f^{\prime}\right\|_{2}^{2}=\sum_{|w|=k+1-p}\left(\sum_{\substack{|v|=p \\ w v \in S(I)}}|f(w v)|^{2} \tau(v)\right) \tau(w)=\|f\|_{2}^{2}
$$

and similarly $\left\|g^{\prime}\right\|_{2}=\|g\|_{2}$.

Now fix $u=u_{1} i u_{2} \in S(I)$, with $\left|u_{1}\right|=k-p,\left|u_{2}\right|=l-p$. Then

$$
\begin{aligned}
& \left|\left(f *_{\tau} g\right)(u)\right| \leq \sum_{\substack{|v|=p \\
u_{i} i v \in S(I) \\
v^{*} i u_{2} \in S(I)}}\left|f\left(u_{1} i v\right) g\left(v^{*} i u_{2}\right) \tau(v)\left(1-\tau_{i}\right)\right| \\
& \quad \leq\left(\sum_{\substack{|v|=p \\
u_{1} i v \in S(I)}}\left|f\left(u_{1} i v\right)\right|^{2} \tau(v)\right)^{1 / 2}\left(\sum_{\substack{|v|=p \\
v^{*} i u_{2} \in S(I)}}\left|g\left(v^{*} i u_{2}\right)\right|^{2} \tau(v)\right)^{1 / 2}\left|1-\tau_{i}\right| \\
& =\left|f^{\prime}\left(u_{1} i\right) g^{\prime}\left(i u_{2}\right)\left(1-\tau_{i}\right)\right|=\left|\left(f^{\prime} *_{\tau} g^{\prime}\right)(u)\right| .
\end{aligned}
$$

Now from the previous point we get

$$
\left\|\left(f *_{\tau} g\right) \cdot \chi_{m}\right\|_{2} \leq\left\|\left(f^{\prime} *_{\tau} g^{\prime}\right) \cdot \chi_{m}\right\|_{2} \leq \sqrt{C}\|f\|_{2} \cdot\|g\|_{2} .
$$

Lemma 3.3. Let $f$ be a function supported on $E_{k}$. Then

$$
\left\|\lambda_{0}(f)\right\|_{2} \leq \sqrt{C+1}(2 k+1)\|f\|_{2},
$$

where, as before, $C=\sup \left\{\left|1-\tau_{i}\right|^{2} \cdot \tau_{i}^{-1}: i \in I^{\text {fin }}\right\}$. 
Proof. Fix $g \in \ell^{2}(\tau)$ and put $g_{l}:=g \cdot \chi_{l}$. Then, using the previous lemma and Cauchy's inequality, we have

$$
\begin{aligned}
\left\|\left(f *_{\tau} g\right) \cdot \chi_{m}\right\|_{2} & \leq \sum_{l=0}^{\infty}\left\|\left(f *_{\tau} g_{l}\right) \cdot \chi_{m}\right\|_{2} \\
& \leq\|f\|_{2}\left(\sum_{r=0}^{\min \{m, k\}}\left\|g_{m+k-2 r}\right\|_{2}+\sqrt{C} \sum_{r=1}^{\min \{m, k\}}\left\|g_{m+k+1-2 r}\right\|_{2}\right) \\
& \leq\|f\|_{2}\left(\sum_{r=0}^{2 \min \{m, k\}}\left\|g_{m+k-r}\right\|_{2}^{2}\right)^{1 / 2}\left(\sum_{r=0}^{\min \{m, k\}} 1+\sum_{r=1}^{\min \{m, k\}} C\right)^{1 / 2} \\
& \leq((k+1)(C+1))^{1 / 2}\|f\|_{2}\left(\sum_{r=0}^{2 \min \{m, k\}}\left\|g_{m+k-r}\right\|_{2}^{2}\right)^{1 / 2} .
\end{aligned}
$$

Therefore

$$
\begin{aligned}
\left\|f *_{\tau} g\right\|_{2}^{2} & \leq \sum_{m=0}^{\infty}\left\|\left(f *_{\tau} g\right) \cdot \chi_{m}\right\|_{2}^{2} \\
& \leq(C+1)(k+1)\|f\|_{2}^{2} \sum_{m=0}^{\infty}\left(\sum_{r=0}^{2 \min \{m, k\}}\left\|g_{m+k-r}\right\|_{2}^{2}\right) \\
& \leq(C+1)(k+1)\|f\|_{2}^{2} \cdot(2 k+1) \sum_{s=0}^{\infty}\left\|g_{s}\right\|_{2}^{2} \\
& =(C+1)(k+1)(2 k+1)\|f\|_{2}^{2} \cdot\|g\|_{2}^{2},
\end{aligned}
$$

and this concludes the proof.

Proof of Theorem 3.1. Suppose that for every $\xi \in \mathcal{H}_{0}$ and $0<r<1$ there is $f_{r} \in \ell^{2}(\tau)$ such that

$$
\phi_{r}(u):=\left[\pi_{0}\left(\delta_{u}\right) \xi, \xi\right] \cdot r^{|u|}=f_{r}(u) \cdot \tau(u)
$$

then, by Corollary 1.7 and $1.8, \phi_{r}$ is a $\tau$-positive definite function (as pointwise product of a $\tau$ - and a 0-positive definite function) and, by Theorem 2.14, is a coefficient of the regular representation $\lambda_{0}$. Therefore, letting $r \rightarrow 1$, we see that the function $u \mapsto$ $\left[\pi_{0}\left(\delta_{u}\right) \xi, \xi\right]$ is a pointwise limit of coefficients of $\lambda_{0}$ which proves that $\pi_{0}$ is weakly contained in $\lambda_{0}$.

Now assume that $\left(\pi_{0}, \mathcal{H}_{0}\right)$ is weakly contained in $\lambda_{0}$. Then in particular $\pi_{0}\left(\delta_{i}\right)=0$ whenever $i \in I \backslash I^{\mathrm{fin}}$, so we can assume that $I=I^{\mathrm{fin}}$. Fix $\zeta, \eta \in \mathcal{H}_{0}$ and put $g(u):=$ $\left[\pi_{0}\left(\delta_{u}\right) \zeta, \eta\right]$. For a function $f \in \ell^{2}(\tau)$ supported on $E_{k}$ we have

$$
\left[\pi_{0}(f) \zeta, \eta\right]=\sum_{|u|=k} f(u) g(u)=\sum_{|u|=k} f(u) \frac{g(u)}{\tau(u)} \tau(u),
$$

and, by Lemma 3.2,

$$
\left|\left[\pi_{0}(f) \zeta, \eta\right]\right| \leq\left\|\lambda_{0}(f)\right\|\|\zeta\|\|\eta\| \leq \sqrt{C+1}(2 k+1)\|f\|_{2}\|\zeta\|\|\eta\| .
$$


This means that

$$
\sum_{|u|=k}\left|\frac{g(u)}{\tau(u)}\right|^{2} \tau(u) \leq(C+1)(2 k+1)^{2}\|\zeta\|^{2}\|\eta\|^{2} .
$$

Hence, for every $0<r<1$ we have

$$
\sum_{u \in S(I)}\left|\frac{g(u)}{\tau(u)}\right|^{2} r^{2|u|} \tau(u)<\infty .
$$

\section{TyPE-DEPEndENT FUnCTIONS ON THE FREE PRODUCT GROUP}

Let $\left\{G_{i}\right\}_{i \in I}$ be a family of discrete nontrivial groups and let $G=*_{i \in I} G_{i}$ be their free product (see [Se]). Every element $x$ of $G$ can be uniquely represented as a reduced word:

$$
x=g_{1} g_{2} \ldots g_{m}, \quad m \geq 0, g_{k} \in G_{i_{k}} \backslash\{e\} \text { and } i_{k} \neq i_{k+1} \text { for } k<m .
$$

For such an element we define its length $|x|:=m$ and type as the formal word $t(x):=$ $i_{1} i_{2} \ldots i_{m} \in S(I)$. We are particularly interested in type-dependent functions on $G$, i.e. those which are compositions of the form $\phi \circ t$.

If all $G_{i}$ 's are finite then the family $\mathcal{F}_{t}(G)$ of finitely supported type dependent functions is isomorphic to $\mathcal{A}(\tau)$, with $\tau_{i}=\left(\left|G_{i}\right|-1\right)^{-1}$. Indeed, for $u \in S(I)$ put

$$
\mu_{u}(x):= \begin{cases}\tau(u) & \text { if } t(x)=u \\ 0 & \text { otherwise. }\end{cases}
$$

Then the map $f \mapsto \sum_{u \in S(I)} f(u) \mu_{u}$ is an isomorphism of $\mathcal{A}(\tau)$ onto $\mathcal{F}_{t}(G)$, which can be extended to an isometric embedding of $\ell^{2}(\tau)$ into $\ell^{2}(G)$.

Now we present an alternative proof of Theorem 3.2 in [M1], which characterizes the class of positive definite type-dependent functions.

Theorem 4.1. Let $\left\{G_{i}\right\}_{i \in I}$ be a family of groups, $G=*_{i \in I} G_{i}$, and let $\phi$ be a complex function on $S(I)$. The type-dependent function $\phi \circ t$ is positive definite on $G$ (resp. lies in the Fourier-Stielties algebra $B(G)$ ) if and only if $\phi$ is $\tau$-positive definite on $S(I)$ (resp. $\phi$ lies in $B(\tau))$, where $\tau_{i}=\left(\left|G_{i}\right|-1\right)^{-1}$.

If all $G_{i}$ 's are finite then the part concerning the Fourier-Stielties algebra could be derived from Theorem 3.2 and Corollary 3.3 in [M1].

Proof. During the proof we will regard $S(I)$ as a unital *-subgroup generated by elements $i \in I$ which satisfy $i i=i^{*}=i$.

Suppose that $\phi \circ t \in B(G)$, then $\phi \circ t$ can be represented as $\phi(t(x))=[\pi(x) \zeta, \eta]$ for a unitary representation $(\pi, \mathcal{H}$ ) of $G$ and vectors $\zeta, \eta \in \mathcal{H}$ (with $\zeta=\eta$ when $\phi \circ t$ is positive definite). We may assume that the vectors $\zeta$ and $\eta$ are both cyclic (cf. Proposition 1 in $[\mathrm{Sz}])$. If $G_{i}$ is finite then we put

$$
P_{i}=\frac{1}{\left|G_{i}\right|} \sum_{g \in G_{i}} \pi(g) \quad \text { and } \quad A_{i}=\frac{1}{\left|G_{i}\right|-1} \sum_{g \in G_{i} \backslash\{e\}} \pi(g) .
$$

Then we have $P_{i} P_{i}=P_{i}^{*}=P_{i}$ and $A_{i}=\left(1+\tau_{i}\right) P_{i}-\tau_{i} \mathrm{Id}$. 
Now, suppose that $G_{i}$ is infinite and let $\left\{g_{n, i}\right\}_{n=1}^{\infty}$ be a fixed sequence of distinct elements of $G_{i} \backslash\{e\}$. For any natural number $N$ we define an operator $T_{N, i}$ on $\mathcal{H}$ by

$$
T_{N, i}=\frac{1}{N} \sum_{n=1}^{N} \pi\left(g_{n, i}\right)
$$

For fixed $x, y \in G$ we have $t\left(y^{-1} g_{n, i} x\right)=t(y)^{*} i t(x)$ (product in the semigroup $S(I)$ ) for all but at most two $n$ 's. Therefore

$$
\begin{aligned}
{\left[T_{N, i} \pi(x) \zeta, \pi(y) \eta\right] } & =\frac{1}{N} \sum_{n=1}^{N}\left[\pi\left(y^{-1} g_{n, i} x\right) \zeta, \eta\right] \\
& =\frac{1}{N} \sum_{n=1}^{N} \phi\left(t\left(y^{-1} g_{n, i} x\right)\right) \rightarrow \phi\left(t(y)^{*} i t(x)\right)
\end{aligned}
$$

as $N \rightarrow \infty$. Since the vectors $\zeta, \eta$ are both cyclic and $T_{N, i}$ 's are all contractions, there exists a weak limit $A_{i}$ of the sequence $T_{N, i}$ satisfying

$$
\left[A_{i} \pi(x) \zeta, \pi(y) \eta\right]=\phi\left(t(y)^{*} i t(x)\right), \quad x, y \in G .
$$

Note that $A_{i}^{*}=A_{i}$. Indeed

$$
\lim _{N \rightarrow \infty}\left[T_{N, i}^{*} \pi(x) \zeta, \pi(y) \eta\right]=\lim _{N \rightarrow \infty} \frac{1}{N} \sum_{n=1}^{N} \phi\left(t\left(y^{-1} g_{n, i}^{-1} x\right)\right)=\phi\left(t(y)^{*} i t(x)\right) .
$$

Next we show that $A_{i} A_{i}=A_{i}$. Consider the operator $A_{i} T_{N, i}$. For any $x, y \in G$

$$
\begin{gathered}
{\left[A_{i} T_{N, i} \pi(x) \zeta, \pi(y) \eta\right]=\frac{1}{N} \sum_{n=1}^{N}\left[A_{i} \pi\left(g_{n, i} x\right) \zeta, \pi(y) \eta\right]} \\
=\frac{1}{N} \sum_{n=1}^{N} \phi\left(t(y)^{*} i t\left(g_{n, i} x\right)\right)=\phi\left(t(y)^{*} i t(x)\right)
\end{gathered}
$$

(because $i t(g x)=i t(x)$ if $\left.g \in G_{i}\right)$ hence $A_{i} T_{N, i}=A_{i}$, which implies $A_{i} A_{i}=A_{i}$. In this way we have defined the operator $A_{i}$ for every $i \in I$.

Now let $u=i_{1} i_{2} \ldots i_{m}$ be a fixed element in $S(I)$. For any $g_{2} \in G_{i_{2}} \backslash\{e\}, \ldots, g_{m} \in$ $G_{i_{m}} \backslash\{e\}$ we have

$$
\begin{aligned}
\phi(u) & =\left[\frac{1}{\left|G_{i_{1}}\right|-1} \sum_{g \in G_{i_{1} \backslash\{e\}}} \pi(g) \pi\left(g_{2} g_{3} \ldots g_{m}\right) \zeta, \eta\right] \\
& =\left[A_{i_{1}} \pi\left(g_{2} g_{3} \ldots g_{m}\right) \zeta, \eta\right]=\left[\pi\left(g_{2} g_{3} \ldots g_{m}\right) \zeta, A_{i_{1}} \eta\right]
\end{aligned}
$$

if $G_{i_{1}}$ is finite and

$$
\begin{aligned}
\phi(u) & =\left[T_{N, i_{1}} \pi\left(g_{2} g_{3} \ldots g_{m}\right) \zeta, \eta\right] \\
& =\left[A_{i_{1}} \pi\left(g_{2} g_{3} \ldots g_{m}\right) \zeta, \eta\right]=\left[\pi\left(g_{2} g_{3} \ldots g_{m}\right) \zeta, A_{i_{1}} \eta\right],
\end{aligned}
$$

for arbitrary $N$, if $G_{i_{1}}$ is infinite. Continuing in this fashion we finally obtain

$$
\phi(u)=\left[\zeta, A_{i_{m}} \ldots A_{i_{2}} A_{i_{1}} \eta\right]=\left[A_{i_{1}} A_{i_{2}} \ldots A_{i_{m}} \zeta, \eta\right]
$$

and, in view of Proposition 1.5, one part of the theorem is proved. 
Now assume that $\phi$ is of the form

$$
\phi\left(i_{1} i_{2} \ldots i_{m}\right)=\left[A_{i_{1}} A_{i_{2}} \ldots A_{i_{m}} \zeta, \eta\right],
$$

where $A_{i}=\left(1+\tau_{i}\right) P_{i}-\tau_{i} \mathrm{Id},\left\{P_{i}\right\}_{i \in I}$ is a family of orthogonal projections on a Hilbert space $\mathcal{H}_{0}$ and $\zeta, \eta \in \mathcal{H}_{0}$.

Fix $i \in I$. We show that the operator-valued function

$$
U_{i}(g)= \begin{cases}\text { Id } & \text { if } g=e, \\ A_{i} & \text { otherwise }\end{cases}
$$

is positive definite on the group $G_{i}$. Let $f: G_{i} \rightarrow \mathcal{H}_{0}$ be a finitely supported function. We can decompose $f$ as $f=f_{1}+f_{2}$ in such a way that $f_{1}: G_{i} \rightarrow \operatorname{Im} P_{i}, f_{2}: G_{i} \rightarrow \operatorname{Ker} P_{i}$. Then by the definition of $\tau_{i}$ we have

$$
\begin{aligned}
\sum_{g, h \in G_{i}} & {\left[U_{i}\left(h^{-1} g\right) f(g), f(h)\right] } \\
= & \sum_{g, h \in G_{i}}\left[f_{1}(g), f_{1}(h)\right]+\sum_{g \in G_{i}}\left[f_{2}(g), f_{2}(g)\right]-\tau_{i} \sum_{g \neq h}\left[f_{2}(g), f_{2}(h)\right] \\
= & \left\|\sum_{g \in G_{i}} f_{1}(g)\right\|^{2}+ \begin{cases}\sum_{g \in G_{i}}\left\|f_{2}(g)\right\|^{2} & \text { if } G_{i} \text { is infinite, } \\
\left(\tau_{i} / 2\right) \sum_{g \neq h}\left\|f_{2}(g)-f_{2}(h)\right\|^{2} & \text { otherwise. }\end{cases}
\end{aligned}
$$

Hence $U_{i}$ is positive definite on $G_{i}$. Now let us consider the function $U: G \rightarrow \mathcal{B}\left(\mathcal{H}_{0}\right)$ :

$$
U(x)=A_{i_{1}} A_{i_{2}} \ldots A_{i_{m}} \quad \text { if } \quad t(x)=i_{1} i_{2} \ldots i_{m} .
$$

By Theorem 7.1 in [B2] (which will be reproved in the next section) $U$ is positive definite on $G=*_{i \in I} G_{i}$, which implies that there exists a unitary representation $\pi: G \rightarrow \mathcal{B}(\mathcal{H})$ such that $\mathcal{H}_{0} \subseteq \mathcal{H}$ and for any $x \in G$ we have $U(x)=\left.P_{0} \pi(x)\right|_{\mathcal{H}_{0}}$, where $P_{0}$ denotes the orthogonal projection of $\mathcal{H}$ onto $\mathcal{H}_{0}$. This implies that $\phi(t(x))=[U(x) \zeta, \eta]=[\pi(x) \zeta, \eta]$, which concludes the proof.

\section{The INDUCED REPRESENTATION OF THE FREE PRODUCT GROUP}

Let $\mathcal{H}_{0}$ be a fixed Hilbert space, $G=*_{i \in I} G_{i}$ be the free product of groups and assume that for every $i \in I$ we are given a representation $\pi_{i}: G_{i} \rightarrow \mathcal{B}\left(\mathcal{H}_{0} \oplus \mathcal{H}_{i}\right.$ ) (we do not require that $\pi_{i}$ are unitary). We are going to construct a representation $\pi$ of $G$, acting on a Hilbert space $\mathcal{H}$ which contains all spaces $\mathcal{H}_{i}, i \in I \cup\{0\}$, such that for every $i \in I$ and $g \in G_{i}$ the restriction of $\pi(g)$ to $\mathcal{H}_{0} \oplus \mathcal{H}_{i}$ coincides with $\pi_{i}(g)$.

Construction of such kind were studied by Avitzour [Av], Voiculescu [Vo, VDN], another one was due to Bożejko [B1]. Then Bożejko, Leinert and Speicher [BS, BLS] generalized the previous ones by introducing conditionally free product of representations. In all these constructions the common subspace $\mathcal{H}_{0}$ was one-dimensional. Further generalizations can be found in [M3, M4, M5].

First we define for each $w \in G$ a Hilbert space $\mathcal{H}_{w}$ by putting

$$
\mathcal{H}_{e}=\mathcal{H}_{0} \oplus\left(\underset{i \in I}{\oplus} \mathcal{H}_{i}\right)
$$

and, for $w \neq e$,

$$
\mathcal{H}_{w}=\underset{j \in I \backslash\{i(w)\}}{\oplus} \mathcal{H}_{j}
$$


where for an element $x \neq e$ as in (4.1) we put $i(x):=i_{m}$, the type of the last letter of $x$. Now we define

$$
\mathcal{H}=\underset{w \in G}{\oplus} \mathcal{H}_{w}=\left\{f: G \rightarrow \mathcal{H}_{e} \mid f(w) \in \mathcal{H}_{w} \text { for } w \in G \text { and } \sum_{w \in G}\|f(w)\|^{2}<\infty\right\} .
$$

For every $w \in G$ and $\xi \in \mathcal{H}_{w}$ we denote by $(w, \xi)$ the function in $\mathcal{H}$ which has the value $\xi$ at $w$ and 0 elsewhere (i.e. $\left.(w, \xi):=\delta_{w} \otimes \xi\right)$. Then $\delta_{w} \otimes \mathcal{H}_{w}$ can be identified with the space of all functions in $\mathcal{H}$ vanishing outside $\{w\}$. We shall also identify $\mathcal{H}_{0}$ and $\mathcal{H}_{i}$ with the appropriate subspaces of $\delta_{e} \otimes \mathcal{H}_{e}$. If $i \in I \cup\{0\}$ then $Q_{i}$ will stand for the orthogonal projection of $\mathcal{H}_{e}$ onto $\mathcal{H}_{i}$.

Fix $i \in I$. First we define $\pi$ only on $G_{i}$ putting $\pi(e)=\operatorname{Id}$ and for $g \in G_{i} \backslash\{e\}$

$$
(\pi(g) f)(w)= \begin{cases}f\left(g^{-1}\right)+\pi_{i}(g)\left(Q_{0}+Q_{i}\right) f(e) & \text { if } w=e, \\ \left(\operatorname{Id}-Q_{0}-Q_{i}\right) f(e) & \text { if } w=g \\ f\left(g^{-1} w\right) & \text { otherwise }\end{cases}
$$

or, in terms of the vectors $(w, \xi)$,

$$
\pi(g)(w, \xi)= \begin{cases}\left(e, \pi_{i}(g)\left(Q_{0}+Q_{i}\right) \xi\right)+\left(g,\left(\mathrm{Id}-Q_{0}-Q_{i}\right) \xi\right) & \text { if } w=e, \\ g w, \xi) & \text { otherwise. }\end{cases}
$$

Then $\pi$ is a representation of $G_{i}$ which coincides with $\pi_{i}$ on $\mathcal{H}_{0} \oplus \mathcal{H}_{i}$ and is a multiple of the left regular representation on the orthogonal complement of $\mathcal{H}_{0} \oplus \mathcal{H}_{i}$. In particular, if $\pi_{i}$ is unitary then so is $\pi$.

Having defined $\pi(g)$ for all $g \in G_{i}$ and $i \in I$ we extend this to a representation all of $G$ by putting

$$
\pi(x)=\pi\left(g_{1}\right) \pi\left(g_{2}\right) \ldots \pi\left(g_{m}\right)
$$

for $x$ as in (4.1). Thus we obtained a representation $(\pi, \mathcal{H})$ of $G=*_{i \in I} G_{i}$ which we denote by $*_{i \in I} \pi_{i}$. Note that if all $\pi_{i}$ 's are unitary then so is $\pi$.

Lemma 5.1. Suppose that $x \neq e$ is as in (4.1) and $\xi \in \mathcal{H}_{e}$. Then

$$
\begin{gathered}
\pi(x)(e, \xi)=\left(e, \pi_{i_{1}}\left(g_{1}\right) Q_{0} \pi_{i_{2}}\left(g_{2}\right) Q_{0} \ldots Q_{0} \pi_{i_{m-1}}\left(g_{m-1}\right) Q_{0} \pi_{i_{m}}\left(g_{m}\right)\left(Q_{0}+Q_{i_{m}}\right) \xi\right) \\
+\sum_{k=1}^{m-1}\left(g_{1} g_{2} \ldots g_{k}, Q_{i_{k+1}} \pi_{i_{k+1}}\left(g_{k+1}\right) Q_{0} \pi_{i_{k+2}}\left(g_{k+2}\right) Q_{0} \ldots Q_{0} \pi_{i_{m}}\left(g_{m}\right)\left(Q_{0}+Q_{i_{m}}\right) \xi\right) \\
+\left(g_{1} g_{2} \ldots g_{m},\left(\mathrm{Id}-Q_{0}-Q_{i_{m}}\right) \xi\right) .
\end{gathered}
$$

Proof. We apply induction on the length of $x$. If $|x|=1$ then the formula is a consequence of the definition. Assume that it holds for $x$ as in (4.1) and take a word of the form $g_{0} x$, where $g_{0} \in G_{i_{0}} \backslash\{e\}, i_{0} \neq i_{1}$. Using the fact that if $\eta \in \mathcal{H}_{0} \oplus \mathcal{H}_{i_{1}}$ then $\left(Q_{0}+Q_{i_{0}}\right) \eta=Q_{0} \eta$ and $\left(\mathrm{Id}-Q_{0}-Q_{i_{0}}\right) \eta=Q_{i_{1}} \eta$ one can easily prove that the formula holds for $g_{0} x$.

Let us denote by $P_{0}$ the orthogonal projection of $\mathcal{H}$ onto $\mathcal{H}_{0}$.

Theorem 5.2. Suppose that $\pi=*_{i \in I} \pi_{i}$ is the representation of $G=*_{i \in I} G_{i}$ defined above. Then:

(i) If all $\pi_{i}$ 's are unitary then so is $\pi$.

(ii) If $\xi_{0} \in \mathcal{H}_{0}$ then, for $x$ as in (4.1),

$$
P_{0} \pi(x) \xi_{0}=Q_{0} \pi_{i_{1}}\left(g_{1}\right) Q_{0} \pi_{i_{2}}\left(g_{2}\right) \ldots Q_{0} \pi_{i_{m}}\left(g_{m}\right) \xi_{0} .
$$


(iii) Assume that there are constants $a_{1}, a_{2}, \ldots$ such that

$$
\left\|\pi_{j_{1}}\left(h_{1}\right) Q_{0} \pi_{j_{2}}\left(h_{2}\right) \ldots Q_{0} \pi_{j_{n}}\left(h_{n}\right)\right\| \leq a_{n}
$$

(the operator norm of a map $\mathcal{H}_{0} \oplus \mathcal{H}_{j_{n}} \rightarrow \mathcal{H}_{0} \oplus \mathcal{H}_{j_{1}}$ ) holds for every $j_{1} j_{2} \ldots j_{n} \in S(I)$ and every $h_{1} \in G_{j_{1}} \backslash\{e\}, \ldots, h_{n} \in G_{j_{n}} \backslash\{e\}$. Then for every $x \in G$

$$
\|\pi(x)\| \leq 1+a_{1}+\cdots+a_{m}
$$

where $m=|x|$. In particular, if $\sum a_{n}<\infty$ then $\pi$ is uniformly bounded.

(iv) Assume that for each $i \in I$ the set $\left\{\pi_{i}(g) \xi: g \in G_{i}, \xi \in \mathcal{H}_{0}\right\}$ is linearly dense in $\mathcal{H}_{0} \oplus \mathcal{H}_{i}$. Then the family $\left\{\pi(x)(e, \xi): x \in G, \xi \in \mathcal{H}_{0}\right\}$ is linearly dense in $\mathcal{H}$.

Proof. We have already noticed that unitarity of $\pi_{i}$ 's implies that of $\pi$ and part (ii) is a consequence of the last lemma. Now we will prove (iii).

Fix $x=g_{1} g_{2} \ldots g_{m} \neq e$ as in (4.1). For $1 \leq r \leq m$ define $w_{r}=\left(g_{r+1} g_{r+2} \ldots g_{m}\right)^{-1}$. By the previous lemma we have

$$
\begin{aligned}
\pi(x)\left(w_{r}, \xi\right)= & \pi\left(g_{1} g_{2} \ldots g_{r}\right)(e, \xi) \\
= & \left(e, \pi_{i_{1}}\left(g_{1}\right) Q_{0} \pi_{i_{2}}\left(g_{2}\right) Q_{0} \ldots Q_{0} \pi_{i_{r-1}}\left(g_{r-1}\right) Q_{0} \pi_{i_{r}}\left(g_{r}\right)\left(Q_{0}+Q_{i_{r}}\right) \xi\right) \\
& +\sum_{k=1}^{r-1}\left(g_{1} g_{2} \ldots g_{k}, Q_{i_{k+1}} \pi_{i_{k+1}}\left(g_{k+1}\right) Q_{0} \pi_{i_{k+2}} Q_{0} \ldots Q_{0} \pi_{i_{r}}\left(g_{r}\right)\left(Q_{0}+Q_{i_{r}}\right) \xi\right) \\
& +\left(g_{1} g_{2} \ldots g_{r},\left(\mathrm{Id}-Q_{0}-Q_{i_{r}}\right) \xi\right) .
\end{aligned}
$$

On the other hand if $w$ is none of $w_{r}, 1 \leq r \leq m$, then $\pi(x)(w, \xi)=(x w, \xi)$.

Consider the following operators on $\mathcal{H}$ :

$$
T_{0}(w, \xi)= \begin{cases}\left(x w_{r},\left(\mathrm{Id}-Q_{0}-Q_{i_{r}}\right) \xi\right) & \text { if } w=w_{r}, 1 \leq r \leq m \\ (x w, \xi) & \text { otherwise }\end{cases}
$$

and for $1 \leq s \leq m$ we define $T_{s}$ putting

$$
\begin{aligned}
& T_{s}\left(w_{s}, \xi\right)=\left(e, \pi_{i_{1}}\left(g_{1}\right) Q_{0} \pi_{i_{2}}\left(g_{2}\right) Q_{0} \ldots Q_{0} \pi_{i_{s}}\left(g_{s}\right)\left(Q_{0}+Q_{i_{s}}\right) \xi\right) \\
& T_{s}\left(w_{r}, \xi\right)=\left(g_{1} \ldots g_{r-s}, Q_{i_{r-s+1}} \pi_{i_{r-s+1}}\left(g_{r-s+1}\right) Q_{0} \ldots Q_{0} \pi_{i_{r}}\left(g_{r}\right)\left(Q_{0}+Q_{i_{r}}\right) \xi\right)
\end{aligned}
$$

if $s<r \leq m$, and $T_{s}(w, \xi)=0$ if $w$ is not one of $w_{r}$ for $s \leq r \leq m$. Then, putting $a_{0}=1$, we have $\left\|T_{s}\right\| \leq a_{s}$ and $\pi(x)=T_{0}+T_{1}+\cdots+T_{m}$, which proves (iii).

To prove (iv) denote by $M$ the closure of the linear hull of the set $\{\pi(x)(e, \xi): x \in$ $\left.G, \xi \in \mathcal{H}_{0}\right\}$. Then $M$ is $G$-invariant and $\mathcal{H}_{0} \subseteq M$. By the assumption, $\delta_{e} \otimes \mathcal{H}_{i} \subseteq M$ for every $i \in I$. Therefore $\delta_{x} \otimes \mathcal{H}_{i}=\pi(x)\left(\delta_{e} \otimes \mathcal{H}_{i}\right) \subseteq M$ for every $x \in G$ and $i \neq i(x)$, if $x \neq e$. But the family of such subspaces is linearly dense in $\mathcal{H}$, which concludes the proof.

As a corollary we will reprove Bożejko's result (Theorem 7.1 in [B2]).

Corollary 5.3. Let $\mathcal{H}_{0}$ be a Hilbert space and assume that for each $i \in I$ we have an operator-valued positive definite function $U_{i}: G_{i} \rightarrow \mathcal{B}\left(\mathcal{H}_{0}\right)$ satisfying $U_{i}(e)=\mathrm{Id}$. Define $U: G \rightarrow \mathcal{B}\left(\mathcal{H}_{0}\right)$ putting

$$
U(x)=U_{i_{1}}\left(g_{1}\right) U_{i_{2}}\left(g_{2}\right) \ldots U_{i_{m}}\left(g_{m}\right)
$$

for $x=g_{1} g_{2} \ldots g_{m}$ as in (4.1). Then $U$ is a positive definite function on $G$. 
Proof. By [NF], Theorem 7.1 for each $i \in I$ there is a Hilbert space $\mathcal{H}_{i}$ and a unitary representation $\pi_{i}: G_{i} \rightarrow \mathcal{B}\left(\mathcal{H}_{0} \oplus \mathcal{H}_{i}\right)$ such that if $\xi \in \mathcal{H}_{0}, g \in G_{i}$ then $U_{i}(g) \xi=Q_{0} \pi_{i}(g) \xi$, where $Q_{0}$ denotes the orthogonal projection of $\mathcal{H}_{0} \oplus \mathcal{H}_{i}$ onto $\mathcal{H}_{0}$. Take the representation $\pi=*_{i \in I} \pi_{i}$ of $G$ constructed as above. Then $\pi$ is unitary and if $\xi \in \mathcal{H}_{0}, x \in G$ then $P_{0} \pi(x) \xi=U(x) \xi$ which implies positive definiteness of $U$.

Now assume that $\left\{P_{i}\right\}_{i \in I}$ is a family of (not necessarily orthogonal) projections on a Hilbert space $\mathcal{H}_{0}$. With every $i \in I$ we associate a space $\mathcal{H}_{i}$ and a representation $\pi_{i}: G_{i} \rightarrow \mathcal{B}\left(\mathcal{H}_{0} \oplus \mathcal{H}_{i}\right)$ in the following way.

First assume that $G_{i}$ is infinite. Then we set

$$
\mathcal{H}_{i}:=\left\{f: G_{i} \backslash\{e\} \rightarrow \operatorname{Ker} P_{i} \mid \sum_{g \in G_{i} \backslash\{e\}}\|f(g)\|^{2}<\infty\right\},
$$

so that

$$
\mathcal{H}_{0} \oplus \mathcal{H}_{i}=\left\{f: G_{i} \rightarrow \mathcal{H}_{0} \mid f(g) \in \operatorname{Ker} P_{i} \text { for } g \neq e \text { and } \sum_{g \in G_{i}}\|f(g)\|^{2}<\infty\right\} .
$$

Now we define $\pi_{i}(e)=\mathrm{Id}$, and for $g \in G_{i} \backslash\{e\}$

$$
\left(\pi_{i}(g) f\right)(h)= \begin{cases}P_{i} f(e)+f\left(g^{-1}\right) & \text { if } h=e \\ \left(\mathrm{Id}-P_{i}\right) f(e) & \text { if } h=g \\ f\left(g^{-1} h\right) & \text { otherwise. }\end{cases}
$$

Note that $\pi_{i}$ acts trivially on $\operatorname{Im} P_{i}$ and as a multiple of the regular representation $\lambda_{i}$ of $G_{i}$ on $\operatorname{Ker} P_{i} \oplus \mathcal{H}_{i}$.

The case when $G_{i}$ is finite is a little bit more involved. Put

$$
\mathcal{H}_{i}=\left\{f: G_{i} \backslash\{e\} \rightarrow \operatorname{Ker} P_{i} \mid \sum_{g \in G_{i} \backslash\{e\}} f(g)=0\right\}
$$

so that

$$
\mathcal{H}_{0} \oplus \mathcal{H}_{i}=\left\{f: G_{i} \rightarrow \mathcal{H}_{0} \mid \text { if } g \neq e \text { then } f(g) \in \operatorname{Ker} P_{i} \text { and } \sum_{g \in G_{i} \backslash\{e\}} f(g)=0\right\} .
$$

Now we note that $\operatorname{Ker} P_{i} \oplus \mathcal{H}_{i}$ can be identified with

$$
\mathcal{H}_{i}^{\prime}:=\left\{F: G_{i} \rightarrow \operatorname{Ker} P_{i} \mid \sum_{g \in G_{i}} F(g)=0\right\}
$$

by an isometry $T_{i}: \mathcal{H}_{i}^{\prime} \rightarrow \operatorname{Ker} P_{i} \oplus \mathcal{H}_{i}$ :

$$
T_{i} F(h)= \begin{cases}\sqrt{1+\tau_{i}} \cdot F(e) & \text { if } h=e, \\ F(h)+\tau_{i} \cdot F(e) & \text { otherwise }\end{cases}
$$

where $\tau_{i}=\left(\left|G_{i}\right|-1\right)^{-1}$. Observe that $T_{i}^{-1}\left(\operatorname{Ker} P_{i}\right)$ consists of functions of the form $e \mapsto \xi, g \mapsto-\tau_{i} \cdot \xi$ for $g \neq e$, with $\xi \in \operatorname{Ker} P_{i}$. The natural representation $\pi_{i}^{\prime}$ of $G_{i}$ acting on $\mathcal{H}_{i}^{\prime}$ is a multiple of the semiregular representation:

$$
\left(\pi_{i}^{\prime}(g) F\right)(h):=F\left(g^{-1} h\right) .
$$

Now we define a representation $\pi_{i}: G_{i} \rightarrow \mathcal{B}\left(\mathcal{H}_{0} \oplus \mathcal{H}_{i}\right)$ putting

$$
\left(\pi_{i}(g) f\right):=\widetilde{P}_{i} f+\left(T_{i} \pi_{i}^{\prime}(g) T_{i}^{-1}\right)\left(\mathrm{Id}-\widetilde{P}_{i}\right) f,
$$


where $\widetilde{P}_{i}$ is a projection on $\mathcal{H}_{0} \oplus \mathcal{H}_{i}$ given by

$$
\widetilde{P}_{i} f(h)= \begin{cases}P_{i} f(e) & \text { if } h=e, \\ 0 & \text { otherwise. }\end{cases}
$$

As a result, for $g \in G_{i} \backslash\{e\}$ we obtain the following formula

$$
\left(\pi_{i}(g) f\right)(h)= \begin{cases}P_{i} f(e)+\sqrt{1+\tau_{i}} \cdot f\left(g^{-1}\right)-\tau_{i} \cdot\left(\operatorname{Id}-P_{i}\right) f(e) & \text { if } h=e, \\ \tau_{i} \cdot f\left(g^{-1}\right)+\left(1-\tau_{i}\right) \sqrt{1+\tau_{i}} \cdot\left(\operatorname{Id}-P_{i}\right) f(e) & \text { if } h=g, \\ f\left(g^{-1} h\right)+\tau_{i} \cdot f\left(g^{-1}\right)-\tau_{i} \sqrt{1+\tau_{i}} \cdot\left(\operatorname{Id}-P_{i}\right) f(e) & \text { otherwise }\end{cases}
$$

Now we are going to define the main object of this paper.

Definition 5.4. Assume that $\left\{G_{i}\right\}_{i \in I}$ is a family of discrete groups and put $\tau_{i}:=$ $\left(\left|G_{i}\right|-1\right)^{-1}$. Let $\pi_{0}: \mathcal{A}(\tau) \rightarrow \mathcal{B}\left(\mathcal{H}_{0}\right)$ be a representation of $\mathcal{A}(\tau)$ (not necessarily a *-representation) so that $\pi_{0}\left(\delta_{i}\right)=\left(1+\tau_{i}\right) P_{i}-\tau_{i}$ Id for a (not necessarily orthogonal) projection $P_{i}$ on $\mathcal{H}_{0}$. For every $i \in I$ define a representation $\pi_{i}$ of $G_{i}$ by (5.2) and then define a representation $\pi$ of $G:=*_{i \in I} G_{i}$ using formulas (5.1). Then we will say that $\pi$ is induced from the representation $\pi_{0}$ of $\mathcal{A}(\tau)$.

Now we observe that $\pi$ inherits some properties of $\pi_{0}$.

Theorem 5.5. Assume that $G=*_{i \in I} G_{i}, \tau_{i}=\left(\left|G_{i}\right|-1\right)^{-1}, \pi_{0}: \mathcal{A}(\tau) \rightarrow \mathcal{B}\left(\mathcal{H}_{0}\right)$ is a representation and let $\pi: G \rightarrow \mathcal{B}(\mathcal{H})$ be the induced representation of $G$.

i. For every $x \in G$ we have $\left.P_{0} \pi(x)\right|_{\mathcal{H}_{0}}=\pi_{0}\left(\delta_{t(x)}\right)$.

ii. If $\mathcal{H}_{0}$ can be decomposed into direct sum $\mathcal{H}_{0}^{\prime}+\mathcal{H}_{0}^{\prime \prime}$ of $\mathcal{A}(\tau)$-invariant closed subspaces then $\mathcal{H}$ can be decomposed into direct sum $\mathcal{H}^{\prime}+\mathcal{H}^{\prime \prime}$ of $G$-invariant closed subspaces, and the corresponding representations $\pi^{\prime}, \pi^{\prime \prime}$ of $G$ are induced from the corresponding representations $\pi_{0}^{\prime}, \pi_{0}^{\prime \prime}$ of $\mathcal{A}(\tau)$. If the former sum is orthogonal then so is the latter.

iii. If $\pi_{0}$ is the left regular representation of $\mathcal{A}(\tau)$ then $\pi$ is the left regular representation of $G$.

iv. If $\pi_{0}$ is a *-representation then $\pi$ is a unitary representation of $G$.

v. If there are constants $d_{m}$ such that $\left\|\pi_{0}\left(\delta_{u}\right)\right\| \leq d_{|u|}$ for every $u \in S(I)$ and if $\sum_{m=0}^{\infty} d_{m}<\infty$ then $\pi$ is uniformly bounded.

vi. The set $\left\{\pi(x)(e, \xi): x \in G, \xi \in \mathcal{H}_{0}\right\}$ is linearly dense in $\mathcal{H}$.

Proof. (i) By formula (5.2) and Theorem 5.2.ii we have $\left.P_{0} \pi(x)\right|_{\mathcal{H}_{0}}=B_{i_{1}} \ldots B_{i_{m}}$, where $B_{i}=\left(1+\tau_{i}\right) P_{i}-\tau_{i} \mathrm{Id}$, so $\left.P_{0} \pi(x)\right|_{\mathcal{H}_{0}}=\pi_{0}\left(\delta_{t(x)}\right)$.

(ii) Let $P$ be the projection of $\mathcal{H}_{0}$ onto $\mathcal{H}_{0}^{\prime}$ with $\operatorname{ker} P=\mathcal{H}_{0}^{\prime \prime}$. Replacing $\operatorname{ker} P_{i}$ by $P \operatorname{ker} P_{i}$ and $(\mathrm{Id}-P) \operatorname{ker} P_{i}$ in the definition of $\mathcal{H}_{i}$ we decompose $\mathcal{H}_{i}$ into direct sum $\mathcal{H}_{i}^{\prime}+\mathcal{H}_{i}^{\prime \prime}$ of $G_{i}$-invariant subspaces, which leads to the decomposition of all of $\mathcal{H}$ into a direct sum $\mathcal{H}^{\prime}+\mathcal{H}^{\prime \prime}$ of $G$-invariant subspaces.

To prove (iii) we note that the vector $\delta_{e}$ is cyclic for $\lambda_{0}$ and $\left[\lambda_{0}(a) \delta_{e}, \delta_{e}\right]=a(e)$ for $a \in \mathcal{A}(\tau)$. In view of the previous point we have

$$
\left[\pi(x) \delta_{e}, \delta_{e}\right]=\left[P_{0} \pi(x) \delta_{e}, \delta_{e}\right]=\left[\pi_{0}\left(\delta_{t(x)}\right) \delta_{e}, \delta_{e}\right]= \begin{cases}1 & \text { if } x=e, \\ 0 & \text { otherwise }\end{cases}
$$

for $x \in G$, and, since the vectors $\pi(x) \xi, x \in G, \xi \in \mathcal{H}_{0}$, are linearly dense in $\mathcal{H}$, the vector $\delta_{e}$ is cyclic for $\pi$. It means that $\pi$ is the left regular representation of $G$.

If $\pi_{0}$ is a $*$-representation then all the projections $P_{i}$ are orthogonal and then every representation $\pi_{i}$ of $G_{i}$ is unitary, which implies unitarity of $\pi$. 
Assume that $\left\|\pi_{0}\left(\delta_{u}\right)\right\| \leq d_{|u|}$ for every $u \in S(I)$. Then the norms $\left\|P_{i}\right\|$ are uniformly bounded, which implies that there is a constant $C$ such that $\left\|\pi_{i}(g)\right\| \leq C$ for every $i \in I$ and $g \in G_{i}$. Then for $y=h_{1} \ldots h_{n}$, with $t(y)=j_{1} \ldots j_{n}$, we have

$$
\begin{gathered}
\left\|\pi_{j_{1}}\left(h_{1}\right) Q_{0} \ldots Q_{0} \pi_{j_{n}}\left(h_{n}\right)\right\| \\
=\left\|\pi_{j_{1}}\left(h_{1}\right) B_{j_{2}} B_{j_{3}} \ldots B_{j_{n-1}} \pi_{j_{n}}\left(h_{n}\right)\right\| \leq C^{2}\left\|B_{j_{2}} \ldots B_{j_{n-1}}\right\| \leq C^{2} d_{n-2},
\end{gathered}
$$

and in view of Theorem 5.2 iii we see that $\pi$ is uniformly bounded.

For (vi) it suffices, by Theorem 5.2.iv, to prove that for every $i \in I$ the set $\left\{\pi_{i}(g) \xi\right.$ : $\left.g \in G_{i}, \xi \in \mathcal{H}_{0}\right\}$ is linearly dense in $\mathcal{H}_{0} \oplus \mathcal{H}_{i}$. If $G_{i}$ is infinite then it is clear that the linear span of vectors of the form $\delta_{g} \otimes \xi=\pi_{i}(g) \xi$, where $g \in G_{i} \backslash\{e\}, \xi \in \operatorname{Ker} P_{i}$, is dense in $\mathcal{H}_{i}$.

Now, if $G_{i}$ is finite then it is sufficient to check, that

$$
\left\{\pi_{i}^{\prime}(g) F: g \in G_{i}, F \in T_{i}^{-1}\left(\operatorname{Ker} P_{i}\right)\right\}=\mathcal{H}_{i}^{\prime} .
$$

Fix $F \in \mathcal{H}_{i}^{\prime}$. For $g \in G_{i}$ we define $F_{g} \in T_{i}^{-1}\left(\operatorname{Ker} P_{i}\right)$ by putting

$$
F_{g}(h):= \begin{cases}\frac{1}{1+\tau_{i}} F(g) & \text { if } h=e, \\ \frac{-\tau_{i}}{1+\tau_{i}} F(g) & \text { if } h \neq e .\end{cases}
$$

Then one can check that $\sum_{g \in G_{i}} \pi_{i}^{\prime}(g) F_{g}=f$, which concludes the proof.

For further investigations we will need two lemmas.

Lemma 5.6. Given a *-representation $\rho$ of a *-algebra $\mathcal{A}$ acting on a Hilbert space $\mathcal{H}$ and a family $\left\{\rho_{\alpha}\right\}, \alpha \in A$, of subrepresentations of $\rho$, each $\rho_{\alpha}$ acting on $\mathcal{H}_{\alpha} \subseteq \mathcal{H}$, such that the set $\bigcup_{\alpha \in A} \mathcal{H}_{\alpha}$ is linearly dense in $\mathcal{H}$, then $\rho$ is equivalent to a subrepresentation of the direct sum $\oplus_{\alpha \in A} \rho_{\alpha}$.

Proof. We may assume that the index set $A$ is an ordinal. Put

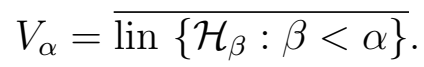

Fix $\alpha$ and let $W_{\alpha}$ be the orthogonal complement of $V_{\alpha}$ in $V_{\alpha+1}$. Consider $P: \mathcal{H}_{\alpha} \mapsto W_{\alpha}$ being the orthogonal projection. Take the polar decomposition $P=U S$, where $S$ is positive definite and $U$ is a partial isometry. By definition of $V_{\alpha+1}$ the image of $\mathcal{H}_{\alpha}$ is dense in $W_{\alpha}$ so $U^{-1}$ is an isometric embedding of $W_{\alpha}$ in $\mathcal{H}_{\alpha}$. Moreover, by the uniqueness of polar decomposition $U^{-1}$ intertwines the action of $\mathcal{A}$ on $W_{\alpha}$ with the action of $\mathcal{A}$ on $\mathcal{H}_{\alpha}$. Let $\widetilde{\rho}_{\alpha}$ be the restriction of $\rho$ to $W_{\alpha}$. By definition

$$
\oplus_{\alpha \in A} W_{\alpha}=\overline{\operatorname{lin}\left\{\mathcal{H}_{\beta}: \beta \in A\right\}}=\mathcal{H}
$$

so $\oplus_{\alpha \in A} \widetilde{\rho}_{\alpha}=\rho$. Since each $\widetilde{\rho}_{\alpha}$ is equivalent to a subrepresentation of $\rho_{\alpha}$ the claim follows.

Having constructed the induced representation $\pi$ we can in turn define a representation of $\mathcal{A}(\tau)$ acting on $\mathcal{H}$. Namely we put

$$
\tilde{\pi}_{0}\left(\delta_{i}\right)= \begin{cases}\tau_{i} \cdot \sum_{g \in G_{i} \backslash\{e\}} \pi(g) & \text { if } G_{i} \text { is finite, } \\ \lim \frac{1}{n} \sum_{k=1}^{n} \pi\left(g_{k, i}\right) & \text { otherwise, }\end{cases}
$$

where $g_{k, i}$ is an arbitrary sequence of distinct elements of $G_{i}$ and the limit is in the strong operator topology. Note that $\widetilde{\pi}_{0}$ restricted to $\mathcal{H}_{0}$ is just $\pi_{0}$ and that if $G_{i}$ is infinite then $\widetilde{\pi}_{0}\left(\delta_{i}\right)=\pi_{0}\left(\delta_{i}\right) P_{0}$, where $P_{0}$ is the orthogonal projection of $\mathcal{H}$ onto $\mathcal{H}_{0}$. 
Lemma 5.7. The restriction of $\tilde{\pi}_{0}$ to $\mathcal{H}_{0}^{\perp}$ is contained in a multiple of the left regular representation.

Proof. For $i \in I, \xi \in \mathcal{H}_{i}$ we have $\pi\left(\mu_{i}\right)(e, \xi)=-\tau_{i}(e, \xi)$ and for a vector $(w, \xi)$ in $\left(\mathcal{H}_{0} \oplus \mathcal{H}_{i}\right)^{\perp}$ we have $\pi\left(\mu_{i}\right)(w, \xi)=\tau_{i} \sum_{g \in G_{i} \backslash\{e\}}(g w, \xi)$. Therefore for every $w \in G$ and $(w, \xi) \in \mathcal{H}_{0}^{\perp}$ the $\tau$-positive definite function

$$
u \mapsto\left[\pi\left(\mu_{u}\right)(w, \xi),(w, \xi)\right]
$$

on $S(I)$ has a finite support and hence belongs to $\ell^{2}(\tau)$.

Here we give one application of the induced representation. Recall that for a $*$-algebra $\mathcal{A}$ the enveloping $C^{*}$-algebra $C^{*}(\mathcal{A})$ is defined as the completion of $\mathcal{A}$ with respect to the norm

$$
\|a\|:=\sup \{\|\pi(a)\|: \pi \text { is a } * \text {-representation of } \mathcal{A}\} .
$$

If $\Gamma$ is a discrete group then $C^{*}(\mathcal{F}(\Gamma))$ is called the full $C^{*}$-algebra of $\Gamma$ and denoted $C^{*}(\Gamma)$. Define also the reduced $C^{*}$-algebra of $\Gamma$ (resp. of the algebra $\mathcal{A}(\tau)$ ), denoted $C_{r}^{*}(\Gamma)\left(\right.$ or $C_{r}^{*}(\mathcal{A}(\tau))$ ), as the closure of $\mathcal{F}(\Gamma)$ (resp. of $\left.\mathcal{F}(S(I))\right)$ in the operator norm $\|\lambda(f)\|$ (resp. $\left.\left\|\lambda_{0}(a)\right\|\right)$.

Proposition 5.8. Suppose that all the groups $G_{i}$ are finite, $\tau_{i}=\left(\left|G_{i}\right|-1\right)^{-1}$ and $G=*_{i \in I} G_{i}$. Then the map

$$
\mathcal{A}(\tau) \ni a \mapsto \sum_{u \in S(I)} a(u) \mu_{u} \in \mathcal{F}(G)
$$

extends to an isometric embedding of $C^{*}(\mathcal{A}(\tau))$ into $C^{*}(G)$ and to an isometric embedding of $C_{r}^{*}(\mathcal{A}(\tau))$ into $C_{r}^{*}(G)$.

Proof. Put $j(a):=\sum_{u \in S(I)} a(u) \mu_{u}$. If $\pi$ is a unitary representation of $G$ then $\pi \circ j$ is a *-representation of $\mathcal{A}(\tau)$, which implies that $\|j(a)\| \leq\|a\|$ for $a \in \mathcal{A}(\tau)$. On the other hand, if $\pi_{0}$ is a $*$-representation of $\mathcal{A}(\tau)$ then taking the induced representation $\pi$ of $G$ we have $\left\|\pi_{0}(a)\right\| \leq\|\pi(j(a))\|$, which yields $\|a\| \leq\|j(a)\|$.

The second statement holds because the map $a \mapsto \lambda(j(a))$ is a multiple of the left regular representation of $\mathcal{A}(\tau)$.

Recall that if $\pi: \mathcal{A} \rightarrow \mathcal{B}(\mathcal{H})$ and $\sigma: \mathcal{A} \rightarrow \mathcal{B}(\mathcal{K})$ are representations of an algebra $\mathcal{A}$ then $T: \mathcal{H} \rightarrow \mathcal{K}$ is said to be an intertwining operator if $T \pi(a)=\sigma(a) T$ holds for every $a \in \mathcal{A}$. Representations $\pi$ and $\sigma$ are called equivalent if there is an intertwining isomorphism $T: \mathcal{H} \rightarrow \mathcal{K}$. Representations $\pi$ and $\sigma$ are said to be disjoint if 0 is the only operator intertwining them.

Lemma 5.9. Let $\mathcal{A}_{0}$ be a subalgebra of an algebra $\mathcal{A}$ and let $\rho: \mathcal{A} \rightarrow \mathcal{B}(\mathcal{H}), \sigma: \mathcal{A} \rightarrow$ $\mathcal{B}(\mathcal{K})$ be representations. Denote by $\rho_{0}$ and $\sigma_{0}$ their restrictions to $\mathcal{A}_{0}$. We assume that $\mathcal{H}=\mathcal{H}_{0} \oplus \mathcal{H}_{1}, \mathcal{K}=\mathcal{K}_{0} \oplus \mathcal{K}_{1}$ are decompositions into direct sums of $\mathcal{A}_{0}$-invariant closed subspaces.

1. If $\left.\rho_{0}\right|_{\mathcal{H}_{0}}$ is disjoint from $\left.\sigma_{0}\right|_{\mathcal{K}_{1}},\left.\rho_{0}\right|_{\mathcal{H}_{1}}$ is disjoint from $\left.\sigma_{0}\right|_{\mathcal{K}_{0}}$ and $T: \mathcal{H} \rightarrow \mathcal{K}$ intertwines $\rho$ and $\sigma$ then $T \mathcal{H}_{0} \subseteq \mathcal{K}_{0}$ and $T \mathcal{H}_{1} \subseteq \mathcal{K}_{1}$.

2. If $\rho(\mathcal{A}) \mathcal{H}_{0}$ is dense in $\mathcal{H}$ and $T_{1}, T_{2}: \mathcal{H} \rightarrow \mathcal{K}$ are intertwining operators for $\rho$ and $\sigma$ such that $\left.T_{1}\right|_{\mathcal{H}_{0}}=\left.T_{2}\right|_{\mathcal{H}_{0}}$ then $T_{1}=T_{2}$. 
Proof. For $\eta=\eta_{0}+\eta_{1} \in \mathcal{K}$, with $\eta_{j} \in \mathcal{K}_{j}$, we put $Q_{j} \eta:=\eta_{j}$. We need to prove that both the operators $B:=\left.Q_{1} T\right|_{\mathcal{H}_{0}}$ and $C:=\left.Q_{0} T\right|_{\mathcal{H}_{1}}$ are zero. But $B$ (resp $C$ ) intertwines $\left.\rho_{0}\right|_{\mathcal{H}_{0}}$ and $\left.\sigma_{0}\right|_{\mathcal{H}_{1}}$ (resp. $\left.\rho_{0}\right|_{\mathcal{H}_{1}}$ and $\left.\rho_{0}\right|_{\mathcal{K}_{0}}$ ), which implies that $B=0$ and $C=0$ and proves the first assertion.

For the second one we note that for $a \in \mathcal{A}$ and $\xi \in \mathcal{H}_{0}$ we have

$$
T_{1} \rho(a) \xi=\sigma(a) T_{1} \xi=\sigma(a) T_{2} \xi=T_{2} \rho(a) \xi
$$

which implies that $T_{1}=T_{2}$.

Theorem 5.10. Suppose that $\pi_{0}$ and $\sigma_{0}$ are two representations of $\mathcal{A}(\tau)$ which are disjoint from the regular representation of $\mathcal{A}(\tau)$ and let $\pi$ and $\sigma$ be the induced representations of $G$.

1. If $\pi_{0}$ and $\sigma_{0}$ are not equivalent then so are $\pi$ and $\sigma$.

2. If $\pi_{0}$ and $\sigma_{0}$ are disjoint then so are $\pi$ and $\sigma$.

Proof. If $T: \mathcal{H} \rightarrow \mathcal{K}$ is an intertwining operator between $\pi$ and $\sigma$ then, by the previous lemma, $T\left(\mathcal{H}_{0}\right) \subseteq \mathcal{K}_{0}$, and hence $T \mid \mathcal{H}_{0}$ intertwines $\pi_{0}$ and $\sigma_{0}$. Therefore if $\pi_{0}$ and $\sigma_{0}$ are not equivalent then $\left.T\right|_{\mathcal{H}_{0}}$, and hence $T$ cannot be an isomorphism. Moreover, if $\pi_{0}$ and $\sigma_{0}$ are disjoint then $\left.T\right|_{\mathcal{H}_{0}}=0$ and for $\xi \in \mathcal{H}_{0}, x \in G$ we have $T \pi(x) \xi=\sigma(x) T \xi=0$, which implies that $T=0$.

Recall that a representation $\pi$ of a $*$-algebra $\mathcal{A}$ is said to be weakly contained in a representation $\sigma$ of $\mathcal{A}$ if $\|\pi(a)\| \leq\|\sigma(a)\|$ for every $a \in \mathcal{A}$. This is equivalent to saying that $\operatorname{ker}(\sigma) \subseteq \operatorname{ker}(\pi)$, where $\operatorname{ker}(\pi)$ and $\operatorname{ker}(\sigma)$ denote the kernel of the extension of $\pi$ and $\sigma$ to the enveloping $C^{*}$-algebra.

Proposition 5.11. Suppose that $\pi_{0}$ and $\sigma_{0}$ are $*$-representations of $\mathcal{A}(\tau)$ and let $\pi$ and $\sigma$ be the induced representations of $G=*_{i \in I} G_{i}$.

$i$. If $\pi_{0}$ is weakly contained in $\sigma_{0}$ then $\pi$ is weakly contained in $\sigma$.

ii. If $\pi$ is weakly contained in $\sigma$ then $\pi_{0}$ is weakly contained in $\sigma_{0} \oplus \lambda_{0}$.

In particular, $\pi$ is weakly contained in the regular representation of $G$ if and only if $\pi_{0}$ is weakly contained in the regular representation $\lambda_{0}$ of $\mathcal{A}(\tau)$.

Proof. Suppose that $\pi_{0}$ is weakly contained in $\sigma_{0}$ and decompose $\pi_{0}$ into a direct sum $\oplus_{\alpha \in A} \pi_{0}^{\alpha}$ of cyclic representations. For each $\alpha \in A$ we fix a unit cyclic vector $\xi^{\alpha}$ for $\pi_{0}^{\alpha}$ and let $\phi^{\alpha}$ be the corresponding state on $\mathcal{A}(\tau)$, i.e. $\phi^{\alpha}(a)=\left\langle\pi_{0}^{\alpha}(a) \xi^{\alpha}, \xi^{\alpha}\right\rangle$ for $a \in \mathcal{A}(\tau)$. Then $\pi=\oplus_{\alpha \in A} \pi^{\alpha}$, where $\pi^{\alpha}$ is induced from $\pi_{0}^{\alpha}$, and $\xi^{\alpha}$ is a cyclic vector also for $\pi^{\alpha}$. We need to show that every $\pi^{\alpha}$ is weakly contained in $\sigma$. Then the corresponding positive definite function on $G$ is $x \mapsto \phi^{\alpha}\left(\delta_{t(x)}\right)$. Since each $\pi_{0}^{\alpha}$ is weakly contained in $\sigma_{0}$ there is a sequence $\zeta_{n}^{\alpha}$ of vectors in the space of $\sigma_{0}$ such that $\lim _{n \rightarrow \infty}\left\langle\sigma_{0}(a) \zeta_{n}^{\alpha}, \zeta_{n}^{\alpha}\right\rangle=\phi^{\alpha}(a)$ for every $a \in \mathcal{A}(\tau)$. Then for every $x \in G$ we have

$$
\lim _{n \rightarrow \infty}\left\langle\sigma(x) \zeta_{n}^{\alpha}, \zeta_{n}^{\alpha}\right\rangle=\lim _{n \rightarrow \infty}\left\langle\sigma_{0}\left(\delta_{t(x)}\right) \zeta_{n}^{\alpha}, \zeta_{n}^{\alpha}\right\rangle=\phi^{\alpha}\left(\delta_{t(x)}\right)
$$

which means that $\pi_{\alpha}$ is weakly contained in $\sigma$.

If $\pi$ is weakly contained in $\sigma$ then $\operatorname{ker}(\sigma) \subseteq \operatorname{ker}(\pi)$ (the kernels are meant with respect to the full $C^{*}$-algebra of $G$ ) and hence $\operatorname{ker}\left(\left.\sigma\right|_{C^{*}(\mathcal{A}(\tau))}\right) \subseteq \operatorname{ker}\left(\left.\pi\right|_{C^{*}(\mathcal{A}(\tau))}\right)$. But the restriction of $\pi$ (resp. $\sigma$ ) to $C^{*}\left(\mathcal{A}(\tau)\right.$ ) is a direct sum of $\pi_{0}$ (resp. $\sigma_{0}$ ) and a subrepresentation of a multiple of $\lambda_{0}$ (Lemma 5.7). Therefore we have $\operatorname{ker}\left(\sigma_{0}\right) \cap \operatorname{ker}\left(\lambda_{0}\right) \subseteq$ $\operatorname{ker}\left(\pi_{0}\right) \cap \operatorname{ker}\left(\lambda_{0}\right) \subseteq \operatorname{ker}\left(\pi_{0}\right)$, which proves the second statement. 


\section{IRREDUCIBILITy OF THE INDUCED REPRESENTATION}

Throughout this section we fix a family $\left\{G_{i}\right\}_{i \in I}$ of groups and a representation $\pi_{0}$ of $\mathcal{A}(\tau)$, acting on a Hilbert space $\mathcal{H}_{0}$, where $\tau_{i}:=\left(\left|G_{i}\right|-1\right)^{-1}$, and therefore a family $\left\{P_{i}\right\}_{i \in I}$ of projections on $\mathcal{H}_{0}$, such that $\pi_{0}\left(\delta_{i}\right)=\left(1+\tau_{i}\right) P_{i}-\tau_{i}$ Id. We are going to study irreducibility of the induced representation $\pi$ of the group $G=*_{i \in I} G_{i}$.

Proposition 6.1. Assume that the representation $\pi_{0}$ (and hence the family of projections $\left\{P_{i}\right\}_{i \in I}$ ) is topologically irreducible (i.e. there is no nontrivial invariant closed subspace) and that there exists $i_{0} \in I$ such that $G_{i_{0}}$ is infinite and $P_{i_{0}} \neq 0$. Then $\pi$ is topologically irreducible.

Proof. Define operators $T_{i}$ on $\mathcal{H}$ by

$$
T_{i}= \begin{cases}\frac{1}{\left|G_{i}\right|} \sum_{g \in G_{i}} \pi(g) & \text { if } G_{i} \text { is finite } \\ \lim _{N \rightarrow \infty} \frac{1}{N} \sum_{n=1}^{N} \pi\left(g_{n, i}\right) & \text { otherwise }\end{cases}
$$

where, as before, $\left\{g_{n, i}\right\}$ is a sequence of distinct elements in $G_{i}$ and the convergence is in the strong sense (cf. Theorem 2.2 in [M2]). Then $T_{i}$ is a projection, $\left.T_{i}\right|_{\mathcal{H}_{0}}=P_{i}$ and $\left.T_{i}\right|_{\mathcal{H}_{0}^{\perp}}$ is an orthogonal projection. Moreover, if $G_{i}$ is infinite then $\left.T_{i}\right|_{\mathcal{H}_{0}^{\perp}}=0$.

Assume that $M$ is an invariant closed subspace of $\mathcal{H}$. Then $T_{i} M \subseteq M$ for every $i \in I$. If $T_{i_{0}} M \neq 0$ then the space $M_{0}:=M \cap \mathcal{H}_{0} \neq 0$ is invariant for all $T_{i}$, and hence for all $P_{i}$. This implies $M_{0}=\mathcal{H}_{0}$ and consequently $M=\mathcal{H}$.

Now assume that $T_{i_{0}} M=0$. This gives $T_{i_{0}} T_{i} M=0$, which means that $T_{i}^{*} T_{i_{0}}^{*} \mathcal{H}=$ $P_{i}^{*} P_{i_{0}}^{*} \mathcal{H}_{0}$ is orthogonal to $M$ for every $i \in I$. Since the family $\left\{P_{i}\right\}_{i \in I}$ (and hence $\left.\left\{P_{i}^{*}\right\}_{i \in I}\right)$ is irreducible in $\mathcal{H}_{0}$ and $P_{i_{0}} \neq 0$ we have $\mathcal{H}_{0} \perp M$, which implies $M=\{0\}$.

From now on we will be assuming that $\pi_{0}$ is a $*$-representation of $\mathcal{A}(\tau)$, hence all $P_{i}$ 's are selfadjoint and therefore the induced representation $\pi$ of $G$ is unitary.

Lemma 6.2. Let $\mathcal{A}_{1}$ be a *-subalgebra of a *-algebra $\mathcal{A}_{2}$ and let $\rho: \mathcal{A}_{2} \rightarrow \mathcal{B}(\mathcal{H})$ be a*-representation with a cyclic vector $\xi_{0}$. Assume that $\mathcal{H}_{1} \subseteq \mathcal{H}$ is an $\mathcal{A}_{1}$-invariant, closed subspace of $\mathcal{H}$, with $\xi_{0} \in \mathcal{H}_{1}$, such that the representation $\left.\rho\right|_{\mathcal{A}_{1}}$ is irreducible on $\mathcal{H}_{1}$ and occurs in $\mathcal{H}$ only once. Then $\rho$ is an irreducible representation of $\mathcal{A}_{2}$.

Proof. Let $M$ be an $\mathcal{A}_{2}$-invariant closed subspace of $\mathcal{H}$ and let $P$ be the orthogonal projection onto $M$. Then $P \rho(a)=\rho(a) P$ for every $a \in \mathcal{A}_{2}$. Indeed, for $\zeta, \eta \in \mathcal{H}$ we have

$$
\begin{aligned}
\langle P \rho(a) \zeta, \eta\rangle & =\langle\rho(a) \zeta, P \eta\rangle=\left\langle\zeta, \rho\left(a^{*}\right) P \eta\right\rangle \\
& =\left\langle P \zeta, \rho\left(a^{*}\right) P \eta\right\rangle=\langle\rho(a) P \zeta, P \eta\rangle=\langle\rho(a) P \zeta, \eta\rangle .
\end{aligned}
$$

If $P \mathcal{H}_{1}=\{0\}$ then, in particular, $P \xi_{0}=0$ and hence $M=\{0\}$. Assume that $M_{1}:=P \mathcal{H}_{1} \neq\{0\}$. Then one can observe that $\left.\rho\right|_{\mathcal{A}_{1}}$ acts irreducibly on $M_{1}$ (for if $M_{0}$ is an $\mathcal{A}_{1}$-invariant subspace of $M_{1}$ then so is $\left.P^{-1}\left(M_{0}\right) \cap \mathcal{H}_{1}\right)$ and that $P_{1}:=\left.P\right|_{\mathcal{H}_{1}}$ is an isomorphism $\mathcal{H}_{1} \rightarrow M_{1}$ (for $\operatorname{Ker} P_{1}$ is an $\mathcal{A}_{1}$-invariant subspace of $\mathcal{H}_{1}$ ). By our assumption this implies $\mathcal{H}_{1}=M_{1} \subseteq M$ and consequently $M=\mathcal{H}$ as $\xi_{0}$ lies in $\mathcal{H}_{1}$.

Denote $I^{\text {fin }}:=\left\{i \in I: G_{i}\right.$ is finite $\}, G^{\text {fin }}:=*_{i \in I^{\text {fin }}} G_{i}$ and let $\left(\mathcal{H}^{\text {fin }}=\bigoplus_{w \in G_{\text {fin }}} \mathcal{H}_{w}^{\text {fin }}, \pi^{\text {fin }}\right)$ be the induced representation of $G^{\text {fin }}$ related to the family $\left\{P_{i}\right\}_{i \in I^{\text {fin }}}$. There is a natural embedding of $\mathcal{H}^{\text {fin }}$ into $\mathcal{H}$. Namely, if we put $\mathcal{H}_{1}:=\oplus_{i \in I \backslash I^{\text {fin }}} \mathcal{H}_{i}$ then $\mathcal{H}_{w}=\mathcal{H}_{w}^{\text {fin }} \oplus \mathcal{H}_{1}$ for every $w \in G^{\text {fin }}$. The orthogonal complement of $\mathcal{H}^{\text {fin }}$ in $\mathcal{H}$ is $\ell^{2}\left(G^{\text {fin }}, \mathcal{H}_{1}\right) \oplus \bigoplus_{w \in G \backslash G^{\text {fin }}} \mathcal{H}_{w}$ 
and $\left.\pi\right|_{G^{\text {fin }}}$ acts on this subspace as a multiple of the regular representation, while $\left.\pi\right|_{G^{\text {fin }}}$ on $\mathcal{H}^{\text {fin }}$ is just $\pi^{\text {fin }}$.

Lemma 6.3. Assume that $\left|I^{\mathrm{fin}}\right| \geq 2, P_{i}=0$ for every $i \in I \backslash I^{\text {fin }}$ and that the representation $\pi^{\mathrm{fin}}$ of $G^{\mathrm{fin}}$ is irreducible. Then $\pi$ is irreducible too.

Proof. First of all we note that, by Theorem 5.5.ii, $\pi_{0}$ is irreducible. If $\left|I^{\text {fin }}\right| \geq 2$ then $G^{\text {fin }}$ is infinite so no irreducible representation of $G^{\text {fin }}$ can be contained in the orthogonal complement of $\mathcal{H}^{\text {fin }}$ in $\mathcal{H}$. Note also that every nonzero vector $\xi_{0} \in \mathcal{H}_{0}$ is cyclic for $\pi$. Indeed, by irreducibility of $\pi^{\text {fin }}$, the closure $M$ of $\operatorname{lin}\left\{\pi(x) \xi_{0}: x \in G\right\}$ contains $\mathcal{H}^{\text {fin }}$. In particular $\mathcal{H}_{0} \subseteq M$, which, by Theorem 5.5.vi, implies $M=\mathcal{H}$. Applying the previous lemma to $\mathcal{A}_{1}:=\mathcal{F}\left(G^{\text {fin }}\right)$ and $\mathcal{A}_{2}:=\mathcal{F}(G)$ we see that $\pi$ is irreducible.

For $i \in I \cup\{0\}$ we define a function $\phi_{i}$ on $S(I)$ by

$$
\phi_{0}\left(i_{1} i_{2} \ldots i_{m}\right):=\left(-\tau_{i_{1}}\right)\left(-\tau_{i_{2}}\right) \ldots\left(-\tau_{i_{m}}\right)
$$

and for $i \in I$

$$
\phi_{i}\left(i_{1} i_{2} \ldots i_{m}\right):=a_{i_{1}} a_{i_{2}} \ldots a_{i_{m}}
$$

where $a_{i}:=1$ and $a_{j}:=-\tau_{j}$ for $j \in I \backslash\{i\}$. We are now ready to present the main theorem of this paper:

Theorem 6.4. Suppose that we are given a free product group $G:=*_{i \in I} G_{i}$ and an irreducible *-representation $\pi_{0}: \mathcal{A}(\tau) \rightarrow \mathcal{B}\left(\mathcal{H}_{0}\right)$ of the algebra $\mathcal{A}(\tau)$, where $\tau_{i}:=\left(\left|G_{i}\right|-\right.$ $1)^{-1}$. Then the induced unitary representation $\pi$ of $G$ is irreducible unless $\pi_{0}$ is contained in the regular representation $\lambda_{0}$ of $\mathcal{A}(\tau)$, that is unless either

1) $\mathcal{H}_{0}=\mathbb{C}$ and $\pi_{0}\left(\delta_{i}\right)=-\tau_{i}$ for every $i \in I$ and

$$
\sum_{i \in I} \frac{1}{\left|G_{i}\right|}<1
$$

2) or $\mathcal{H}_{0}=\mathbb{C}$ and there is $i_{0} \in I$ such that $\pi_{0}\left(\delta_{i_{0}}\right)=1, \pi_{0}\left(\delta_{i}\right)=-\tau_{i}$ for all $i \in I \backslash\left\{i_{0}\right\}$ and

$$
\sum_{i \in I \backslash\left\{i_{0}\right\}} \frac{1}{\left|G_{i}\right|}<\frac{1}{\left|G_{i_{0}}\right|} .
$$

Proof. If $\pi_{0}$ is irreducible and contained in $\lambda_{0}$ then $\operatorname{dim} \pi_{0}=1$ (Theorem 2.11) and either $\pi_{0}\left(\delta_{i}\right)=-\tau_{i}$ for every $i \in I$ and $\sum_{i \in I} \frac{\tau_{i}}{1+\tau_{i}}<1$ or there is $i_{0} \in I$ such that $\pi_{0}\left(\delta_{i_{0}}\right)=1$, $\pi_{0}\left(\delta_{i}\right)=-\tau_{i}$ for $i \in I \backslash\{e\}$ and $\frac{1}{1+\tau_{i_{0}}}+\sum_{i \in I \backslash\left\{i_{0}\right\}} \frac{\tau_{i}}{1+\tau_{i}}<1$. The corresponding character ( $\phi_{0}$ in the first case and $\phi_{i_{0}}$ in the second) of $S(\tau)$ can be written as $\phi=f_{0} \cdot \tau$ and $f_{0} \in \ell^{2}(\tau)$, which means that the composition $\phi \circ t$ belongs to $\ell^{2}(G)$ and hence $\pi$ is not irreducible.

Now assume that $\pi_{0}$ is irreducible and not contained in $\lambda_{0}$. If there is $i \in I \backslash I^{\text {fin }}$ such that $\pi_{0}\left(\delta_{i}\right) \neq 0$ then $\pi$ is irreducible in view of Proposition 6.1. If, on the other hand, $\pi_{0}\left(\delta_{i}\right)=0$ for every $i \in I \backslash I^{\text {fin }}$ then our assumptions on $\pi_{0}$ imply that $\left|I^{\text {fin }}\right| \geq 2$. Now we observe that any nonzero vector $\xi \in \mathcal{H}_{0}$ is cyclic for $\pi^{\text {fin }}$. Indeed, by irreducibility of $\pi_{0}$ the closure of $\left\{\pi_{0}(a) \xi: a \in \mathcal{A}(\tau)\right\}=\left\{\pi_{0}(a) \xi: a \in \mathcal{A}\left(\left.\tau\right|_{I^{\text {fin }}}\right)\right\}$ is $\mathcal{H}_{0}$. Hence, by Theorem 5.5.vi, $\xi$ is cyclic for $\pi^{\text {fin }}$. Applying Lemma 6.2 to $\mathcal{A}_{1}:=\mathcal{F}_{t}\left(G^{\text {fin }}\right) \cong \mathcal{A}\left(\left.\tau\right|_{I^{\text {fin }}}\right)$ and $\mathcal{A}_{2}:=\mathcal{F}\left(G^{\mathrm{fin}}\right)$ we see, in view of Lemma 5.7 , that $\pi^{\mathrm{fin}}$ is irreducible. Now we conclude the proof by using Lemma 6.3 . 
Corollary 6.5. Let $\mathcal{P}^{1}(G)$ (resp. $\left.\mathcal{P}_{t}^{1}(G)\right)$ denote the convex set of all (type-dependent) positive definite functions $\phi$ on $G=*_{i \in I} G_{i}$ with $\phi(e)=1$ and let $\operatorname{ex}^{1}(G)$ (resp. $\left.\operatorname{ex} \mathcal{P}_{t}^{1}(G)\right)$ denote the set of its extreme points.

1. If

$$
\sum_{i \in I} \frac{1}{\left|G_{i}\right|} \geq 1
$$

then $\operatorname{ex}_{t}^{1}(G) \subseteq \operatorname{ex}^{1}(G)$.

2. If

$$
\sum_{i \in I} \frac{1}{\left|G_{i}\right|}<1 \quad \text { but } \quad \sum_{i \in I \backslash\{j\}} \frac{1}{\left|G_{i}\right|} \geq \frac{1}{\left|G_{j}\right|}
$$

for every $j \in I$ then $\operatorname{ex}_{t}^{1}(G) \backslash \operatorname{ex} \mathcal{P}^{1}(G)=\left\{\phi_{0} \circ t\right\}$.

3. Finally, if there is $i_{0} \in I$ such that

$$
\sum_{i \in I \backslash\left\{i_{0}\right\}} \frac{1}{\left|G_{i}\right|}<\frac{1}{\left|G_{i_{0}}\right|}
$$

then $\operatorname{ex}_{t}^{1}(G) \backslash \operatorname{ex}^{1}(G)=\left\{\phi_{0} \circ t, \phi_{i_{0}} \circ t\right\}$.

In another words, if a type-dependent function $\phi \circ t$ belongs to $\operatorname{ex}_{t}^{1}(G)$ then $\phi \circ t \in$ $\operatorname{ex}^{1}(G)$ holds if and only if $\phi \circ t \notin \ell^{2}(G)$.

The exceptional representations from points (1) and (2) of Theorem 6.4 have been studied in $[\mathrm{K}]$, and the positive definite function $\phi_{0} \circ t$, in the case when the groups $G_{i}$ are unipotent, appears in Lemma 1 in [T1]. The case when all $G_{i}$ 's are infinite was studied in [M2].

\subsection{Nonunitary representations.}

For nonunitary representations various definitions of irreducibility are not equivalent.

Definition 6.6. Let $\rho$ be a representation of a complex algebra $\mathcal{A}$ acting on a Hilbert space $\mathcal{K}$. Then $\rho$ is said to be

1. algebraically irreducible if there is no nontrivial invariant subspace of $\mathcal{K}$,

2. topologically irreducible if there is no nontrivial closed invariant subspace of $\mathcal{K}$,

3. fully irreducible if the closure of $\rho(\mathcal{A})$ in the strong operator topology coincides with $\mathcal{B}(\mathcal{K})$.

In view of the Burnside's theorem these notions coincide if $\operatorname{dim} \mathcal{K}$ is finite. In general, full irreducibility implies topological irreducibility.

Lemma 6.7. Let $\rho_{0}: \mathcal{A} \rightarrow \mathcal{B}\left(\mathcal{K}_{0}\right)$ be a finite dimensional irreducible representation of $a *$-algebra $\mathcal{A}$ which is not equivalent to a $*$-representation and let $\rho_{1}: \mathcal{A} \rightarrow \mathcal{B}\left(\mathcal{K}_{1}\right)$ be a $*$-representation of $\mathcal{A}$. Then there is a sequence $a_{n} \in \mathcal{A}$ satisfying $\left\|\rho_{0}\left(a_{n}\right)-\operatorname{Id}_{\mathcal{K}_{0}}\right\| \rightarrow 0$ and $\left\|\rho_{1}\left(a_{n}\right)\right\| \rightarrow 0$.

Proof. Assume that there is a constant $L$ such that

$$
\left\|\rho_{0}(a)\right\| \leq L\left\|\rho_{1}(a)\right\| \quad \text { for every } a \in \mathcal{A} .
$$

Then we take $\mathcal{B}$ to be the norm closure of $\rho_{1}(\mathcal{A})$. It is a $C^{*}$-algebra and by $(*)$ we can extend $\rho_{0}$ to a representation of $\mathcal{B}$. However every algebraically irreducible representation of a $C^{*}$-algebra is equivalent to a $*$-representation (see Corollary 2.9.6 in [Di]). Therefore $(*)$ can not be true. Hence there is a sequence $c_{n} \in \mathcal{A}$ satisfying $\left\|\rho_{0}\left(c_{n}\right)\right\|=1$ and $\left\|\rho_{1}\left(c_{n}\right)\right\| \rightarrow 0$. As $\operatorname{dim} \mathcal{K}_{0}$ is finite we may in addition assume that $\left\|\rho_{0}\left(c_{n}\right)-C\right\| \rightarrow 0$ 
for some $C \in \mathcal{B}\left(\mathcal{K}_{0}\right)$ with $\|C\|=1$. Since $\rho_{2}$ is fully irreducible, there exist $b_{k}, d_{k} \in \mathcal{A}$, $k \leq M$, such that

$$
\sum_{k=1}^{M} \rho_{0}\left(b_{k}\right) C \rho_{0}\left(d_{k}\right)=\operatorname{Id}_{\mathcal{K}_{0}} .
$$

Now we can conclude the proof by putting $a_{n}:=\sum_{k=1}^{M} b_{k} c_{n} d_{k}$.

Now we are ready to prove

Theorem 6.8. Assume that we are given an irreducible representation $\left(\pi_{0}, \mathcal{H}_{0}\right)$ of $\mathcal{A}(\tau)$, with $\operatorname{dim} \mathcal{H}_{0}<\infty$, which is not equivalent to a*-representation. Then the induced representation $\pi$ of the free product group $G=*_{i \in I} G_{i}$ is fully irreducible.

First we prove a weaker statement.

Lemma 6.9. Under the assumptions of Theorem 6.8, $\pi$ is topologically irreducible.

Proof. Consider the representation $\left(\widetilde{\pi}_{0}, \mathcal{H}\right)$ of $\mathcal{A}(\tau)$ defined by formula (5.3) and its restrictions $\rho_{0}$ and $\rho_{1}$ to $\mathcal{H}_{0}$ and $\mathcal{H}_{0}^{\perp}$ respectively, so that $\rho_{0}=\pi_{0}$ and $\rho_{1}$ is a multiple of $\lambda_{0}$ (Lemma 5.7). In view of Lemma 6.7 the orthogonal projection $P_{0}$ of $\mathcal{H}$ to $\mathcal{H}_{0}$ belongs to the norm closure of $\widetilde{\pi}_{0}(\mathcal{A}(\tau))$.

Let $M$ be a $G$-invariant subspace of $\mathcal{H}$ and denote $M_{0}:=P_{0} M \subseteq M$.

If $M_{0} \neq\{0\}$ then $M_{0}$ it is a nontrivial closed subspace of $\mathcal{H}_{0}$ which is $\mathcal{A}(\tau)$-invariant. Hence $M_{0}=\mathcal{H}_{0}$, which means that $H_{0} \subseteq M$. For fixed $i \in I$ we have $\pi(g) \mathcal{H}_{0} \subseteq M$ for each $g \in G_{i}$, which leads $\delta_{e} \otimes \mathcal{H}_{i} \subseteq M$. Consequently, $\delta_{e} \otimes \mathcal{H}_{e} \subseteq M$ and for any $w \in G$ we have $\delta_{w} \otimes \mathcal{H}_{w}=\pi(w)\left(\delta_{e} \otimes \mathcal{H}_{w}\right)$. Therefore $M=\mathcal{H}$.

Now assume that $P_{0} M=\{0\}$, i.e. $M \perp \mathcal{H}_{0}$. For fixed $i \in I$ and for every $g \in G_{i}$ we have $\pi(g) M \subseteq M \perp \mathcal{H}_{0}$, so that $M \perp \delta_{e} \otimes \mathcal{H}_{i}$, and hence $M \perp \delta_{e} \otimes \mathcal{H}_{e}$. Now for $w \in G$ we have $\pi\left(w^{-1}\right) M \subseteq M \perp \delta_{e} \otimes \mathcal{H}_{e}$, which yields $M \perp \delta_{w} \otimes \mathcal{H}_{w}$ and we conclude that $M=\{0\}$.

Proof of theorem 6.8. Let $\mathcal{B}$ denote the closure of $\operatorname{lin}\{\pi(x): x \in G\}$ in the strong operator topology of $\mathcal{B}(\mathcal{H})$. Then $P_{0} \in \mathcal{B}$ by the first lemma and, consequently, $B P_{0} \in \mathcal{B}$ for every $B \in \mathcal{B}\left(\mathcal{H}_{0}\right)$. In particular, $\zeta_{0} \otimes \eta_{0} \in \mathcal{B}$ for fixed $\zeta_{0}, \eta_{0} \in \mathcal{H}_{0} \backslash\{0\}$. Now take any $\zeta, \eta \in \mathcal{H}$. Due to the topological irreducibility of the family $\mathcal{B}$ (and hence of $\left.\mathcal{B}^{*}:=\left\{B^{*}: B \in \mathcal{B}\right\}\right)$ there are $S_{n}, T_{n} \in \mathcal{B}$ such that $S_{n} \zeta_{0} \rightarrow \zeta$ and $T_{n}^{*} \eta_{0} \rightarrow \eta$, which implies

$$
S_{n}\left(\zeta_{0} \otimes \eta_{0}\right) T_{n}=\left(S_{n} \zeta_{0}\right) \otimes\left(T_{n}^{*} \eta_{0}\right) \rightarrow \zeta \otimes \eta
$$

in the strong topology. Therefore $\mathcal{B}$ contains all operators of finite rank, so $\mathcal{B}=\mathcal{B}(\mathcal{H})$.

\section{FREE PRODUCT OF TWO GROUPS}

This section is devoted to the case when $|I|=2$, say $I=\{+,-\}$. Here we will write $S(+,-)$ and $\mathcal{A}\left(\tau_{+}, \tau_{-}\right)$instead of $S(I)$ and $\mathcal{A}(\tau)$. A word of the form $u=+-\cdots \pm$ (resp. $u=-+\cdots \pm$ ), with $|u|=m$, will be denoted $+m$ (resp. $-m$ ) and here we will denote by 0 the empty word.

Cartwright and Soardi [CS] (see also [ML, T2]) introduced a family of spherical functions $\phi_{\lambda}\left(\lambda\right.$ is a complex parameter) on the free product of two groups: $\mathbb{Z}_{r} * \mathbb{Z}_{s}$, where $r>s \geq 2$. It was shown in [M1] that such a function is positive definite if and only if 
$\lambda \in[-2, s-2] \cup[r-2, r+s-2]$. Here our aim is to study in detail the corresponding family of representations.

First we are interested in finding all irreducible representations of $\mathcal{A}\left(\tau_{+}, \tau_{-}\right)$, therefore in finding all (equivalence classes of) irreducible pairs $\left(P_{+}, P_{-}\right)$of projections on a Hilbert space $\mathcal{H}_{0}$. Two such pairs: $\left(P_{+}, P_{-}\right)$on $\mathcal{H}_{0}$ and $\left(Q_{+}, Q_{-}\right)$on $\mathcal{K}_{0}$ are said to be equivalent if there is an invertible operator $T: \mathcal{H}_{0} \rightarrow \mathcal{K}_{0}$ such that $T P_{+}=Q_{+} T$ and $T P_{-}=Q_{-} T$. Putting $Z:=2 P_{+}-\mathrm{Id}$ and $T:=\left(2 P_{+}-\mathrm{Id}\right)\left(2 P_{-}-\mathrm{Id}\right)$ we have $Z^{2}=\mathrm{Id}$ and $Z T Z=T^{-1}$ so our question is equivalent to finding all (equivalence classes of) irreducible representations of the semidirect product $\mathbb{Z}_{2} \ltimes \mathbb{Z}$. Unitary irreducible representations of $\mathbb{Z}_{2} \ltimes \mathbb{Z}$ are known to be at most two-dimensional. We note that the same holds without unitarity if we assume that $\operatorname{dim} \mathcal{H}_{0}$ is finite, as we now see.

Lemma 7.1. Let $\left(P_{+}, P_{-}\right)$be an irreducible pair of projections in a finitely dimensional Hilbert space $\mathcal{H}_{0}$. Then $\operatorname{dim} \mathcal{H}_{0} \leq 2$. Moreover, two irreducible pairs $\left(P_{+}, P_{-}\right)$and $\left(Q_{+}, Q_{-}\right)$of projections on a two-dimensional Hilbert space $\mathcal{H}_{0}$ are equivalent if and only if $\operatorname{tr}\left(P_{+} P_{-}\right)=\operatorname{tr}\left(Q_{+} Q_{-}\right)$.

Proof. Put $Z:=2 P_{+}-\mathrm{Id}$ and $T:=\left(2 P_{+}-\mathrm{Id}\right)\left(2 P_{-}-\mathrm{Id}\right)$. Since $\operatorname{dim} \mathcal{H}_{0}$ is finite, $T$ has an eigenvector $\eta_{1} \neq 0$ so that $T \eta_{1}=\lambda \eta_{1}$, with $\lambda \neq 0$. Put $\eta_{2}:=Z \eta_{1}$. Then $Z \eta_{2}=\eta_{1}$ and

$$
T \eta_{2}=T Z \eta_{1}=Z T^{-1} \eta_{1}=\lambda^{-1} Z \eta_{1}=\lambda^{-1} \eta_{2}
$$

which implies that the vectors $\eta_{1}, \eta_{2}$ span an invariant subspace, hence $\operatorname{dim} \mathcal{H}_{0} \leq 2$. If $\lambda= \pm 1$ then $T\left(\eta_{1}+\eta_{2}\right)= \pm\left(\eta_{1}+\eta_{2}\right)$ and $Z\left(\eta_{1}+\eta_{2}\right)=\eta_{1}+\eta_{2}$ so $\operatorname{dim} \mathcal{H}_{0}=1$.

Now assume that $\lambda \neq \pm 1$. Then the vectors $\eta_{1}, \eta_{2}$ are linearly independent and $\operatorname{tr} T=\lambda+\lambda^{-1}$. If operators $W, S$ on $\mathcal{H}_{0}$ satisfy $W^{2}=\mathrm{Id}, W S W=S^{-1}$ and $\operatorname{tr} S=\lambda+\lambda^{-1}$ then, as before, $S$ has eigenvalues $\gamma$ and $\gamma^{-1}$ which satisfy $\gamma+\gamma^{-1}=\lambda+\lambda^{-1}$. This implies $\gamma=\lambda$ or $\gamma=\lambda^{-1}$, so there are $\xi_{1}, \xi_{2} \neq 0$ such that $S \xi_{1}=\lambda \xi_{1}, S \xi_{2}=\lambda^{-1} \xi_{2}$. Thus the map given by $\xi_{1} \mapsto \eta_{1}, \xi_{2} \mapsto \eta_{2}$ defines an equivalence between pairs $Z, T$ and $W, S$.

Therefore the family of all irreducible pairs $P_{+}, P_{-}$of projections on two-dimensional Hilbert space is parametrised by $w:=\operatorname{tr}\left(P_{+} P_{-}\right), w \in \mathbb{C} \backslash\{0,1\}$. Note that for unit vectors $\eta_{ \pm} \in \mathcal{H}_{0}$ and for $P_{ \pm}:=\eta_{ \pm} \otimes \eta_{ \pm}$we have $\operatorname{tr}\left(P_{+} P_{-}\right)=\left|\left\langle\eta_{+}, \eta_{-}\right\rangle\right|^{2}$ so the points from the interval $(0,1)$ correspond to pairs of orthogonal projections. We also include the parameters $w=0$ and $w=1$ when $\eta_{+} \perp \eta_{-}$or $\eta_{+}=\eta_{-}$respectively.

Now fix $\tau_{+}, \tau_{-} \geq 0$ and set $B_{ \pm}:=\left(1+\tau_{ \pm}\right) P_{ \pm}-\tau_{ \pm}$Id for a pair $\left(P_{+}, P_{-}\right)$with $\operatorname{tr}\left(P_{+} P_{-}\right)=w$. We denote by $\pi_{w}^{0}$ the representation of $\mathcal{A}\left(\tau_{+}, \tau_{-}\right)$for which $\pi_{w}^{0}\left(\delta_{ \pm}\right)=B_{ \pm}$. For $w \in\{0,1\}$ these representations can be decomposed into one-dimensional ones: $\pi_{0}^{0}=\pi_{01}^{0} \oplus \pi_{10}^{0}$ and $\pi_{1}^{0}=\pi_{00}^{0} \oplus \pi_{11}^{0}$, where $\pi_{\epsilon_{+} \epsilon_{-}}^{0}\left(\delta_{ \pm}\right)=\left(1+\tau_{ \pm}\right) \epsilon_{ \pm}-\tau_{ \pm}$. Therefore the family $\left\{\pi_{w}^{0}\right\}$, with $w \in(\mathbb{C} \backslash\{0,1\}) \cup\{0,1\}^{2}$, exhausts all finite-dimensional irreducible representations of $\mathcal{A}\left(\tau_{+}, \tau_{-}\right)$. The coefficient of $\pi_{\epsilon_{+} \epsilon_{-}}^{0}$ is the character:

$$
\widetilde{\phi}_{\epsilon_{+} \epsilon_{-}}\left(i_{1} i_{2} \ldots i_{m}\right)=a_{i_{1}} a_{i_{2}} \ldots a_{i_{m}}
$$

where $a_{i}=-\tau_{i}$ if $\epsilon_{i}=0$ and $a_{i}=1$ if $\epsilon_{i}=1$. Applying results from Section 2, concerning the left regular representation $\lambda_{0}$ of $\mathcal{A}(\tau)$, and Theorem 3.1, we have

\section{Proposition 7.2.}

1. $\pi_{00}^{0}$ is contained in $\lambda_{0}$ iff $\tau_{+} \tau_{-}<1$,

2. $\pi_{01}^{0}$ is contained in $\lambda_{0}$ iff $\tau_{+}<\tau_{-}$, 
3. $\pi_{10}^{0}$ is contained in $\lambda_{0}$ iff $\tau_{+}>\tau_{-}$,

4. $\pi_{11}^{0}$ is contained in $\lambda_{0}$ iff $\tau_{+} \tau_{-}>1$.

Equivalently, $\pi_{\epsilon_{+} \epsilon_{-}}^{0}$ is contained in $\lambda_{0}$ iff $\left(\epsilon_{+}+\epsilon_{-}-1\right)\left(1-\tau_{+} \tau_{-}\right)<\left(\epsilon_{+}-\epsilon_{-}\right)\left(\tau_{+}-\tau_{-}\right)$. Moreover, $\pi_{\epsilon_{+} \epsilon_{-}}^{0}$ is weakly contained in $\lambda_{0}$ iff the same conditions hold, with " $<$ " replaced by " $\leq$ ".

For two-dimensional representations we need to study the eigenvalues of $B_{+} B_{-}$. We will base on the following elementary fact.

Lemma 7.3. Assume that $z=u+v \mathrm{i} \in \mathbf{C}$ and $a \geq 0$. Then both the inequalities $\left|z \pm \sqrt{z^{2}-a}\right| \leq 1$ hold if and only if either $a=1, v=0$ and $-1 \leq u \leq 1$ or $0 \leq a<1$ and $4 u^{2}(1+a)^{-2}+4 v^{2}(1-a)^{-2} \leq 1$. Strict inequalities $\left|z \pm \sqrt{z^{2}-a}\right|<1$ hold if and only if $0 \leq a<1$ and $4 u^{2}(1+a)^{-2}+4 v^{2}(1-a)^{-2}<1$.

The equality $\left|z+\sqrt{z^{2}-a}\right|=\left|z-\sqrt{z^{2}-a}\right|$ holds if and only if $z$ is real and $z^{2} \leq a$.

Proposition 7.4. The representation $\pi_{w}^{0}$ is uniformly bounded (i.e. the norms $\left\|\pi_{w}^{0}\left(\delta_{u}\right)\right\|$, $u \in S(I)$, are uniformly bounded) if and only if either $\tau_{+} \tau_{-}=1$ and $\left(\frac{1-\tau_{+}}{1+\tau_{+}}\right)^{2} \leq w \leq 1$ or $\tau_{+} \tau_{-}<1$ and $w=x+y$ i satisfies

$$
\frac{\left(\left(1+\tau_{+}\right)\left(1+\tau_{-}\right) x-\tau_{+}-\tau_{-}\right)^{2}}{\left(1+\tau_{+} \tau_{-}\right)^{2}}+\frac{\left(1+\tau_{+}\right)^{2}\left(1+\tau_{-}\right)^{2} y^{2}}{\left(1-\tau_{+} \tau_{-}\right)^{2}} \leq 1 .
$$

For $0<w<1$ the $*$-representation $\pi_{w}^{0}$ is weakly contained in $\lambda_{0}$ if and only if

$$
w \in\left[\frac{\left(\sqrt{\tau_{+}}-\sqrt{\tau_{-}}\right)^{2}}{\left(1+\tau_{+}\right)\left(1+\tau_{-}\right)}, \frac{\left(\sqrt{\tau_{+}}+\sqrt{\tau_{-}}\right)^{2}}{\left(1+\tau_{+}\right)\left(1+\tau_{-}\right)}\right] .
$$

Proof. We have $\operatorname{det}\left(B_{ \pm}\right)=-\tau_{ \pm}$so that $\operatorname{det}\left(B_{+} B_{-}\right)=\tau_{+} \tau_{-}$. We also have

$$
\operatorname{tr}\left(B_{+} B_{-}\right)=\left(1+\tau_{+}\right)\left(1+\tau_{-}\right) w-\left(\tau_{+}+\tau_{-}\right) .
$$

It remains to apply Lemma 7.3 to the eigenvalues

$$
\eta_{ \pm}=\frac{\operatorname{tr}\left(B_{+} B_{-}\right)}{2} \pm \sqrt{\left(\frac{\operatorname{tr}\left(B_{+} B_{-}\right)}{2}\right)^{2}-\operatorname{det}\left(B_{+} B_{-}\right)}
$$

of $B_{+} B_{-}$and to use Theorem 3.1.

Let us denote by $E\left(\tau_{+}, \tau_{-}\right)$the closed subset of the complex plane described by (7.1). Its boundary is an ellipse with the following basic points $(x, 0)$ :

$$
\begin{aligned}
\text { centre: } & x=\frac{\tau_{+}+\tau_{-}}{\left(1+\tau_{+}\right)\left(1+\tau_{-}\right)}, \\
\text {vertices: } & x=-\frac{\left(1-\tau_{+}\right)\left(1-\tau_{-}\right)}{\left(1+\tau_{+}\right)\left(1+\tau_{-}\right)} \quad \text { and } \quad x=1, \\
\text { foci: } & x=\frac{\left(\sqrt{\left.\tau_{+} \pm \sqrt{\tau_{-}}\right)^{2}}\right.}{\left(1+\tau_{+}\right)\left(1+\tau_{-}\right)} .
\end{aligned}
$$

Let $G$ be the free product of two groups, $G=G_{+} * G_{-}$, and put $\tau_{ \pm}:=\left(\left|G_{ \pm}\right|-1\right)^{-1}$. For the representation $\pi_{w}^{0}$ of $\mathcal{A}\left(\tau_{+}, \tau_{-}\right)$, with $w \in \mathbb{C} \backslash\{0,1\}$ or $w \in\{0,1\}^{2}$, let $\pi_{w}$ denote the induced representation of $G$. Then applying Theorem 5.5, 5.10, 6.4 and 6.8 we get immediately 
Theorem 7.5. 1. Assume that $w \in \mathbb{C} \backslash\{0,1\}$. Then

i. $\pi_{w}$ is fully irreducible.

ii. If $w_{1} \neq w_{2}$ then $\pi_{w_{1}}$ and $\pi_{w_{2}}$ are inequivalent.

iii. If $w \in \operatorname{Int} E\left(\tau_{+}, \tau_{-}\right)$then $\pi_{w}$ is uniformly bounded.

iv. $\pi_{w}$ is unitary if and only if $w \in(0,1)$.

2. For $w \in\{0,1\}^{2}$ the representation $\pi_{w}$ is unitary and

i. $\pi_{11}$ is irreducible.

ii. $\pi_{01}$ is irreducible if and only if $\left|G_{+}\right| \leq\left|G_{-}\right|$.

iii. $\pi_{10}$ is irreducible if and only if $\left|G_{+}\right| \geq\left|G_{-}\right|$.

iv. $\pi_{00}$ is irreducible if and only if $\left|G_{+}\right|=\left|G_{-}\right|=2$.

From now on we fix $\tau_{+}, \tau_{-} \geq 0$ and $\beta_{+}, \beta_{-}>0$. Assume that $\beta_{-}\left(1+\tau_{-}\right) \leq \beta_{+}\left(1+\tau_{+}\right)$. Set $\mu:=\beta_{+} \delta_{+}+\beta_{-} \delta_{-}$and denote by $\mathcal{A}(\tau, \mu)$ the commutative unital $*$-subalgebra of $\mathcal{A}(\tau)$ generated by $\mu$. Let $C^{*}(\tau, \mu)$ and $C_{r}^{*}(\tau, \mu)$ be the closure of $\mathcal{A}(\tau, \mu)$ in $C^{*}(\mathcal{A}(\tau))$ and $C_{r}^{*}(\mathcal{A}(\tau))$ (see remarks preceding Proposition 5.8) respectively.

Proposition 7.6. Denote by $\mathrm{sp}(\mu)$ and $\operatorname{sp}_{r}(\mu)$ the spectrum of $\mu$ in $C^{*}(\tau, \mu)$ and $C_{r}^{*}(\tau, \mu)$ or, equivalently, in $C^{*}(\mathcal{A}(\tau))$ and $C_{r}^{*}(\mathcal{A}(\tau))$, respectively. Then

$$
\begin{aligned}
\operatorname{sp}(\mu) & =\left[-\beta_{+} \tau_{+}-\beta_{-} \tau_{-},-\beta_{+} \tau_{+}+\beta_{-}\right] \cup\left[-\beta_{-} \tau_{-}+\beta_{+}, \beta_{+}+\beta_{-}\right], \\
\operatorname{sp}_{r}(\mu) & =\left[x_{0}-x_{+}, x_{0}-x_{-}\right] \cup\left[x_{0}+x_{-}, x_{0}+x_{+}\right] \cup \Upsilon,
\end{aligned}
$$

where $x_{0}:=\left(\beta_{+}\left(1-\tau_{+}\right)+\beta_{-}\left(1-\tau_{-}\right)\right) / 2$,

$$
x_{ \pm}:=\sqrt{\left(\frac{\beta_{+}\left(1+\tau_{+}\right)-\beta_{-}\left(1+\tau_{-}\right)}{2}\right)^{2}+\beta_{+} \beta_{-}\left(\sqrt{\tau_{+}} \pm \sqrt{\tau_{-}}\right)^{2}}
$$

and $\Upsilon$ consists of those points (at most two)

$$
\beta_{+}\left(1+\tau_{+}\right) \epsilon_{+}+\beta_{-}\left(1+\tau_{-}\right) \epsilon_{-}-\left(\beta_{+} \tau_{+}+\beta_{-} \tau_{-}\right),
$$

with $\epsilon_{ \pm} \in\{0,1\}$, for which $\left(\epsilon_{+}+\epsilon_{-}-1\right)\left(1-\tau_{+} \tau_{-}\right) \leq\left(\epsilon_{+}-\epsilon_{-}\right)\left(\tau_{+}-\tau_{-}\right)$.

Proof. Recall that the spectrum of an element $a_{0}$ in a commutative Banach algebra $\mathcal{A}$ is the set of all values $\phi\left(a_{0}\right)$, where $\phi$ runs over all multiplicative functionals of $\mathcal{A}$. If $\phi$ is a multiplicative functional on $C^{*}(\tau, \mu)$ then, regarded as a 1-dimensional representation, it can be extended to an irreducible $*$-representation of $C^{*}(\mathcal{A}(\tau))$ (see 2.10.2 in [Di]) and hence of $\mathcal{A}(\tau)$. Therefore we can say that there is $w \in[0,1]$ and a unit eigenvector $\xi$ for $\pi_{w}^{0}(\mu)$ such that $\phi(a)=\left\langle\pi_{w}(a) \xi, \xi\right\rangle$ for $a \in \mathcal{A}(\tau, \mu)$. Put $\alpha_{ \pm}:=\left(1+\tau_{ \pm}\right) \beta_{ \pm}$and $B:=\alpha_{+} P_{+}+\alpha_{-} P_{-}$. We have $P_{ \pm}=\eta_{ \pm} \otimes \eta_{ \pm}, w=\left|\left\langle\eta_{+}, \eta_{-}\right\rangle\right|^{2}$ and

$$
\begin{aligned}
& B \eta_{+}=\alpha_{+} \eta_{+}+\alpha_{-}\left\langle\eta_{+}, \eta_{-}\right\rangle \eta_{-}, \\
& B \eta_{-}=\alpha_{+}\left\langle\eta_{-}, \eta_{+}\right\rangle \eta_{+}+\alpha_{-} \eta_{-},
\end{aligned}
$$

so that the eigenvalues of $B$ are

$$
t_{ \pm}(w)=\frac{\alpha_{+}+\alpha_{-} \pm \sqrt{\left(\alpha_{+}-\alpha_{-}\right)^{2}+4 w \alpha_{+} \alpha_{-}}}{2} .
$$

If $w$ runs over $[0,1]$ then $t_{ \pm}(w)$ runs over $\left[0, \alpha_{-}\right] \cup\left[\alpha_{+}, \alpha_{+}+\alpha_{-}\right]$. Knowing that

$$
\pi_{w}^{0}(\mu)=B-\left(\tau_{+} \beta_{+}+\tau_{-} \beta_{-}\right) \mathrm{Id}
$$

we obtain the first statement.

For $\operatorname{sp}_{r}(\mu)$ we have to take into account those $\pi_{w}^{0}$ which are contained or weakly contained in the regular representation of $\mathcal{A}(\tau)$. 
Now we will study some coefficients of the representations $\pi_{w}^{0}$ and $\pi_{w}$. For a complex function $\phi$ on $S(I)$ and for $f \in \mathcal{F}(S(I))$ we define their dual right $\tau$-convolution $\phi \diamond_{\tau} f$ putting $\left(\phi \diamond_{\tau} f\right)(u)=\left\langle\phi, \delta_{u} *_{\tau} f^{\vee}\right\rangle$, where $f^{\vee}(u):=f\left(u^{*}\right)$.

Definition 7.7. Let $\tau_{+}, \tau_{-} \geq 0, \beta_{+}, \beta_{-}>0, \lambda \in \mathbb{C}$. A complex function $\phi$ on $S(+,-)$ is said to be $\left(\tau_{+}, \tau_{-}, \beta_{+}, \beta_{-} ; \lambda\right)$-spherical if

1) $\phi(e)=1$

2) $\phi\left(u^{*}\right)=\phi(u)$ for $u \in S(+,-)$;

3) $\phi \diamond_{\tau} \mu=\lambda \phi$, where $\tau=\left(\tau_{+}, \tau_{-}\right), \mu=\beta_{+} \delta_{+}+\beta_{-} \delta_{-}$.

It was shown in [M1], Proposition 4.2, that for $\lambda \neq x_{0}:=\left(\beta_{+}\left(1-\tau_{+}\right)+\beta_{-}\left(1-\tau_{-}\right)\right) / 2$ such a function $\phi$ does exist and is unique. If $\beta_{+}\left(1+\tau_{+}\right)=\beta_{-}\left(1+\tau_{-}\right)$and $\lambda=x_{0}$ then such a function exists but is not unique.

Fix $\tau_{+}, \tau_{-} \geq 0, \beta_{+}, \beta_{-}>0$ and let $\phi_{\lambda}$ denote the $\left(\tau_{+}, \tau_{-}, \beta_{+}, \beta_{-} ; \lambda\right)$-spherical function. If $\beta_{+}\left(1+\tau_{+}\right)=\beta_{-}\left(1+\tau_{-}\right)$then we define $\phi_{x_{0}}$ as the pointwise limit of $\phi_{\lambda}$ when $\lambda \rightarrow x_{0}$. Then, as it was explained in the proof of [M1], Theorem 4.5, the function $\phi=T_{\sigma \tau} \phi_{\lambda}$ (the map $T_{\sigma \tau}$ was defined in Section 1), for $\sigma_{ \pm}=0$, is the $\left(0,0, \alpha_{+}, \alpha_{-} ; \gamma\right)$-spherical function $\psi_{\gamma}$, where

$$
\alpha_{+}=\left(1+\tau_{+}\right) \beta_{+}, \quad \alpha_{-}=\left(1+\tau_{-}\right) \beta_{-}, \quad \gamma=\lambda+\tau_{+} \beta_{+}+\tau_{-} \beta_{-} .
$$

Now we are going to obtain spherical functions as coefficients of representations of $\mathcal{A}\left(\tau_{+}, \tau_{-}\right)$. Take $\mathcal{H}_{0}:=\mathbb{C}^{2}$, with an orthonormal basis $\zeta_{0}, \zeta_{1}$. For complex numbers $\theta, \omega$ define vectors

$$
\begin{gathered}
\zeta_{+}(\theta):=\cos \theta \cdot \zeta_{0}+\sin \theta \cdot \zeta_{1}, \quad \zeta_{-}(\theta):=\cos \theta \cdot \zeta_{0}-\sin \theta \cdot \zeta_{1}, \\
\xi(\omega):=\cos \omega \cdot \zeta_{0}+\sin \omega \cdot \zeta_{1} .
\end{gathered}
$$

If $\theta, \omega$ are real then the angle between $\zeta_{+}(\theta)$ and $\zeta_{-}(\theta)$ is $2 \theta$ and between $\zeta_{ \pm}(\theta)$ and $\xi(\omega)$ is $\theta \pm \omega$.

Now we define one-dimensional projections

$$
P_{+}:=\zeta_{+}(\theta) \otimes \zeta_{+}(\bar{\theta}), \quad P_{-}:=\zeta_{-}(\theta) \otimes \zeta_{-}(\bar{\theta}) .
$$

Then $\left[\zeta_{+}(\theta), \zeta_{-}(\bar{\theta})\right]=\left[\zeta_{-}(\theta), \zeta_{+}(\bar{\theta})\right]=\cos (2 \theta)$ and for any $u=i_{1} i_{2} \ldots i_{n} \in S(I) \backslash\{e\}$

$$
\left[P_{i_{1}} P_{i_{2}} \ldots P_{i_{n}} \xi(\omega), \xi(\bar{\omega})\right]=\cos z_{i_{1}} \cos ^{n-1}(2 \theta) \cos z_{i_{n}}
$$

where $z_{ \pm}=\theta \pm \omega$.

Now we choose the numbers $\theta, \omega$ in a special way. Assume that

$$
\cos ^{2}(\theta+\omega)=\frac{\gamma\left(\alpha_{-}-\gamma\right)}{\alpha_{+}\left(\alpha_{+}+\alpha_{-}-2 \gamma\right)}, \quad \cos ^{2}(\theta-\omega)=\frac{\gamma\left(\alpha_{+}-\gamma\right)}{\alpha_{-}\left(\alpha_{+}+\alpha_{-}-2 \gamma\right)} .
$$

Then we have

$$
\begin{gathered}
\alpha_{+} \cos ^{2}(\theta+\omega)+\alpha_{-} \cos ^{2}(\theta-\omega)=\gamma \\
\sin ^{2}(\theta+\omega)=\frac{\left(\alpha_{+}-\gamma\right)\left(\alpha_{+}+\alpha_{-}-\gamma\right)}{\alpha_{+}\left(\alpha_{+}+\alpha_{-}-2 \gamma\right)}, \quad \sin ^{2}(\theta-\omega)=\frac{\left(\alpha_{-}-\gamma\right)\left(\alpha_{+}+\alpha_{-}-\gamma\right)}{\alpha_{-}\left(\alpha_{+}+\alpha_{-}-2 \gamma\right)}
\end{gathered}
$$

and

$$
\alpha_{+}^{2} \sin ^{2}(\theta+\omega) \cos ^{2}(\theta+\omega)=\alpha_{-}^{2} \sin ^{2}(\theta-\omega) \cos ^{2}(\theta-\omega) .
$$

We assume in addition that

$$
\alpha_{+} \sin (\theta+\omega) \cos (\theta+\omega)=\alpha_{-} \sin (\theta-\omega) \cos (\theta-\omega) .
$$


Then

and

$$
\cos ^{2}(2 \theta)=\frac{\left(\alpha_{+}-\gamma\right)\left(\alpha_{-}-\gamma\right)}{\alpha_{+} \alpha_{-}}
$$

$$
\cos ^{2}(2 \omega)=\frac{\left(\alpha_{+}+\alpha_{-}\right)^{2}\left(\alpha_{+}-\gamma\right)\left(\alpha_{-}-\gamma\right)}{\alpha_{+} \alpha_{-}\left(\alpha_{+}+\alpha_{-}-2 \gamma\right)^{2}}
$$

Assuming that $\alpha_{-} \leq \alpha_{+}$, we note that $\theta$ is real if and only if $\gamma \in\left[0, \alpha_{-}\right] \cup\left[\alpha_{+}, \alpha_{+}+\alpha_{-}\right]$ and $\omega$ is real if and only if either $\alpha_{+}=\alpha_{-}$(and then $\left.\cos ^{2}(2 \omega)=1\right)$ or $\gamma \in\left[0, \alpha_{-}\right] \cup$ $\left[\alpha_{+}, \alpha_{+}+\alpha_{-}\right]$. One can also check that for $\alpha_{+} \neq \alpha_{-}$the cases $\gamma=0, \alpha_{-}, \alpha_{+}, \alpha_{+}+\alpha_{-}$ correspond to $\pi_{00}^{0}, \pi_{01}^{0}, \pi_{10}^{0}$ and $\pi_{11}^{0}$ respectively.

The case $\alpha_{+}=\alpha_{-}:=\alpha$ is slightly different. Here we have: $\cos ^{2}(\theta \pm \omega)=\gamma /(2 \alpha)$, $\cos ^{2}(2 \theta)=(\alpha-\gamma)^{2} / \alpha^{2}$ and $\cos ^{2}(2 \omega)=1$. If $\gamma=\alpha$ then $\zeta_{+}(\theta)$ and $\zeta_{-}(\theta)$ are mutually orthogonal and we may assume that the angle between $\zeta_{ \pm}(\theta)$ and $\xi(\omega)$ is $\pi / 4$.

Lemma 7.8. Under the above choice of parameters, define a function $\psi$ on $S(+,-)$ by putting

$$
\psi(u):=\left[P_{i_{1}} P_{i_{2}} \ldots P_{i_{m}} \xi(\omega), \xi(\bar{\omega})\right]
$$

for $u=i_{1} \ldots i_{m} \in S(+,-)$. Then $\psi$ is the $\left(0,0, \alpha_{+}, \alpha_{-} ; \gamma\right)$-spherical function $\psi_{\gamma}$ on $S(+,-)$. Consequently, the function

$$
\phi(u):=\left[B_{i_{1}} B_{i_{2}} \ldots B_{i_{m}} \xi(\omega), \xi(\bar{\omega})\right],
$$

$B_{ \pm}=\left(1+\tau_{ \pm}\right) P_{ \pm}-\tau_{ \pm} \mathrm{Id}$, is the $\left(\tau_{+}, \tau_{-}, \beta_{+}, \beta_{-} ; \lambda\right)$-spherical function $\phi_{\lambda}$ on $S(+,-)$.

Proof. Assume that the last letter of $u=\epsilon n$ is "-". Then, putting $\nu=\alpha_{+} \delta_{+}+\alpha_{-} \delta_{-}$, we obtain

$$
\begin{aligned}
\left(\psi \diamond_{0} \nu\right)(u) & =\left\langle\psi, \delta_{u} *_{0} \nu\right\rangle=\left\langle\psi, \alpha_{+} \delta_{\epsilon(n+1)}+\alpha_{-} \delta_{\epsilon n}\right\rangle=\alpha_{+} \psi(\epsilon(n+1))+\alpha_{-} \psi(\epsilon n) \\
& =\cos z_{\epsilon} \cos ^{n-1}(2 \theta)\left[\alpha_{+} \cos (2 \theta) \cos (\theta+\omega)+\alpha_{-} \cos (\theta-\omega)\right] .
\end{aligned}
$$

Now we apply (7.7) and (7.5):

$$
\begin{aligned}
& \alpha_{+} \cos (2 \theta) \cos (\theta+\omega)+\alpha_{-} \cos (\theta-\omega) \\
& =\alpha_{+} \cos ^{2}(\theta+\omega) \cos (\theta-\omega)-\alpha_{+} \sin (\theta+\omega) \cos (\theta+\omega) \sin (\theta-\omega)+\alpha_{-} \cos (\theta-\omega) \\
& =\alpha_{+} \cos ^{2}(\theta+\omega) \cos (\theta-\omega)-\alpha_{-} \sin ^{2}(\theta-\omega) \cos (\theta-\omega)+\alpha_{-} \cos (\theta-\omega) \\
& =\left[\alpha_{+} \cos ^{2}(\theta+\omega)+\alpha_{-} \cos ^{2}(\theta-\omega)\right] \cos (\theta-\omega) \\
& =\gamma \cos (\theta-\omega) .
\end{aligned}
$$

so $\left(\psi \diamond_{0} \nu\right)(u)=\gamma \psi(u)$.

Analogous proof works for the other nonzero elements of $S(+,-)$ and for $u=0$ we use $(7.5)$ :

$\left(\psi \diamond_{0} \nu\right)(0)=\alpha_{+} \psi(+)+\alpha_{-} \psi(-)=\alpha_{+} \cos ^{2}(\theta+\omega)+\alpha_{-} \cos ^{2}(\theta-\omega)=\gamma=\gamma \psi(0)$.

If $\beta_{-}\left(1+\tau_{-}\right) \neq \beta_{+}\left(1+\tau_{+}\right)$then for $\lambda=\beta_{+} \tau_{+}-\beta_{-} \tau_{-},-\beta_{+} \tau_{+}+\beta_{-},-\beta_{-} \tau_{-}+\beta_{+}$or $\beta_{+}+\beta_{-}$the function $\phi_{\lambda}$ equals $\widetilde{\phi}_{00}, \widetilde{\phi}_{01}, \widetilde{\phi}_{10}$ or $\widetilde{\phi}_{11}$ respectively. If $\beta_{-}\left(1+\tau_{-}\right)=\beta_{+}\left(1+\tau_{+}\right)$ and $\lambda=-\beta_{+} \tau_{+}+\beta_{-}=-\beta_{-} \tau_{-}+\beta_{+}$, then we have $\phi_{\lambda}=\frac{1}{2}\left(\widetilde{\phi}_{01}+\widetilde{\phi}_{10}\right)$.

Denote $x_{0}:=\left(\beta_{+}\left(1-\tau_{+}\right)+\beta_{-}\left(1-\tau_{-}\right)\right) / 2$,

$$
c_{ \pm}:=\left(\frac{\beta_{+}\left(1+\tau_{+}\right) \pm \beta_{-}\left(1+\tau_{-}\right)}{2}\right)^{2} \text { and } b:=c_{-}+\beta_{+} \beta_{-}\left(\tau_{+}+\tau_{-}\right) .
$$


Proposition 7.9. Let $\phi_{\lambda}$ be the $\left(\tau_{+}, \tau_{-}, \beta_{+}, \beta_{-} ; \lambda\right)$-spherical function and assume that $\beta_{-}\left(1+\tau_{-}\right) \leq \beta_{+}\left(1+\tau_{+}\right)$. Then

i. $\phi_{\lambda}$ is $\left(\tau_{+}, \tau_{-}\right)$-positive definite if and only if $\lambda$ is real and $c_{-} \leq\left(\lambda-x_{0}\right)^{2} \leq c_{+}$or, equivalently,

$$
\lambda \in\left[-\beta_{+} \tau_{+}-\beta_{-} \tau_{-},-\beta_{+} \tau_{+}+\beta_{-}\right] \cup\left[-\beta_{-} \tau_{-}+\beta_{+}, \beta_{+}+\beta_{-}\right] .
$$

ii. $\phi_{\lambda}$ is bounded if and only if either $\tau_{+} \tau_{-}=1$ and

$$
c_{-}-\beta_{+} \beta_{-}\left(1-\tau_{+}\right)\left(1-\tau_{-}\right) \leq\left(\lambda-x_{0}\right)^{2} \leq c_{+}
$$

or $\tau_{+} \tau_{-}<1$ and $\left(\lambda-x_{0}\right)^{2}=x+y$ i satisfies

$$
\left(\frac{x-b}{\beta_{+} \beta_{-}\left(1+\tau_{+} \tau_{-}\right)}\right)^{2}+\left(\frac{y}{\beta_{+} \beta_{-}\left(1-\tau_{+} \tau_{-}\right)}\right)^{2} \leq 1 .
$$

Proof. The condition (7.10) is equivalent to $\gamma \in\left[0, \alpha_{-}\right] \cup\left[\alpha_{+}, \alpha_{+}+\alpha_{-}\right]$(see (7.3)). Then $\theta$ and $\omega$ are real and consequently $\phi_{\lambda}$ is positive definite. On the other hand if a function $\phi$ is $\left(\tau_{+}, \tau_{-}\right)$-positive definite, with $\phi(0)=1$, then $-\tau_{+} \leq \phi(+) \leq 1$ and $-\tau_{-} \leq \phi(-) \leq 1$, which implies (7.10) (see [M1]).

We know that $\pi_{w}^{0}$ is uniformly bounded if and only if $w \in E\left(\tau_{+}, \tau_{-}\right)$. Now we note that

$$
\begin{aligned}
w & =\frac{\left(\alpha_{+}-\gamma\right)\left(\alpha_{-}-\gamma\right)}{\alpha_{+} \alpha_{-}}=\frac{1}{\alpha_{+} \alpha_{-}}\left[\left(\gamma-\frac{\alpha_{+}+\alpha_{-}}{2}\right)^{2}-\left(\frac{\alpha_{+}-\alpha_{-}}{2}\right)^{2}\right] \\
& =\frac{1}{\beta_{+} \beta_{-}\left(1+\tau_{+}\right)\left(1+\tau_{-}\right)}\left[\left(\lambda-x_{0}\right)^{2}-\left(\frac{\beta_{+}\left(1+\tau_{+}\right)-\beta_{-}\left(1+\tau_{-}\right)}{2}\right)^{2}\right],
\end{aligned}
$$

which concludes the proof.

Note that if $\lambda_{1}, \lambda_{2} \in \mathbb{C}$ correspond to $w_{1}, w_{2}$ respectively then $w_{1}=w_{2}$ if and only if either $\lambda_{1}=\lambda_{2}$ or $\lambda_{1}+\lambda_{2}=2 x_{0}$.

We denote by $E\left(\tau_{+}, \tau_{-}, \beta_{+}, \beta_{-}\right)$the closed subset of the complex plane described by (7.11) in the last proposition. Its boundary is an ellipse with the following basic points $(x, 0)$ :

$$
\begin{aligned}
\text { centre: } & x=\left(\frac{\beta_{+}\left(1+\tau_{+}\right)-\beta_{-}\left(1+\tau_{-}\right)}{2}\right)^{2}+\beta_{+} \beta_{-}\left(\tau_{+}+\tau_{-}\right), \\
\text {foci: } & x=\left(\frac{\beta_{+}\left(1+\tau_{+}\right)-\beta_{-}\left(1+\tau_{-}\right)}{2}\right)^{2}+\beta_{+} \beta_{-}\left(\sqrt{\tau_{+}} \pm \sqrt{\tau_{-}}\right)^{2}, \\
\text { left vertex: } & x=\left(\frac{\beta_{+}\left(1+\tau_{+}\right)-\beta_{-}\left(1+\tau_{-}\right)}{2}\right)^{2}-\beta_{+} \beta_{-}\left(1-\tau_{+}\right)\left(1-\tau_{-}\right), \\
\text {right vertex: } & x=\left(\frac{\beta_{+}\left(1+\tau_{+}\right)+\beta_{-}\left(1+\tau_{-}\right)}{2}\right)^{2}
\end{aligned}
$$

Let $F\left(\tau_{+}, \tau_{-}, \beta_{+}, \beta_{-}\right)$denote the set of such $\lambda \in \mathbb{C}$ that $\left(\lambda-x_{0}\right)^{2} \in E\left(\tau_{+}, \tau_{-}, \beta_{+}, \beta_{-}\right)$. We can see that $F\left(\tau_{+}, \tau_{-}, \beta_{+}, \beta_{-}\right)$is connected if $c_{-} \leq \beta_{+} \beta_{-}\left(1-\tau_{+}\right)\left(1-\tau_{-}\right)$, and otherwise $F\left(\tau_{+}, \tau_{-}, \beta_{+}, \beta_{-}\right)$has two components. Note also that if $\tau_{+}, \tau_{-}<1$ and $\beta_{+}\left(1+\tau_{+}\right) \neq \beta_{-}\left(1+\tau_{-}\right)$then there are bounded real spherical functions $\phi_{\lambda}$ which are not $\left(\tau_{+}, \tau_{-}\right)$-positive definite.

Let us take again the free product group $G=G_{+} * G_{-}$, and put $\tau_{ \pm}:=\left(\left|G_{ \pm}\right|-1\right)^{-1}$. If $G_{+}$and $G_{-}$are finite then the condition

$$
\phi \diamond_{\tau}\left(\beta_{+} \delta_{+}+\beta_{-} \delta_{-}\right)=\lambda \cdot \phi
$$

is equivalent to

$$
(\phi \circ t) *\left(\beta_{+} \mu_{+}+\beta_{-} \mu_{-}\right)=\lambda \cdot(\phi \circ t)
$$


(see Proposition 3.1 in [M1]) where

$$
\mu_{ \pm}(x):= \begin{cases}\tau_{ \pm} & \text {if } x \in G_{ \pm} \backslash\{e\} \\ 0 & \text { otherwise. }\end{cases}
$$

Let us now specify our results to the particular case which was investigated by Cartwright and Soardi [CS]. Here we have $\left|G_{+}\right|=r,\left|G_{-}\right|=s, \infty>r>s \geq 2$, $\tau_{+}=1 /(r-1), \tau_{-}=1 /(s-1), \beta_{+}=r-1, \beta_{-}=s-1$, so that $\phi_{\lambda} \circ t$ is an eigenfunction for the convolution with $\chi_{1}$, the characteristic function of elements of length 1 in $G$. Proposition 5.6 and 7.5 yield (cf. Theorem 1 in [CS] and Theorem II in [T2]):

Proposition 7.10. Denote by $\operatorname{sp}\left(\chi_{1}\right)$ and $\operatorname{sp}_{r}\left(\chi_{1}\right)$ the spectrum of $\chi_{1}$ in $C^{*}(G)$ and $C_{r}^{*}(G)$ respectively. Then

$$
\operatorname{sp}\left(\chi_{1}\right)=[-2, s-2] \cup[r-2, r+s-2]
$$

and

$$
\operatorname{sp}_{r}(\chi)=\left[x_{0}-x_{+}, x_{0}-x_{-}\right] \cup\left[x_{0}+x_{-}, x_{0}+x_{+}\right] \cup\{-2, s-2\}
$$

where $x_{0}:=(r+s-4) / 2$ and

$$
x_{ \pm}:=\sqrt{\left(\frac{r-s}{2}\right)^{2}+(\sqrt{r-1} \pm \sqrt{s-1})^{2}} .
$$

Our final proposition is a consequence of Theorem 7.5 (cf. Proposition 8 in [CS]).

Proposition 7.11. Let $\lambda \neq x_{0}:=(r+s-4) / 2$ and assume that $\lambda \notin\{-2, s-2, r-$ $2, r+s-2\}$. Then the spherical function $\phi_{\lambda} \circ t$ on $G=G_{+} * G_{-}$is a coefficient of the representation $\pi_{w}$, where $w=(\lambda+2-r)(\lambda+2-s) /(r s)$. Moreover

i. $\pi_{w}$ is fully irreducible.

ii. If $\left(\lambda-x_{0}\right)^{2}=x+y$ i and

$$
\left(\frac{x-b}{a+1}\right)^{2}+\left(\frac{y}{a-1}\right)^{2}<1,
$$

where $a:=(r-1)(s-1)$ and $b:=\left(\frac{r-s}{2}\right)^{2}+r+s-2$, then $\pi_{w}$ is uniformly bounded. iii. $\pi_{w}$ is unitary if and only if

$$
\lambda \in(-2, s-2) \cup(r-2, r+s-2)
$$

and then $\phi_{\lambda} \circ t$ is positive definite.

The positive definite spherical functions $\phi_{-2} \circ t, \phi_{s-2} \circ t, \phi_{r-2} \circ t$ and $\phi_{r+s-2} \circ t$ are coefficients of the unitary representations $\pi_{00}, \pi_{01}, \pi_{10}$ and $\pi_{11}$, respectively. The representations $\pi_{10}$ and $\pi_{11}$ are irreducible, while $\pi_{00}$ and $\pi_{01}$ are contained in the regular representation of $G$, and thus they are not irreducible.

Comparing formulas (7.10) and (7.13.c) one can see that if $s>2$ then there are bounded real spherical functions $\phi_{\lambda} \circ t$ which are not positive definite.

\section{REFERENCES}

[Av] D. Avitzour, Free product of $C^{*}$-algebras, Trans. Amer. Math. Soc. 271 (1982), 451-477.

[B1] M. Bożejko, Uniformly bounded representation of free groups, J. Reine Angew. Math. 377 (1987), 170-186.

[B2] M. Bożejko, Positive-definite kernels, length functions on groups and noncommutative von Neumann inequality, Studia Math. T. XCV (1989). 
[BS] M. Bożejko, R. Speicher, $\psi$-independent and symmetrized white noises, in Quantum Probability and Related Topics, vol. VI, edited by L. Accardi, World Scientific 1991, 219-236.

[BLS] M. Bożejko, M. Leinert, R. Speicher, Convolution and limit theorems for conditionally free random variables, Pacific J. Math. 175 no. 2 (1996), 357-388.

[CS] D. I. Cartwright, P. M. Soardi, Harmonic analysis on the free product of two cyclic groups, J. Funct. Anal. 65 (2) 1986, 147-171.

[Di] J. Dixmier, $C^{*}$-algebras, North Holland, Amsterdam, New York, Oxford, 1977.

[FP1] A. Figà-Talamanca and M.A. Picardello, Spherical functions and harmonic analysis on free groups, J. Funct. Anal. 47 (1982), 281-304.

[FP2] A. Figà-Talamanca and M.A. Picardello, Harmonic Analysis on Free Groups, Lecture notes in Pure and Applied Mathematics, Dekker, New York, 1983.

[FS] A. Figà-Talamanca and T. Steger, Harmonic analysis for anisotropic random walks on homogeneous trees, Mem. Amer. Math. Soc. 110 (1994), no. 531.

[Ha] U. Haagerup, An example of a non nuclearC ${ }^{*}$-algebra which has the metric approximation property, Inventiones Math, 50 (1979), 280-293.

[Hu] J. E. Humphreys, Reflection groups and Coxeter groups, Cambridge 1990.

[IP] A. Iozzi and M.A. Picardello, Spherical functions on symmetric graphs, in: Harmonic Analysis, Proc. Cortona 1982, Lecture Notes in Math. 992, Springer, 1983, 344-387.

[K] G. Kuhn, Random walks on free products, Ann. Inst. Fourier, Grenoble 41/2 (1991), 467-491.

[KS1] G. Kuhn, T. Steger, A characterisation of the spherical series representations of the free group, Proc. Amer. Math. Soc. 113 (1991), 1085-1096.

[KS2] G. Kuhn, T. Steger, Multiplicative functions on free groups and irreducible representations, Pacific J. Math. 169 (1995), 311-334.

[KS3] G. Kuhn, T. Steger, More irreducible boundary representations, Duke Math. J. 82 (1996), $381-436$.

[KS4] G. Kuhn, T. Steger, Monotony of certain free group representations, J. Funct. Analysis $\mathbf{1 7 9}$ (2001), 1-17.

[MZ] A. M. Mantero, A. Zappa, The Poisson transform on free group and uniformly bounded representations, J. Funct. Anal., 51 (1983), 372-400.

[ML] J. McLaughlin, Random walks and convolution operators on free products, $\mathrm{Ph}$. D. dissertation, 1986 New York University.

[M1] W. Młotkowski, Type-dependent positive definite functions on free product of groups, Colloquium Math. 64 (1993), 41-54.

[M2] W. Młotkowski, Irreducible representations of free products of infinite groups, Colloquium Math. 69, (1995), 193-211.

[M3] W. Młotkowski, Free product of representations, Contemporary Math. 206 (1997), 145-148.

[M4] W. Młotkowski, Free probability on algebras with infinitely many states, Probab. Theory Relat. Fields 115 (1999), 579-596.

[M5] W. Młotkowski, Operator-valued version of the conditionally free product, Studia Math. 153 (2002), 13-30.

[NF] B. Sz.-Nagy and C. Fojas, Harmonic Analysis of Operators on Hilbert Space, North-Holland, 1970.

[P1] W. L. Paschke, Pure eigenstates for the sum of generators of the free group, Pacific J. Math. 197 (2001), 151-171.

[P2] W. L. Paschke, Some irreducible free group representations in which a linear combination of the generators has an eigenvalue, J. Austral. Math. Soc. 72 (2002), 257-286.

[PS] T. Pytlik and R. Szwarc, An analytic family of uniformly bounded representations of free groups, Acta Math. 157 (1986), 286-309.

[Se] J.-P. Serre, Trees, Springer, Berlin 1980.

[Sz] R. Szwarc, Matrix coefficients of irreducible representations of free products of groups, Studia Math. T. XCIV (1989), 179-185.

[T1] A. Trenholme, Maximal abelian subalgebras of function algebras associated with free products, J. Funct. Anal. 79/2, (1988) 342-350.

[T2] A. Trenholme, A Green's function for nonnonhomogeneous random walks on free products, Math. Z 199/3 (1988), 425-441. 
[Vo] D. Voiculescu, Symmetries of some reduced free product $C^{*}$-algebras, in Operator Algebras and their Connection with Topology and Ergodic Theory, Busteni, Romania, 1983, Lecture Notes in Mathematics 1132, Springer Verlag, Heidelberg 1985.

[VDN] D. V. Voiculescu, K. J. Dykema, A. Nica, Free Random Variables, CRM Monograph Series, Volume 1, 1992.

[Wy] J. Wysoczański, An analytic family of uniformly bounded representations of a free product of discrete groups, Pacific J. Math. 157 (1993) 373-387.

Mathematical Institute, University of WrocŁaw, Pl. Grunwaldzki 2/4, 50-384 Wroceaw, POLAND

E-mail address: hebisch@math.uni.wroc.pl, mlotkow@math.uni.wroc.pl 